\title{
PSEUDO-ANOSOV HOMEOMORPHISMS ON TRANSLATION SURFACES IN HYPERELLIPTIC COMPONENTS HAVE LARGE ENTROPY
}

\author{
CORENTIN BOISSY, ERWAN LANNEAU
}

\begin{abstract}
We prove that the dilatation of any pseudo-Anosov homeomorphism on a translation surface that belong to a hyperelliptic component is bounded from below uniformly by $\sqrt{2}$. This is in contrast to Penner's asymptotic. Penner proved that the logarithm of the least dilatation of any pseudoAnosov homeomorphism on a surface of genus $g$ tends to zero at rate $1 / g$ (as $g$ goes to infinity).

We also show that our uniform lower bound $\sqrt{2}$ is sharp. More precisely the least dilatation of a pseudo-Anosov on a genus $g>1$ translation surface in a hyperelliptic component belongs to the interval $\sqrt{2}, \sqrt{2}+2^{1-g}[$. The proof uses the Rauzy-Veech induction.
\end{abstract}

\section{INTRODUCTION}

Pseudo-Anosov homeomorphisms play an important role in Teichmüller theory. Calculating problems for their dilatations have a long history in differential geometry. The unit cotangent bundle of the moduli space of compact genus $g$ Riemann surfaces $\mathcal{M}_{g}$ can be viewed as the moduli space of holomorphic quadratic differentials $Q_{g} \rightarrow \mathcal{M}_{g}$. This space is naturally stratified by strata of quadratic differentials with singularities of prescribed multiplicities. The Teichmüller geodesic flow acts naturally on these strata, and closed loops of length $\log (\theta)>0$ for this flow correspond to conjugacy classes of Pseudo-Anosov homeomorphisms with dilatation $\theta>1$. An important problem concerns the asymptotic behavior of the smallest dilatations.

The strata are not necessarily connected (Kontsevich \& Zorich [KZ03], Lanneau [Lan08]). In this paper we use a discretization of the Teichmüller geodesic flow, i.e. the Rauzy-Veech induction, in order to tackle the minimization problem for hyperelliptic components. This is the first instance of asymptotic for components of the moduli spaces. We shall prove

Theorem. Let $g \geq 2$. Let $\delta_{g}^{\text {hyp }}$ be the least dilatation of pseudo-Anosov homeomorphisms that are affine on translation surfaces belonging to some hyperelliptic connected component of the moduli space $Q_{g}$. Then

$$
\left.\delta_{g}^{h y p} \in\right] \sqrt{2}, \sqrt{2}+\frac{1}{2^{g-1}}[
$$

We will give a more precise statement in the following.

Date: October 24, 2018.

2000 Mathematics Subject Classification. Primary: 37E05. Secondary: 37D40.

Key words and phrases. Pseudo-Anosov homeomorphisms, Interval exchange transformations, Rauzy-Veech induction, Moduli spaces. 
Mapping class group. The mapping class group $\operatorname{Mod}(S)$ of a closed orientable surface $S$ of genus $g \geq 1$ is defined to be the group of homotopy classes of orientation preserving homeomorphisms of $S$. An irreducible mapping class is an isotopy class of homeomorphisms such that no powers preserve a nontrivial subsurface of $S$. By the Thurston-Nielsen classification [Thu88], irreducible mapping classes are either periodic (analogous to roots of unity) or are of a type called pseudo-Anosov [FLP79]. To each pseudo-Anosov mapping class $[\phi] \in \operatorname{Mod}(S)$ one can attach a dilatation factor $\theta(\phi)>1$. The logarithm of $\theta(\phi)$ can be viewed as the minimal topological entropy of any element in the homotopy class of $\phi$ (uniquely realized by some element, $\phi$ ).

Thurston proved that this number is an algebraic integer and even a Perron number. $\theta(\phi)>1$ is also the exponential growth rate of lengths of curves under iteration of $\phi$ (in any metric on $S$ ). These numbers appear naturally as the length spectrum of the moduli space of genus $g$ Riemann surfaces.

It is an open question to characterize the set of dilatations of pseudo-Anosov homeomorphisms. Thurston has conjectured that pseudo-Anosov dilatations (ignoring genus) are precisely the algebraic units that are Perron and also larger than the Galois conjugates of their inverses.

Minimization problem. The set of dilatations for fixed genus is discrete as a subset of $\mathbb{R}$ (see [AY81 Iva88]. Hence the least dilatation $\delta_{g}$ is well defined. We know very little about the values of the constants $\delta_{g}$. The precise value of $\delta_{2}$ has been recently calculated (see Cho \& Ham [CH08] and Lanneau \& Thiffeault [LT10]) but the values of $\delta_{g}$ for $g \geq 3$ are still unknown.

Upper bounds are not hard to derive from examples and there are a lot of results in that direction (see e.g. Penner, McMullen, Hironaka, Kin, Minakawa). So far the best general upper bound for $g \log \left(\delta_{g}\right)$ is the one given by Hironaka [Hir09] and Kin \& Takasawa [KT10]. But again very little is known about lower bounds. Penner [Pen91] proved that $\log \left(\delta_{g}\right) \geq \frac{\log (2)}{6 g-6}$, using general properties of the Perron-Frobenius matrices. There is also a result of Tsai [Tsa09] for pseudo-Anosov on punctured surfaces.

In general lower bounds are much subtle to obtain than upper bounds. In contrast to our understanding of the asymptotic of $\log \left(\delta_{g}\right)$, we still do not know the answer to the following question, posed by McMullen [McM00, Section 10]:

$$
\text { Does } \lim _{g \rightarrow+\infty} g \log \left(\boldsymbol{\delta}_{g}\right) \text { exist? What is its value? }
$$

Subgroups of the modular group and strata. In his book [Far06], Farb proposed two natural refinements of the minimization problem.

The first one is related to subgroups of the modular group. More precisely, let us fix a subgroup $H \subseteq \operatorname{Mod}(S)$ and let us consider the least dilatation $\delta(H)$ of pseudo-Anosov classes $[\phi] \in H$.

In order to state the second problem, let us recall the definition of what we will refer to as strata here (see Section 2 for precise statements). A pseudo-Anosov homeomorphism $\phi$ on a compact surface $S$ defines uniquely a pair of transverse measured foliations on $S$. It is well known [HM79] that these data are equivalent to a pair $(X, q)$ where $X$ is a Riemann surface homeomorphic to $S$ and $q$ is a non zero holomorphic quadratic differential. Moreover $q=\omega^{2}$ (where $\omega$ is an holomorphic 1 -form) if and only if the foliations are orientable; In this case we will call the pair $(X, \omega)$ a translation surface (see Section 2.1). These data endow $X$ with an Euclidean structure for which $\phi$ is affine. 
A stratum is the moduli space of quadratic differentials with prescribed multiplicities of the zeroes of the differentials. Given $\left(k_{1}, \ldots, k_{n}\right)$ satisfying the Gauss-Bonnet equality $\sum_{i=1}^{n} k_{i}=2 g-2$ we define

$$
\delta^{+}\left(k_{1}, \ldots, k_{n}\right):=\inf \left\{\log (\phi): \begin{array}{c}
\text { pseudo-Anosov } \phi \text { whose corresponding }(X, \omega) \\
\text { is in the stratum defined by }\left(k_{1}, \ldots, k_{n}\right)
\end{array}\right\} .
$$

Similarly we define $\delta^{-}\left(k_{1}, \ldots, k_{n}\right)$ for non-orientable measured foliations. These quantities are well defined (up to three exceptions) by a result of Masur \& Smillie [MS93]. Obviously one has

$$
\delta_{g}=\min \left\{\boldsymbol{\delta}^{+}\left(k_{1}, \ldots, k_{n}\right), \boldsymbol{\delta}^{-}\left(k_{1}, \ldots, k_{n}\right)\right\},
$$

where the min is taken over all $k_{i}$ that correspond to genus $g$. It is thus natural to study the minimal dilatation in a given stratum as proposed by Farb [Far06].

It turns out that strata are not connected in general [KZ03, Lan08]. We can thus consider the natural following refinement of Farb's question: give the minimal dilatation of a pseudo-Anosov homeomorphism on a surface in a given connected component of a stratum.

From now on we will restrict to the case where the foliations are orientable. The components are distinguished by two invariants: the parity of the spin structure and the hyperellipticity. The latter are defined as follows.

Recall that a Riemann surface $X$ of genus $g \geq 2$ is hyperelliptic if there exists an holomorphic involution $\tau$ with $2 g+2$ fixed points.

Convention. In all of this paper, we will use the following convention: a translation surface $(X, \omega)$ is hyperelliptic if the underlying Riemann surface is hyperelliptic. The fixed points of $\tau$ are usually called the Weierstrass points.

Definition 1.1. A surface $(X, \omega)$ is in a hyperelliptic connected component if and only if the following hold

(1) $(X, \omega)$ is hyperelliptic,

(2) $\omega$ has at most two zeroes,

(3) $\tau$ permutes the two zeroes (in case of two zeroes).

Remark 1.1. If $(X, \omega)$ is hyperelliptic but does not satisfy the condition (2) or (3) then the property of being hyperelliptic is destroyed by some small perturbations inside the ambient stratum (see [KZ03] for more details).

Leininger [Lei04] and then Farb, Leininger \& Margalit [FLM08] tackle the minimization problem for the subgroups of $\operatorname{Mod}(S)$ given by the Thurston's construction and by the Torelli group, respectively. They provide evidence for the principle that algebraic complexity implies dynamical complexity.

In this paper we will investigate the second problem and prove a similar theorem. This answers a question of Farb [Far06, Problem 7.5]. We shall prove

Theorem 1.1. Let $g \geq 2$. Let $\phi$ be a pseudo-Anosov homeomorphism affine on a genus $g$ translation surface $(X, \omega)$ in a hyperelliptic connected component. Then

$$
\theta(\phi)>\sqrt{2}
$$


Recall that Penner [Pen91] proved that, as the genus increases, there are pseudo-Anosov homeomorphisms with dilatations arbitrarily close to 1 .

This uniform lower bound is sharp as shows Theorem 1.2

Theorem 1.2. Let $g \geq 2$. Let $\delta^{\text {hyp }}(2 g-2)$ (respectively, $\delta^{\text {hyp }}(g-1, g-1)$ ) be the least dilatation of pseudo-Anosov homeomorphisms affine on a genus $g$ translation surface $(X, \omega)$ in the hyperelliptic connected component corresponding to one zero (respectively, two zeroes). Then

$$
\begin{aligned}
& \sqrt{2}<\delta^{\text {hyp }}(2 g-2)<\sqrt{2}+\frac{1}{2^{g-1}}, \text { and } \\
& \sqrt{2}<\delta^{\text {hyp }}(g-1, g-1)<\sqrt{2}+\frac{4}{\sqrt{2}^{g}} .
\end{aligned}
$$

Non hyperelliptic components. The reader may wonder why we impose the restrictions on the action of the hyperelliptic involution on the zeroes in the definition of hyperelliptic connected component. It turns out that if we relax the condition (3) in Definition 1.1 then the asymptotic behavior may be very different if we consider different connected components.

As for example one can construct (see Appendix B) a sequence $\left(\varphi_{g}\right)_{g \geq 3}$ (with $g$ odd) of pseudoAnosov homeomorphisms on a hyperelliptic translation surface $\left(X_{g}, \omega_{g}\right)$ of genus $g$ having two zeroes of degree $g-1, g-1$, and such that the dilatation of $\varphi_{g}$ is the Perron root of the polynomial

$$
X^{2 g}-X^{2 g-1}-4 X^{g}-X+1 .
$$

In particular

$$
\lim _{k \rightarrow+\infty} \theta\left(\varphi_{2 k+1}\right)=1
$$

Of course in that case the hyperelliptic involution fixes the two zeroes. In the above case, the (nonhyperelliptic) components are distinguished by a parity of the spin structure (see [KZ03] and Section 2.2). Let $C_{g}^{\text {odd }}$ be the (non-hyperelliptic) odd component of the stratum with two zeroes in genus $g$. Then we shall prove

Theorem 1.3. Let $g \geq 2$, be an odd integer. Let $\delta\left(C_{g}^{\text {odd }}\right)$ be the least dilatation of pseudo-Anosov homeomorphisms affine on a genus $g$ translation surface $(X, \omega) \in C_{g}^{\text {odd }}$. Then

$$
1<\delta\left(C_{g}^{\text {odd }}\right)<1+\frac{1}{g} .
$$

Quadratic differentials. A quadratic differential $(X, q)$ is strictly quadratic if it is not the square of any holomorphic 1-form. In Section 2.3 we derive from our theorem some results for strict quadratic differentials, namely we will prove Theorem 2.2 , page 8 .

Rauzy-Veech induction. If $C$ is a connected component of some strata then there is a finite ramified covering $\widehat{C} \rightarrow \mathcal{C}$ consisting of marking a zero and a separatrix on surfaces in $\mathcal{C}$. The Rauzy-Veech induction provides a discrete representation (symbolic coding) of the Teichmüller flow on $\widehat{C}$ [Vee82] (see Section 3 for precise definitions). Periodic orbits in $C$ correspond to conjugacy classes of pseudoAnosov on $(X, \omega) \in C$.

As shown by Veech, to each periodic orbit of the Teichmüller geodesic flow $\gamma \subset \widehat{C}$ there corresponds a closed loop in some graph (called a Rauzy diagram) $\mathcal{D}_{r}(C)$ and a renormalization matrix $V(\gamma) \in S L(h, \mathbb{Z})$, where $h=2 g+n-1$. This matrix corresponds to the action of the corresponding 
pseudo-Anosov homeomorphism in relative homology of the underlying surface with respect to the singularities of the Abelian differential. Hence the spectral radius of $V(\gamma)$ is the dilatation of the pseudo-Anosov on $(X, \omega) \in \mathcal{C}$.

Two crucial points in this paper are the following

- we show that "enough" pseudo-Anosov are obtained by the Rauzy-Veech induction;

- we carefully analyze the global geometry of the Rauzy diagrams. This approach was already used by Avila \& Viana [AV07] for the dynamical properties of the Teichmüller geodesic flow.

Outline of a proof of our main result. The Rauzy-Veech induction allows one to relate pseudoAnosov homeomorphisms and closed loops in Rauzy diagrams. We conclude by sketching its use in the proof of Theorem 1.1

1. Let $\phi$ be a pseudo-Anosov homeomorphism affine with respect to a translation surface $(X, \omega)$ in a hyperelliptic component $C^{h y p}$. We prove that $\phi$ commutes with the hyperelliptic involution (Lemma 2.3). Thus $\phi$ induces a pseudo-Anosov homeomorphism on the sphere.

2. Using the Brouwer fixed point theorem, we show that $\phi^{2}$ fixes a separatrix of the horizontal measured foliation on $X$ (Proposition 4.1).

3. Hence $\phi^{2}$ is also affine with respect to a surface $(X, \omega) \in \widehat{C^{h y p}}$ (with a marked separatrix). Thus $\phi^{2}$ is obtained by taking a suitable closed loop $\gamma$ in the Rauzy diagram $\mathcal{D}_{r}^{\text {hyp }}$ corresponding to $\widehat{C^{\text {hyp }} \text {. }}$

4. We use the representation introduced by Kerckhoff [Ker85] and formalized later by Bufetov [Buf06] and Marmi, Moussa \&Yoccoz [MMY05], which furnishes a finite covering $\mathcal{D}^{\text {hyp }} \rightarrow$ $\mathcal{D}_{r}^{\text {hyp }}$. Lift of $\gamma$ in $\mathcal{D}^{\text {hyp}}$, denoted $\hat{\gamma}$, is easier to describe than $\gamma$ in $\mathcal{D}_{r}^{\text {hyp }}$.

5. Analyzing carefully the combinatorics of these Rauzy diagrams we show that the spectral radius of $V(\hat{\gamma})$ is greater than 2 implying $\theta\left(\phi^{2}\right) \geq 2$ (see Section 3.7.2 and Section 4). Since $\theta\left(\phi^{2}\right)=\theta(\phi)^{2}$ one gets the desired result.

6. In Appendix $\mathrm{A}$ we prove that our uniform bound is sharp by exhibiting a suitable sequence of pseudo-Anosov homeomorphisms. In Appendix B we show that the action of the hyperelliptic involution on the zeroes is crucial.

Further results and notes. Fehrenbach \& Los [FL87] proved the following inequality for pseudoAnosov homeomorphisms $\phi$ having a periodic orbit of length $n \geq 3: \log (\phi) \geq \frac{1}{n} \log (1+\sqrt{2})$. However this does not easily imply a uniform lower bound. Indeed for our examples in Appendix A we checked (for $g \leq 10$ ) that

$$
\min \{n \geq 3, \text { there exists a periodic orbit of length } n\}=2 g+1 \text {. }
$$

Our examples also give the systole $\delta^{\text {hyp }}(2 g-2)$ and $\delta^{\text {hyp }}(g-1, g-1)$ for $g \leq 4$ [LT10]. Also we have $\delta_{2}=\delta^{\text {hyp }}(2)=\delta^{-}(1,1,2)$. See also [LT10] for a presentation of the examples in term of the braid group.

Acknowledgements. We thank Vincent Delecroix, Eriko Hironaka, Pascal Hubert, Chris Judge, Chris Leininger, Howard Masur, Curt McMullen, Jean-Luc Thiffeault and Bill Veech, for remarks and comments on this paper. We also thank [Ste09] for computational help. 


\section{BACKGROUND}

We review basic notions and results concerning Abelian differentials, translation surfaces, pseudoAnosov homeomorphisms and moduli spaces. For general references see say [MT02, Vee82, Rau79, MS93, MMY05].

2.1. Flat surfaces and pseudo-Anosov homeomorphisms. A flat surface $S$ is a (real, compact, connected) genus $g$ surface equipped with a flat atlas i.e. a triple $(S, \mathcal{U}, \Sigma)$ such that $\Sigma$ is a finite subset of $S$ (whose elements are called singularities) and $\mathcal{U}=\left\{\left(U_{i}, z_{i}\right)\right\}$ is an atlas of $S \backslash \Sigma$ with transition maps $z \mapsto \pm z+$ constant. We will require that for each $s \in \Sigma$, there is a neighborhood of $s$ isometric to a Euclidean cone. Therefore we get a quadratic differential defined locally in the coordinates $z_{i}$ by the formula $q=d z_{i}^{2}$. This form extends to the points of $\Sigma$ to zeroes, simple poles or marked points (we will usually call the zeroes and poles singular points or simply singularities).

If there exists a sub-atlas such that all transition functions are translations then the quadratic differential $q$ is the global square of an Abelian differential $\omega \in H^{1}(X, \mathbb{C})$. We will then say that $(X, \omega)$ is a translation surface.

A homeomorphism $f: X \rightarrow X$ is an affine homeomorphism if $f$ restricts to a diffeomorphism of $X \backslash \Sigma$ of constant derivative. It is equivalent to say that $f$ restricts to an isomorphism of $X \backslash \Sigma$ which preserves the induced affine structure given by $q$.

Lemma 2.1. If $(X, \omega)$ is a hyperelliptic translation surface then the hyperelliptic involution is affine.

Proof of the lemma. The hyperelliptic involution $\tau$ induces on $H^{1}(X, \mathbb{C})$ a linear involution, which splits this space as a direct sum between an invariant and an anti-invariant subspace. Since the invariant part is isomorphic to $H^{1}\left(\mathbb{P}^{1}(\mathbb{C}), \mathbb{C}\right)$, hence it is trivial. Therefore, $\tau^{*} \omega=-\omega$.

There is a standard classification of elements of $\mathrm{SL}_{2}(\mathbb{R})$ into three types: elliptic, parabolic and hyperbolic. This induces a classification of affine diffeomorphisms. An affine diffeomorphism is parabolic, or elliptic, or pseudo-Anosov, respectively, if $|\operatorname{trace}(D f)|=2,|\operatorname{trace}(D f)|<2$, or $|\operatorname{trace}(D f)|>$ 2 , respectively. If $\phi$ is pseudo-Anosov, in the coordinates of the stable and unstable measured foliations determined by $\phi$, one has $D \phi=\left(\begin{array}{cc}\theta^{-1} & 0 \\ 0 & \theta\end{array}\right)$ where $|\theta|>1$. The number $|\theta|$ is called the dilatation of $\phi$. From now all flat surfaces considered will be translation surfaces, except in Section 2.3 and Appendix B.

For $g \geq 1$, we define the moduli space of Abelian differentials $\mathcal{H}_{g}$ as the moduli space of pairs $(X, \omega)$ where $X$ is a genus $g$ (compact, connected) Riemann surface and $\omega \in \Omega(X)$ a non-zero holomorphic 1 -form defined on $X$. The term moduli space means that we identify the points $(X, \omega)$ and $\left(X^{\prime}, \omega^{\prime}\right)$ if there exists an analytic isomorphism $f: X \rightarrow X^{\prime}$ such that $f^{*} \omega^{\prime}=\omega$. The group $\mathrm{SL}_{2}(\mathbb{R})$ naturally acts on the moduli space of flat surfaces by post composition on the charts.

One can also see a translation surface obtained as a polygon, or a finite union of polygons, whose sides come by pairs, and for each pairs, the corresponding segments are parallel and of equal lengths. These parallel sides are glued together by translation and we assume that this identification preserves the natural orientation of the polygons. In this context, two translation surfaces are identified in the moduli space of Abelian differentials if and only if the corresponding polygons can be obtained from each other by "cutting" and "gluing" and preserving the identifications (i.e. the two surfaces represent 
the same point in the moduli space). Also, the $\mathrm{SL}_{2}(\mathbb{R})$ action in this representation is just the natural linear action on the polygons.

Veech showed that an affine homeomorphism with a derivative map which is not the identity is not isotopic to the identity. Hence a homeomorphism $f$ is an affine homeomorphism on the flat surface $(X, \omega)$, with derivative map $D f=A$, if and only if the matrix $A$ stabilizes the surface $(X, \omega)$. That is $(X, \omega)$ can be obtained from $A \cdot(X, \omega)$ by "cuttings" and "gluings" on the corresponding polygons.

2.2. Connected components of the strata. The moduli space of Abelian differentials is stratified by the combinatorics of the zeroes; We will denote by $\mathcal{H}\left(k_{1}, \ldots, k_{r}\right)$ the stratum of $\mathcal{H}_{g}$ consisting of (classes of) pairs $(X, \omega)$ such that $\omega$ possesses exactly $r$ zeroes on $X$ with multiplicities $\left(k_{1}, \ldots, k_{r}\right)$.

It is a well known part of the Teichmüller theory that these spaces are (Hausdorff) complex analytic, and in fact algebraic, orbifolds.

These strata are non-connected in general but each stratum has at most three connected components (see [KZ03] for a complete classification). In particular for $g \geq 4$ the stratum with a single zero, $\mathcal{H}(2 g-2)$, has three connected components. The stratum $\mathcal{H}(g-1, g-1)$ has two or three connected components depending whether $g$ is even or odd, respectively.

2.2.1. Hyperelliptic component. This component contains precisely pairs $(X, \omega)$ where $X$ is a hyperelliptic surface and $\omega$ is a one-form whose zeroes (if there are two) are interchanged by the hyperelliptic involution. An equivalent formulation is to require that there exists a ramified double cover $\pi: X \rightarrow \mathbb{P}^{1}$ over the sphere and a quadratic differential $q$ on $\mathbb{P}^{1}$ having only one zero and simples poles such that $\omega^{2}=\pi^{*} q$. We will denote these components by $\mathcal{H}^{\text {hyp }}(2 g-2)$ and $\mathcal{H}^{\text {hyp }}(g-1, g-1)$.

2.2.2. Other components. The other (non-hyperelliptic) components are distinguished by a parity of the spin structure. There are two ways to compute the parity of the spin structure of a translation surface $X$. The first way is to use the Arf formula on a symplectic basis (see [KZ03]). The second possibility applies if $X$ comes from a quadratic differential, i.e. if $X$ possesses an involution such that the quotient produces a half-translation surface [Lan04bis]. We will apply this in Appendix B.

2.3. Application of Theorem 1.1 to quadratic differentials. In this section, we extend Theorem 1.1 to some other strata in the moduli space of quadratic differentials. This part is independent from the rest of the paper and can be skipped for a first reading. However, Lemma 2.3 will be needed later.

As for Abelian differentials, strata of the moduli space of quadratic differentials are not connected in general (see [Lan08] for a complete classification). We can deduce from Theorem 1.1 results on some hyperelliptic components in the quadratic case.

We denote by $Q\left(k_{1}, \ldots, k_{n}\right)$ strata of the moduli space of half translation surfaces where the vector $\left(k_{1}, \ldots, k_{n}\right)$ agrees with the Gauss-Bonnet formula $\sum_{i=1}^{n} k_{i}=4 g-4$. For $g \geq 2$, let us consider the two strata $Q(-1,-1,2 g-3,2 g-3)$ and $Q(-1,-1,4 g-2)$. Their hyperelliptic component can be defined as follows (see [Lan04]):

$$
\begin{gathered}
C_{1}^{\text {hyp }}:=\left\{(X, q): \begin{array}{l}
X \text { is hyperelliptic, } \tau \text { is affine for } q,(X, q) \in Q(-1,-1,2 g-3,2 g-3), \\
\text { and the involution permutes the two zeroes and the two poles }
\end{array}\right\} . \\
C_{2}^{\text {hyp }}:=\left\{(X, q): \begin{array}{l}
X \text { is hyperelliptic, } \tau \text { is affine for } q,(X, q) \in Q(-1,-1,4 g-2), \\
\text { and the involution permutes the two poles }
\end{array}\right\} .
\end{gathered}
$$


Theorem 2.2. Let $g \geq 2$. Let $C$ be $C_{1}^{\text {hyp }}$ or $C_{2}^{\text {hyp }}$ defined above. Let $\phi$ be a pseudo-Anosov homeomorphism affine on a half translation surface $(X, q) \in \mathcal{C}$. Then the dilatation $\theta(\phi)$ satisfies $\theta(\phi)>\sqrt{2}$.

We will use the following result.

Lemma 2.3. Let $f$ be an affine homeomorphism on hyperelliptic flat surface of genus greater than or equal to two. The map $f$ commutes with the hyperelliptic involution $\tau$.

Proof of Lemma 2.3 Since the derivative map $D f$ of $f$ is constant in the affine charts, the conjugate $f^{-1} \tau f$ is conformal and is an involution with $2 g+2$ fixed points. It is thus the hyperelliptic involution $\tau$ [FK92]. This proves the result.

Proof of Theorem 2.2 Let $(X, q) \in Q(-1,-1,2 g-3,2 g-3)$ be a half translation surface in the hyperelliptic component. Let $\phi$ be a pseudo-Anosov homeomorphism on $X$ and $\tau$ the hyperelliptic involution.

Passing to the quotient we get a meromorphic quadratic differential $q^{\prime}$ on the projective line $\mathbb{P}^{1}$ with $2 g+1$ simple poles and a single zero of degree $2 g-3$. Taking the standard orientating cover $\pi: Y \rightarrow \mathbb{P}^{1}$ over $\mathbb{P}^{1}$ having ramification points precisely over odd degree singularities (namely the poles and the zero) we obtain a translation surface $(Y, \omega)$ where $\omega^{2}=\pi^{*} q^{\prime}$. By construction (see Subsection 2.2.1) $(Y, \omega)$ belongs to the hyperelliptic component $\mathcal{H}^{\text {hyp }}(2 g-2)$. Now by Lemma $2.3 \phi$ commutes with $\tau$ on $Y$. Thus $\phi$ induces a pseudo-Anosov homeomorphism $\varphi$ on $\mathbb{P}^{1}$ with the same dilatation. Since $\varphi$ preserves the set of ramification points of $\pi, \varphi$ lifts to a new pseudo-Anosov homeomorphism, say $\hat{\varphi}$, on $Y$.

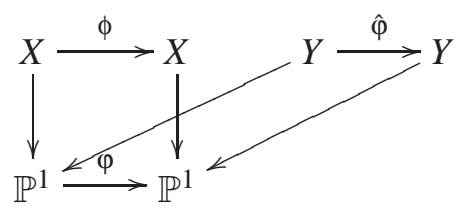

Now $\theta(\phi)=\theta(\varphi)=\theta(\hat{\varphi})$. By Theorem 1.1 we get that $\theta(\phi)>\sqrt{2}$.

The second case is similar and left to the reader.

\section{RAUZY-VEECH INDUCTION AND PSEUDO-ANOSOV HOMEOMORPHISMS}

In this section we recall the basic construction of pseudo-Anosov homeomorphisms using the Rauzy-Veech induction (for details see [Vee82], §8, and [Rau79, MMY05]). We first review the link between interval exchange maps and translation surfaces.

3.1. Interval exchange transformations. Let $I \subset \mathbb{R}$ be an open interval and let us choose a finite partition of $I$ into $d \geq 2$ open subintervals $\left\{I_{j}, j=1, \ldots, d\right\}$. An interval exchange transformation is a one-to-one map $T$ from $I$ to itself that permutes, by translation, the subintervals $I_{j}$. It is easy to see that $T$ is precisely determined by the following data: a permutation that encodes how the intervals are exchanged, and a vector with positive entries that encodes the lengths of the intervals.

We use the representation introduced first by Kerckhoff [Ker85] and formalized later by Bufetov [Buf06] and Marmi, Moussa \&Yoccoz [MMY05]. This will simplify the description of the induction which will be very useful for the proof of our result. 
We will attribute a name to each interval $I_{j}$. In this case, we will speak of labeled interval exchange maps. One gets a pair of one-to-one maps $\left(\pi_{t}, \pi_{b}\right)$ (t for "top" and b for "bottom") from a finite alphabet $\mathcal{A}$ to $\{1, \ldots, d\}$ in the following way. In the partition of $I$ into intervals, we denote the $k^{\text {th }}$ interval, when counted from the left to the right, by $I_{\pi_{t}^{-1}(k)}$. Once the intervals are exchanged, the interval number $k$ is $I_{\pi_{b}^{-1}(k)}$. Then with this convention, the permutation encoding the map $T$ is $\pi_{b} \circ \pi_{t}^{-1}$. We will denote the length of the intervals by a vector $\lambda=\left(\lambda_{\alpha}\right)_{\alpha \in \mathfrak{A}}$.

Definition 3.1. We will call the pair $\left(\pi_{t}, \pi_{b}\right)$ a labeled permutation, and $\pi_{b} \circ \pi_{t}^{-1}$ a permutation (or reduced permutation). If it is clear from the context, then we will just use the term permutation. We will also usually write a reduced permutation as a labeled one with $\mathcal{A}=\{1, \ldots, d\}$ and $\pi_{t}=I d$.

One usually represents labeled permutations $\pi=\left(\pi_{t}, \pi_{b}\right)$ by a table:

$$
\pi=\left(\begin{array}{llll}
\pi_{t}^{-1}(1) & \pi_{t}^{-1}(2) & \ldots & \pi_{t}^{-1}(d) \\
\pi_{b}^{-1}(1) & \pi_{b}^{-1}(2) & \ldots & \pi_{b}^{-1}(d)
\end{array}\right)
$$

3.2. Suspension data. The next construction provides a link between interval exchange transformations and translation surfaces. A suspension datum for $T=(\pi, \lambda)$ is a collection of vectors $\left\{\tau_{\alpha}\right\}_{\alpha \in \mathcal{A}}$ such that

- $\forall 1 \leq k \leq d-1, \sum_{\pi_{t}(\alpha) \leq k} \tau_{\alpha}>0$,

- $\forall 1 \leq k \leq d-1, \sum_{\pi_{b}(\alpha) \leq k} \tau_{\alpha}<0$.

We will often use the notation $\zeta=(\lambda, \tau)$. To each suspension datum $\tau$, we can associate a translation surface $(X, \omega)=X(\pi, \zeta)$ in the following way.

Consider the broken line $L_{t}$ on $\mathbb{C}=\mathbb{R}^{2}$ defined by concatenation of the vectors $\zeta_{\pi_{t}^{-1}(j)}$ (in this order) for $j=$ $1, \ldots, d$ with starting point at the origin. Similarly, we consider the broken line $L_{b}$ defined by concatenation of the vectors $\zeta_{\pi_{b}^{-1}(j)}$ (in this order) for $j=1, \ldots, d$ with starting point at the origin. If the lines $L_{t}$ and $L_{b}$ have no intersections

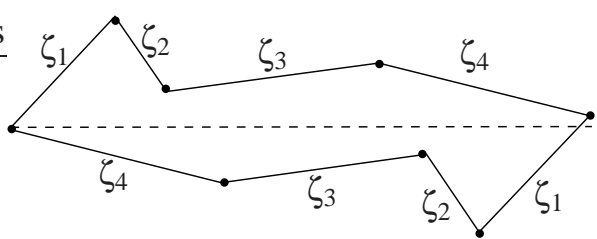
other than the endpoints, we can construct a translation surface $X$ by identifying each side $\zeta_{j}$ on $L_{t}$ with the side $\zeta_{j}$ on $L_{b}$ by a translation. The resulting surface is a translation surface endowed with the form $d z^{2}$. Note that the lines $L_{t}$ and $L_{b}$ might have some other intersection points. But in this case, one can still define a translation surface by using the zippered rectangle construction, due to Veech ([Vee82]).

Let $I \subset X$ be the horizontal interval defined by $I=\left(0, \sum_{\alpha} \lambda_{\alpha}\right) \times\{0\}$. Then the interval exchange transformation $T$ is precisely the one defined by the first return map of the vertical flow on $X$ to $I$.

Each $(\pi, \lambda)$ with $\pi$ irreducible (i.e. $\pi_{t}^{-1}(\{1, \ldots, k\}) \neq \pi_{b}^{-1}(\{1, \ldots, k\})$ for all $1 \leq k<d$ ) admits a suspension data.

3.3. Rauzy-Veech induction. The Rauzy-Veech induction $\mathcal{R}(T)$ of $T$ is defined as the first return map of $T$ to a certain subinterval $J$ of $I$ (see [Rau79, MMY05] for details).

We recall very briefly the construction. Following [AGY06] we define the type of $T$ by $t$ if $\lambda_{\pi_{t}^{-1}(d)}>$ $\lambda_{\pi_{b}^{-1}(d)}$ and $b$ if $\lambda_{\pi_{t}^{-1}(d)}<\lambda_{\pi_{b}^{-1}(d)}$. When $T$ is of type $t$ (respectively, $b$ ) we will say that the label $\pi_{t}^{-1}(d)$ 
(respectively, $\pi_{b}^{-1}(d)$ ) is the winner and that $\pi_{b}^{-1}(d)$ (respectively, $\pi_{t}^{-1}(d)$ ) is the looser. We define a subinterval $J$ of $I$ by

$$
J= \begin{cases}I \backslash T\left(I_{\pi_{b}^{-1}(d)}\right) & \text { if } T \text { is of type t; } \\ I \backslash I_{\pi_{t}^{-1}(d)} & \text { if } T \text { is of type b. }\end{cases}
$$

The image of $T$ by the Rauzy-Veech induction $\mathcal{R}$ is defined as the first return map of $T$ to the subinterval $J$. This is again an interval exchange transformation, defined on $d$ letters (see e.g. [Rau79]). The data of $\mathcal{R}(T)$ are very easy to express in term of those of $T$.

There are two cases to distinguish depending whether $T$ is of type $t$ or $b$; the labeled permutations of $\mathcal{R}(T)$ only depends on $\pi$ and on the type of $T$. If $\varepsilon \in\{t, b\}$ is the type of $T$, this defines two maps $\mathcal{R}_{t}$ and $\mathcal{R}_{b}$ by $\mathcal{R}(T)=\left(\mathcal{R}_{\varepsilon}(\pi), \lambda^{\prime}\right)$. We will often make use of the following notation: if $\varepsilon \in\{t, b\}$ we denote by $1-\varepsilon$ the other element of $\{t, b\}$.

(1) $T$ has type $t$. Let $k \in\{1, \ldots, d-1\}$ such that $\pi_{b}^{-1}(k)=\pi_{t}^{-1}(d)$. Then $\mathcal{R}_{t}\left(\pi_{t}, \pi_{b}\right)=\left(\pi_{t}^{\prime}, \pi_{b}^{\prime}\right)$ where $\pi_{t}=\pi_{t}^{\prime}$ and

$$
\pi_{b}^{\prime-1}(j)= \begin{cases}\pi_{b}^{-1}(j) & \text { if } j \leq k \\ \pi_{b}^{-1}(d) & \text { if } j=k+1 \\ \pi_{b}^{-1}(j-1) & \text { otherwise. }\end{cases}
$$

(2) $T$ has type $b$. Let $k \in\{1, \ldots, d-1\}$ such that $\pi_{t}^{-1}(k)=\pi_{b}^{-1}(d)$. Then $\mathcal{R}_{b}\left(\pi_{t}, \pi_{b}\right)=\left(\pi_{t}^{\prime}, \pi_{b}^{\prime}\right)$ where $\pi_{b}=\pi_{b}^{\prime}$ and

$$
\pi_{t}^{\prime-1}(j)= \begin{cases}\pi_{t}^{-1}(j) & \text { if } j \leq k \\ \pi_{t}^{-1}(d) & \text { if } j=k+1 \\ \pi_{t}^{-1}(j-1) & \text { otherwise. }\end{cases}
$$

(3) Let us denote by $E_{\alpha \beta}$ the $d \times d$ matrix of which the $\alpha, \beta$-th element is equal to 1 , all others to 0 . If $T$ is of type $t$ then let $(\alpha, \beta)=\left(\pi_{t}^{-1}(d), \pi_{b}^{-1}(d)\right)$ otherwise let $(\alpha, \beta)=\left(\pi_{b}^{-1}(d), \pi_{t}^{-1}(d)\right)$. Then $V_{\alpha \beta} \lambda^{\prime}=\lambda$, where $V_{\alpha \beta}$ is the transvection matrix $I+E_{\alpha \beta}$.

Remark 3.1. In the Veech's original construction, the matrices used to obtain $\lambda^{\prime}$ in terms of $\lambda$ were more complicated: of the form $P+E_{\alpha \beta}$ where $P$ is a permutation matrix. Indeed, after the Rauzy induction "bottom", we usually have $\pi_{t}^{\prime} \neq I d$, and we must "renumber" the intervals.

This construction is due to Rauzy. This induction is called the Rauzy-Veech induction since Veech observed that one can actually define the induction on the suspension data in the following way. If $\tau$ is a suspension data over $(\pi, \lambda)$ then we define $\mathcal{R}(\pi, \lambda, \tau)$ by

$$
\mathcal{R}(\pi, \lambda, \tau)=\left(\mathcal{R}_{\varepsilon}(\pi), V^{-1} \lambda, V^{-1} \tau\right),
$$

where $\varepsilon$ is the type of $T=(\pi, \lambda)$ and $V$ is the corresponding transition matrix. In other terms $V_{\alpha \beta} \zeta^{\prime}=\zeta$ where $\zeta=(\lambda, \tau)$.

Remark 3.2. By construction the two translation surfaces $X(\pi, \zeta)$ and $X\left(\pi^{\prime}, \zeta^{\prime}\right)$ are naturally isometric (as translation surfaces). 
Now if we iterate the Rauzy induction, we get a sequence $\left(\alpha_{k}, \beta_{k}\right)$ of winners/losers. Denoting $\mathcal{R}^{(n)}(\pi, \lambda)=\left(\pi^{(n)}, \lambda^{(n)}\right)$, the transition matrix that rely $\lambda^{(n)}$ to $\lambda$ is the product of the transition matrices:

$$
\left(\prod_{k=1}^{n} V_{\alpha_{k} \beta_{k}}\right) \lambda^{(n)}=\lambda .
$$

\subsection{Rauzy diagrams.}

3.4.1. Labeled Rauzy diagrams. For a labeled permutation $\pi$, we call the labeled Rauzy diagram, denoted by $\mathcal{D}(\pi)$, the graph whose vertices are all labeled permutations that we can obtained from $\pi$ by the combinatorial Rauzy moves. From each vertices, there are two edges labeled $t$ and $b$ (the type) corresponding to the two combinatorial Rauzy moves. We will denote by $\pi \stackrel{\alpha, \beta}{\longrightarrow} \pi^{\prime}$ for the edge corresponding to $\mathscr{R}_{\varepsilon}(\pi)=\pi^{\prime}$ where $\varepsilon \in\{t, b\}$ and $\alpha / \beta$ is the winner/looser. To each path $\gamma$ is this diagram, there is thus a sequence of winners/losers. We will denote by $V(\gamma)$ the product of the transition matrices in Equation (1). The next lemma is clear from the definition.

Lemma 3.1. Let $\gamma_{n}=\pi_{1} \ldots \pi_{n}$ be a path in the labeled Rauzy diagram, and let $V_{n}$ be the matrix associated to the path $\gamma_{n}$. Let $\alpha, \beta$ be the winner/looser associated to the edge $\pi_{n-1} \stackrel{\alpha, \beta}{\longrightarrow} \pi_{n}$. Then $V_{n}$ is obtained from $V_{n-1}$ by adding the column $\alpha$ to the column $\beta$.

Definition 3.2. A closed path in the labeled Rauzy diagram is said to be primitive if the associated matrix $V$ is primitive, i.e. if there exists a power of $V$ such that all the entries are positive. We will also say that a path contains the letter $\alpha$ as winner (respectively, looser) if it contains the edge $\stackrel{\alpha, \beta}{\longrightarrow}$. (respectively, $\stackrel{\beta, \alpha}{\longrightarrow} \cdot$ ), for some $\beta$.

We have the following proposition (see [MMY05], proposition in section 1.2.3),

Proposition 3.2 (Marmi, Moussa \& Yoccoz). A closed path $\gamma$ in a labeled Rauzy diagram is primitive if and only if $\gamma$ contains all the letters as winner at least once.

3.4.2. Reduced Rauzy diagrams. We have previously defined Rauzy induction and Rauzy diagrams for labeled interval exchange transformations. One can also define the same for reduced interval exchange transformations, as it was first, for which the corresponding labeled permutation is just a permutation of $\{1, \ldots, d\}$ (see [Vee82]). These are obtained after identifying $\left(\pi_{t}, \pi_{b}\right)$ with $\left(\pi_{t}^{\prime}, \pi_{b}^{\prime}\right)$ if $\pi_{b} \circ \pi_{t}^{-1}=\pi_{b}^{\prime} \circ \pi_{t}^{\prime-1}$. In the next we will use the notation $\mathcal{D}_{r}(\pi)$ to denote the reduced Rauzy diagram associated to the permutation $\pi$.

Note that the labeled Rauzy diagram is naturally a covering of the reduced Rauzy diagram, and they are usually not isomorphic.

Convention. Let $\pi$ be a permutation. We will denote by $\mathcal{D}(\pi)$ the labeled Rauzy diagram of $\pi$ and by $\mathcal{D}_{r}(\pi)$ the reduced one.

Given a closed path $\gamma$ in the reduced Rauzy diagram, as previously, one can associate a matrix $V$ as follow: we take $\left(\pi_{t}, \pi_{b}\right)$ the labeled permutation corresponding to the endpoint of $\gamma$ so that $\pi_{t}=I d$. Then we consider $\hat{\gamma}$ a lift of $\gamma$ in the labeled Rauzy diagram. The path $\hat{\gamma}$ is not necessarily closed and it ends at a permutation $\left(\pi_{t}^{\prime}, \pi_{b}^{\prime}\right)$. We can associate to it a matrix $\widehat{V}$ as before. Let $P$ be the permutation 
matrix defined by permuting the columns of the $d \times d$ identity matrix according to the permutation $\pi_{t}^{\prime}$, i.e. the $P=\left[p_{i j}\right]$, with $p_{i j}=1$ if $j=\pi_{t}^{\prime}(i)$ and 0 otherwise. The transition matrix associated to the path is then:

$$
V=\widehat{V} \cdot P
$$

As before, a closed path in the reduced Rauzy diagram is called primitive if $V$ is primitive. A standard reference for the next two sections is [Vee82].

3.5. Construction of pseudo-Anosov homeomorphisms. There is a natural $\mathrm{SL}_{2}(\mathbb{R})$-action on the strata. In particular, the one-parameter subgroup $g_{t}=\left(\begin{array}{cc}e^{t} & 0 \\ 0 & e^{-t}\end{array}\right)$ is called the Teichmüller geodesic flow. It can be shown that conjugacy classes of pseudo-Anosov homeomorphisms are one-to-one with closed geodesics of the Teichmüller geodesic flow on strata. There is a very nice construction of pseudo-Anosov homeomorphisms using the Rauzy-Veech induction, and we recall now this construction.

Let $\pi$ be an irreducible permutation and let $\gamma$ be a closed loop in the reduced Rauzy diagram associated to $\pi$. One can associate to $\gamma$ a matrix $V(\gamma)$ (see section above). Let us assume that $V$ is primitive and let $\theta>1$ be its Perron-Frobenius eigenvalue. We choose a positive eigenvector $\lambda$ for $\theta$. It can be shown that $V$ is symplectic [Vee82], thus let us choose an eigenvector $\tau$ for the eigenvalue $\theta^{-1}$ with $\tau_{\pi_{0}^{-1}(d)}>0$. It turns out that $\tau$ defines a suspension data over $T=(\pi, \lambda)$. Indeed, the set of suspension data is an open cone, that is preserved by $V^{-1}$. Since the matrix $V^{-1}$ has a dominant eigenvalue $\theta$ (for the eigenvector $\tau$ ), the vector $\tau$ must belong to this cone. If $\zeta=(\lambda, \tau)$, one has

$$
\begin{aligned}
\mathcal{R}(\pi, \zeta)=\left(\pi, V^{-1} \zeta\right)=\left(\pi, V^{-1} \lambda, V^{-1} \tau\right)=\left(\pi, \theta^{-1} \lambda, \theta \tau\right) & \\
& =g_{t}(\pi, \lambda, \tau), \quad \text { where } \quad t=\log (\theta)>0 .
\end{aligned}
$$

Hence the two surfaces $X(\pi, \zeta)$ and $g_{t} X(\pi, \zeta)$ differ by some element of the mapping class group (see Remark 3.2). In other words there exists a pseudo-Anosov homeomorphism $\phi$ affine with respect to the translation surface $X(\pi, \zeta)$ and such that $D \phi=g_{t}$. The action of $\phi$ on the relative homology of $(X, \omega)$ is $V(\gamma)$ thus the dilatation of $\phi$ is $\theta$. Note that by construction $\phi$ fixes the zero on the left of the interval $I$ and also a horizontal separatrix adjacent to this zero (namely, the oriented half line corresponding to the interval $I$ ).

It turns out that this construction is very general as we will see in the coming section.

3.6. Discrete representation of the geodesic flow. Let us fix an irreducible permutation $\pi$ defined over $d$ letters. If $C$ is the connected component of some stratum, let $\widehat{C}$ be the ramified cover over $C$ obtained by considering the set of triplets $(X, \omega, l)$ where $(X, \omega) \in C$ and $l$ is a horizontal separatrix adjacent to a zero of $\omega$.

Clearly the set of $(\lambda, \tau)$ such that $\tau$ is a suspension data over $\pi$ is a connected space and the map $(\lambda, \tau)=\zeta \mapsto X(\pi, \zeta)$ is continuous. Thus all surfaces obtained by this construction belong to the same connected component of some strata, say $\mathcal{C}(\pi) \subset \mathcal{H}(\sigma)$. Moreover $\sigma$ can be computed easily in terms of $\pi$. We also define $\widehat{C}(\pi)$ to be the set of $(X(\pi, \zeta), l)$ where $l$ is the horizontal separatrix that corresponds to $\mathbb{R}^{+} \times\{0\}$ in the Veech's construction. We define

$$
\mathcal{T}(\widehat{C})=\{(\pi, \zeta) ; \widehat{C}(\pi)=\widehat{C}, \text { and } \zeta \text { is a suspension datum for } \pi\} .
$$


The Rauzy-Veech induction is (almost everywhere) well defined and one-to-one on $\mathcal{T}(\widehat{\mathcal{C}})$. Hence let $\mathcal{H}(\widehat{\mathcal{C}})$ be the quotient of $\mathcal{T}(\widehat{C})$ by the induction.

The Veech zippered rectangle's construction provides (almost everywhere) a one-to-one map $Z$ : $\mathcal{H}(\widehat{C}) \rightarrow \widehat{C}$ (see [Boi09] for details).

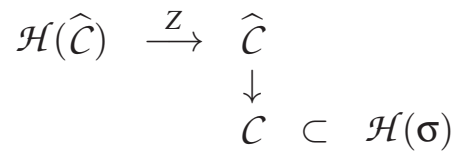

One can define the Teichmüller geodesic flow on $\mathcal{H}(\widehat{\mathcal{C}})$ by $g_{t}(\pi, \zeta)=\left(\pi, g_{t} \zeta\right)$. The Teichmüller flow on $C$ lifts to a flow $g_{t}$ on $\widehat{C}$. It is easy to check that $g_{t}$ is equivariant with $Z$ i.e. $g_{t} Z=Z g_{t}$.

By construction, periodic orbits of $g_{t}$ on $\mathcal{C}$ corresponding to pseudo-Anosov homeomorphisms that fix the separatrix $I$ lift to periodic orbits on $\widehat{C}$ for $g_{t}$. Thus they produce periodic orbits for $g_{t}$ acting on the level of suspensions $\mathcal{H}(\widehat{C})$.

In fact Veech proved that all pseudo-Anosov homeomorphisms fixing a separatrix arise in this way. To see that one can show that the subset of $\mathcal{T}(\widehat{\mathcal{C}})$ defined by

$$
\left\{(\pi, \zeta) \in \mathcal{T}(\widehat{C}) ; 1 \leq|\operatorname{Re}(\zeta)| \leq 1+\min \left(\operatorname{Re}\left(\zeta_{\pi_{0}^{-1}(d)}\right), \operatorname{Re}\left(\zeta_{\pi_{1}^{-1}(d)}\right)\right)\right\}
$$

is a fundamental domain of $\mathcal{T}(\widehat{\mathcal{C}})$ for the quotient map $\mathcal{T}(\widehat{\mathcal{C}}) \rightarrow \mathcal{H}(\widehat{\mathcal{C}})$, and the Poincare map of the Teichmüller flow on the section

$$
\mathcal{S}=\{(\pi, \zeta) ; \pi \text { irreducible, }|\operatorname{Re}(\zeta)|=1\} / \sim
$$

is precisely the renormalized Rauzy-Veech induction on suspensions:

$$
\widehat{\mathcal{R}}(\pi, \lambda):=\left(\mathcal{R}_{\varepsilon}(\pi), V^{-1} \lambda /\left|V^{-1} \lambda\right|\right) .
$$

We can summarize the above discussion by the following theorem.

Theorem (Veech). Let $\gamma$ be a closed loop, based at $\pi$, in a reduced Rauzy diagram $\mathcal{D}_{r}(\pi)$ and let $V=V(\gamma)$ be the product of the associated transition matrices. Let us assume that $V$ is primitive. Let $\lambda$ be a positive eigenvector for the Perron-Frobenius eigenvalue $\theta$ of $V$ and let $\tau$ be an eigenvector for the eigenvalue $\theta^{-1}$ of $V$ with $\tau_{\pi_{0}^{-1}(1)}>0$. Then

(1) $\zeta=(\lambda, \tau)$ is a suspension datum for $T=(\pi, \lambda)$;

(2) The matrix $A=\left(\begin{array}{cc}\theta^{-1} & 0 \\ 0 & \theta\end{array}\right)$ is the derivative map of an affine pseudo-Anosov homeomorphism $\phi$ on $X(\pi, \zeta)$; The action on relative homology of $\phi$ is given by the matrix $V$ (in a suitable basis).

(3) The dilatation of $\phi$ is $\theta$;

(4) All pseudo-Anosov homeomorphisms that fix a separatrix are obtained in this way.

We will use this theorem in order to prove our main result.

\subsection{Examples of labeled and reduced Rauzy diagrams.}




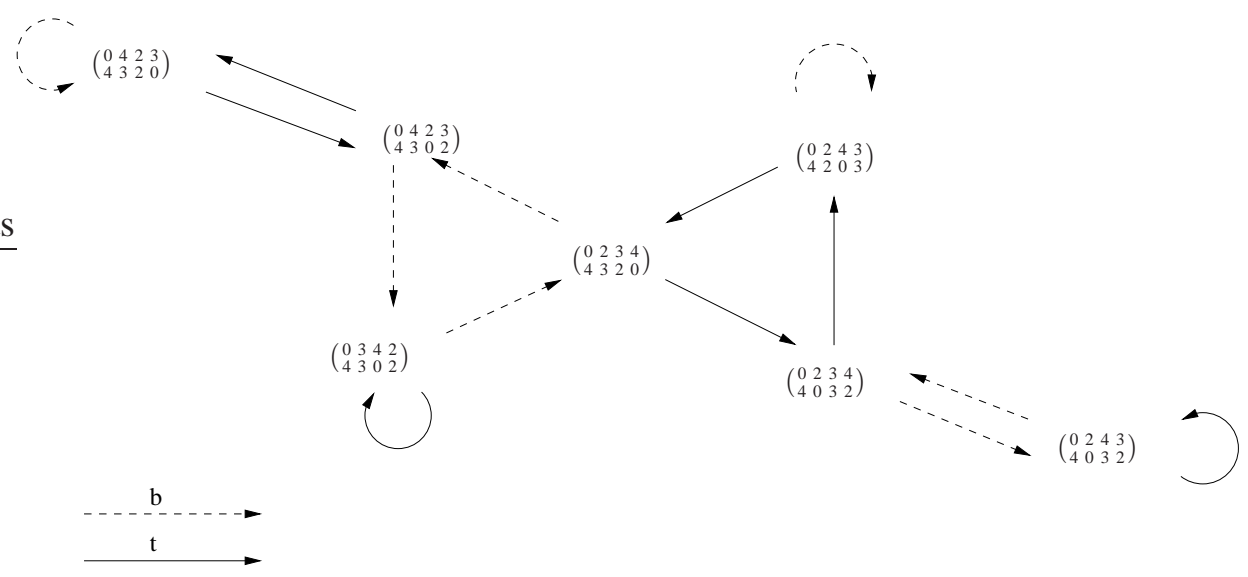

FIGURE 1. The diagram $\mathcal{D}^{h y p}=\mathcal{D}\left(\tau_{n}\right) \approx \mathcal{D}_{r}\left(\tau_{n}\right)$ for $n=4$.

3.7.1. Hyperelliptic connected components. Let $n \geq 2$. A representative labeled permutation for the connected component $\mathcal{H}^{\text {hyp }}(2 g-2)$ (respectively, $\mathcal{H}^{\text {hyp }}(g-1, g-1)$ ) is

$$
\tau_{n}=\left(\begin{array}{cccccc}
0 & 2 & 3 & \ldots & n-1 & n \\
n & n-1 & \ldots & 3 & 2 & 0
\end{array}\right)
$$

where $n=2 g$ (respectively, $n=2 g+1$ ). We use these "non standards" labels in order to simplify the notations in the next sections. It turns out that these labeled and reduced Rauzy diagrams are isomorphic. The precise description of the diagrams was given by Rauzy [Rau79]. Let us recall his result here.

If $\pi=\left(\pi_{t}, \pi_{b}\right)$ is a labeled permutation, for $\varepsilon \in\{t, b\}$ we define $G^{\varepsilon}(\pi)$ to be the subdiagram of the Rauzy diagram of $\pi$ whose vertices are obtained from $\pi$ by a simple path, and whose first step is the map $\mathcal{R}_{\varepsilon}$. Recall that for $\varepsilon \in\{t, b\}$, we denote by $1-\varepsilon$ the other element of $\{t, b\}$.

Proposition (Rauzy). Let $\tau_{n}$ be the labeled permutation defined in Equation (3). Then

(1) The vertices of the Rauzy diagram of $\tau_{n}$ is the disjoint union of the vertices of $G^{0}\left(\tau_{n}\right), G^{1}\left(\tau_{n}\right)$, and $\left\{\tau_{n}\right\}$.

(2) Let $\tau_{n, k}=\mathcal{R}_{\varepsilon}^{k}\left(\tau_{n}\right)$, for some $\varepsilon \in\{t, b\}$ and $k \in\{1, \ldots, n-1\}$. Then $G^{1-\varepsilon}\left(\tau_{n, k}\right)$ is naturally isomorphic to $G^{1-\varepsilon}\left(\tau_{n-k}\right)$.

(3) The cardinality of the Rauzy diagram is $2^{n-1}-1$.

Using this result, one can show that the labeled and reduced diagrams are isomorphic. We will denote by $\mathcal{D}^{\text {hyp }}=\mathcal{D}\left(\tau_{n}\right) \approx \mathcal{D}_{r}\left(\tau_{n}\right)$ this Rauzy diagram. A consequence of the previous proposition is the following:

Corollary 3.3. Let $\gamma$ be a closed (oriented) path in $\mathcal{D}^{\text {hyp }}$. Assume that it contains the step $\pi \stackrel{\alpha, \beta}{\longrightarrow} \pi^{\prime}$ with $\pi \neq \pi^{\prime}$, then there exists $\beta^{\prime}$ such that it contains the step $\pi^{\prime} \stackrel{\alpha, \beta^{\prime}}{\longrightarrow} \pi^{\prime \prime}$.

Proof. A consequence of the Rauzy description of $\mathcal{D}^{\text {hyp }}$ is that $\mathcal{D}^{\text {hyp }} \backslash\left\{\pi^{\prime}\right\}$ is not connected any more. Assume that, in the step following $\pi \stackrel{\alpha, \beta}{\longrightarrow} \pi^{\prime}$, the symbol $\alpha$ is looser, then the new permutation and $\pi$ belong to two different connected components of $\mathcal{D}^{h y p} \backslash \pi^{\prime}$. Looking at the sequence of permutations 
that appear, one must come back to the connected component that contains $\pi$, and hence, the step $\pi^{\prime} \stackrel{\alpha, \beta^{\prime}}{\longrightarrow} \pi^{\prime \prime}$ eventually appears.

3.7.2. Hyperelliptic connected components with a marked point. Here we present a second family of Rauzy diagrams that will be useful for our proof. There description is a little bit more technical, but will be needed later. We start with a very informal description of the labeled Rauzy diagram. The precise description can be skipped in a first reading.

The labeled Rauzy diagram is a covering of the reduced one. The cardinalities of these labeled Rauzy diagrams have been calculated (see Delecroix [Del10]). The degree of the covering is also known [Boi10].

A fundamental domain of this covering can be roughly seen as a copy of the hyperelliptic Rauzy diagram described previously, with some added permutations (see Figure 2). This fundamental domain (a leaf) is composed at first glance by two principal loops that intersect in a central permutation, and on the over vertices of these loops starts a secondary loop. The whole diagram is obtained by taking several copies of this "leaf". The different leaves are joined together by the transition permutations: each secondary loops contains a unique such transition permutation, and a "k-th secondary loop" of a leaf is attached to a "k-th secondary loop" of another leaf (see Figure 3 , and compare it with Figure 2).

We now give the precise description of this diagram. A representative permutation for the connected component $\mathcal{H}^{\text {hyp }}(0,2 g-2)$ (respectively, $\mathcal{H}^{\text {hyp }}(0, g-1, g-1)$ ), with a regular marked point, is

$$
\pi_{n}=\left(\begin{array}{ccccccc}
0 & 2 & 3 & \ldots & n-1 & 1 & n \\
n & n-1 & \ldots & 3 & 2 & 1 & 0
\end{array}\right),
$$

where $n=2 g$ (respectively, $n=2 g+1$ ). Contrary to the previous case, the labeled diagram and the reduced diagram are not isomorphic anymore. As we will see the cardinality of the reduced diagram is $2^{n-1}-1+n$ and the cardinality of the labeled diagram is $\left(2^{n-1}-1+n\right)(n-1)$.

Our next goal is to describe the reduced Rauzy diagrams $\mathcal{D}\left(\pi_{n}\right)$ and $\mathcal{D}_{r}\left(\pi_{n}\right)$ associated to the above permutation $\pi_{n}$. The key point is to observe that if we forbid the letter 1 to be winner or looser in the construction of the diagram $\mathcal{D}\left(\pi_{n}\right)$ starting from $\pi$, then one gets more or less the diagram $\mathcal{D}^{h y p}$ (compare with Avila \& Viana [AV07]). So we can obtain a subdiagram of $\mathcal{D}\left(\pi_{n}\right)$ starting from $\mathcal{D}^{\text {hyp }}$ as follows.

(1) To each permutation $\pi \in \mathcal{D}^{\text {hyp }}$ construct a new permutation $\tilde{\pi} \in \mathcal{D}\left(\pi_{n}\right)$ by adding the letter 1 to the left of the letter $n$ on the top and to the left of the letter 0 on the bottom. We will refer to the augmented hyperelliptic diagram.

(2) Replace each edge $\pi \stackrel{k, n}{\longrightarrow} \pi^{\prime}$ in $\mathcal{D}^{h y p}$ by two edges $\tilde{\pi} \stackrel{k, n}{\longrightarrow} \pi^{\prime \prime} \stackrel{k, 1}{\longrightarrow} \tilde{\pi}^{\prime}$ in $\mathcal{D}\left(\pi_{n}\right)$, where the permutation $\pi^{\prime \prime}$ has the letter 1 for the last letter on the top.

(3) Replace each edge $\pi \stackrel{k, 0}{\longrightarrow} \pi^{\prime}$ in $\mathcal{D}^{h y p}$ by two edges $\tilde{\pi} \stackrel{k, 0}{\longrightarrow} \pi^{\prime \prime} \stackrel{k, 1}{\longrightarrow} \tilde{\pi}^{\prime}$ in $\mathcal{D}\left(\pi_{n}\right)$, where the permutation $\pi^{\prime \prime}$ has the letter 1 for the last letter on the bottom.

(4) We will denote by $\mathcal{A}_{n} \subset \mathcal{D}\left(\pi_{n}\right)$ the added permutations of the operations (2) and (3). All the edges are built except the cases when $\pi^{\prime \prime} \in \mathcal{A}_{n}$ and where 1 is winner.

Observe that operation (2) (respectively, (3)) arises exactly when the letter $n$ (respectively, 0) is looser in the diagram $\mathcal{D}^{h y p}$. Hence the description given in the previous section of this hyperelliptic diagram leads to the following. 


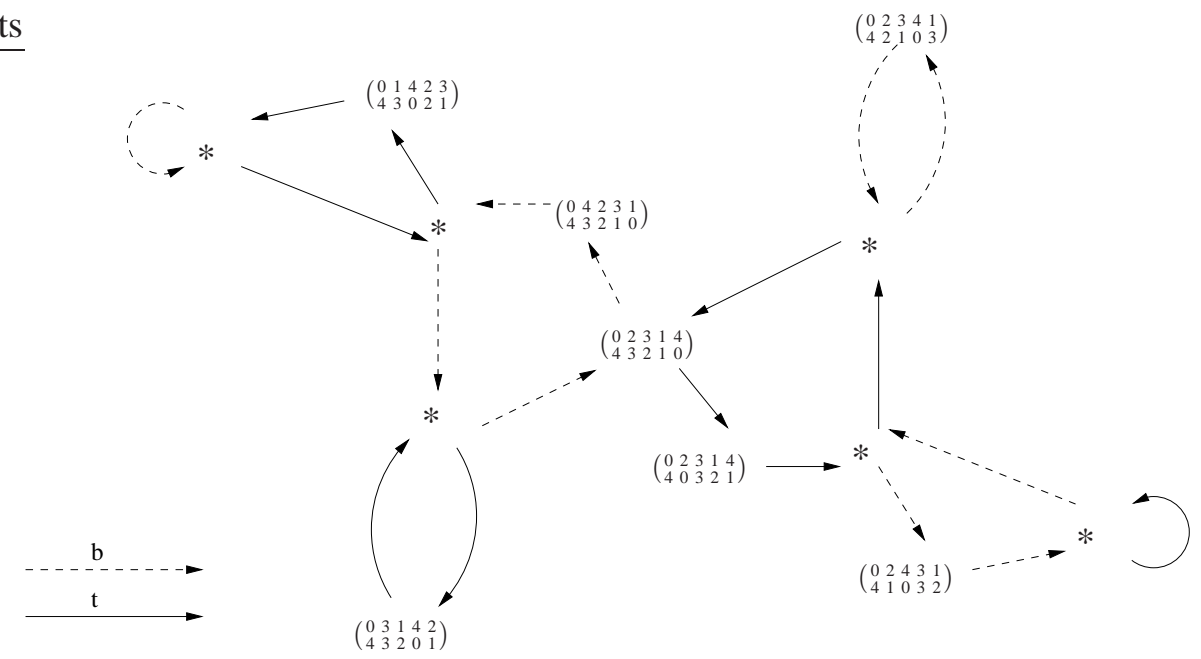

FIGURE 2. The augmented hyperelliptic diagram, and added permutations for $n=4$.

Lemma 3.4. In $\mathcal{D}^{\text {hyp }}$, the edges where $n$ (respectively, 0) is looser are

$$
\left.\mathcal{R}_{t}^{k}\left(\tau_{n}\right) \stackrel{*, n}{\longrightarrow} \mathcal{R}_{b} \mathcal{R}_{t}^{k}\left(\tau_{n}\right) \quad \text { (respectively, } \mathcal{R}_{b}^{k}\left(\tau_{n}\right) \stackrel{*, 0}{\longrightarrow} \mathcal{R}_{t} \mathcal{R}_{b}^{k}\left(\tau_{n}\right)\right)
$$

for $k \in\{0, \ldots, n-2\}$. So, there are $n-1$ edges where $n$ is looser and $n-1$ edges where 0 is looser.

Therefore, the added permutations $\mathcal{A}_{n}$ in $\mathcal{D}\left(\pi_{n}\right)$ by the operations (2) and (3) are:

$$
\begin{aligned}
\mathcal{R}_{b} \mathcal{R}_{t}^{k}\left(\pi_{n}\right) & =\left(\begin{array}{cccccccccc}
0 & 2 & \ldots & k-1 & k & n & k+1 & \ldots & n-1 & 1 \\
n & k-1 & \ldots & 2 & 1 & 0 & n-1 & \ldots & k+1 & k
\end{array}\right), \\
\mathcal{R}_{t} \mathcal{R}_{b}^{k}\left(\pi_{n}\right) & =\left(\begin{array}{cccccccc}
0 & n-k+2 & \ldots & n-1 & 1 & n & 2 & \ldots \\
n & n-1 & \ldots & n-k+2 & n-k+1 & 0 & n-k & \ldots
\end{array}\right]
\end{aligned}
$$

for $k \in\{2, \ldots, n-1\}$, and

$$
\begin{aligned}
& \mathcal{R}_{t}\left(\pi_{n}\right)=\left(\begin{array}{cccccccc}
0 & 2 & 3 & \ldots & n-1 & 1 & n \\
n & 0 & n-1 & \ldots & 3 & 2 & 1
\end{array}\right) \\
& \mathcal{R}_{b}\left(\pi_{n}\right)=\left(\begin{array}{ccccccc}
0 & n & 2 & 3 & \ldots & n-1 & 1 \\
n & n-1 & \ldots & 3 & 2 & 1 & 0
\end{array}\right)
\end{aligned}
$$

In addition for all $k=2, \ldots, n-1$ the two new permutations in the labeled diagram $\mathcal{D}\left(\pi_{n}\right)$ defined by (5) and (6) correspond to the same permutation in the reduced diagram $\mathcal{D}_{r}\left(\pi_{n}\right)$; the renumbering corresponds to $\sigma^{-k}$ with $\sigma$ the cyclic permutation $(1,2, \ldots, n-1)$. The two new permutations corresponding to $\mathcal{R}_{t}\left(\pi_{n}\right)$ and $\mathcal{R}_{b}\left(\pi_{n}\right)$ are different in $\mathcal{D}_{r}\left(\pi_{n}\right)$.

Proof of Lemma 3.4 The proof is obtained by straightforward computation and is left to the reader.

Now in order to finish the construction of $\mathcal{D}\left(\pi_{n}\right)$ and $\mathcal{D}_{r}\left(\pi_{n}\right)$, one has to consider from the added permutations $\mathcal{A}_{n}$ the operations top/bottom where the letter 1 is winner. In fact it turns out that the reduced diagram $\mathcal{D}_{r}\left(\pi_{n}\right)$ is already constructed. Namely,

Corollary 3.5. The diagram $\mathcal{D}_{r}\left(\pi_{n}\right)$ corresponds to adding the new permutations defined in above Lemma 3.4 namely $\mathcal{T}_{n}$ to the augmented hyperelliptic diagram, up to renumbering. In particular the cardinality of this diagram is $2^{n-1}-1+n$. 
Proof of Corollary 3.5 Let $\pi^{\prime} \in \mathcal{A}_{n}$ be an added permutation. Assume that it is given by (5), that is $\pi^{\prime}=\mathcal{R}_{b} \mathcal{R}_{t}^{k}\left(\pi_{n}\right)$ for some $k$. The edge $\pi^{\prime} \rightarrow \mathcal{R}_{b}\left(\pi^{\prime}\right)$ was constructed when defining $\mathcal{A}_{n}$. By Lemma 3.4 we also have that $\pi^{\prime}=\mathcal{R}_{\mathcal{L}} \mathcal{R}_{b}^{k}\left(\pi_{n}\right)$ in the reduced Rauzy diagram, hence the edge $\pi^{\prime} \rightarrow \mathcal{R}_{t}\left(\pi^{\prime}\right)$ was also already constructed. This corresponds to $n-2$ added permutations.

The remaining cases are when $\pi^{\prime}$ is given by (7) or (8). Then it is clear that the array corresponding to 1 being winner are arrays from $\pi^{\prime}$ to itself. This corresponds to 2 permutations.

Hence, the diagram $\mathcal{D}_{r}\left(\pi_{n}\right)$ is completely built. Since $\# \mathcal{D}^{h y p}=2^{n-1}-1$ one has $\# \mathcal{D}_{r}\left(\pi_{n}\right)=2^{n-1}-$ $1+n$.

Lemma 3.4 and Corollary 3.5 imply the following description of the diagram $\mathcal{D}\left(\pi_{n}\right)$.

Proposition 3.6. The augmented hyperelliptic diagram together with the permutations $\mathcal{T}_{n}$ form a fundamental domain for the covering map $\mathcal{D}\left(\pi_{n}\right) \longrightarrow \mathcal{D}_{r}\left(\pi_{n}\right)$. A fiber for this covering consists of labeled permutations of the kind $\left\{\pi, \pi \circ \sigma, \ldots, \pi \circ \sigma^{n-2}\right\}$, where $\pi \circ \sigma^{k}$ means the labeled permutation $\pi$ after renumbering with $\sigma^{k}$. In particular the cardinality of this diagram is $\left(2^{n}-1+n\right)(n-1)$.

The following technical lemma is similar to Corollary 3.3 It is a very important lemma since it will allow us to give information about all irreducible paths.

Lemma 3.7. Let $\gamma$ be a closed oriented path in $\mathcal{D}\left(\pi_{n}\right)$ : Let $k \in\{2, \ldots, n-2\}$ and let $k^{\prime} \in\{2, n-k\}$ and let $\varepsilon \in\{t, b\}$.

a) If $\gamma$ contains the step $\mathcal{R}_{\varepsilon}^{k^{\prime}-1} \mathcal{R}_{1-\varepsilon}^{k}\left(\pi_{n}\right) \rightarrow \mathcal{R}_{\varepsilon}^{k^{\prime}} \mathcal{R}_{1-\varepsilon}^{k}\left(\pi_{n}\right)$, then it also contains the following step $\mathcal{R}_{\varepsilon}^{k^{\prime}} \mathcal{R}_{1-\varepsilon}^{k}\left(\pi_{n}\right) \rightarrow \mathcal{R}_{\varepsilon}^{k^{\prime}+1} \mathcal{R}_{1-\varepsilon}^{k}\left(\pi_{n}\right)$.

b) If $\gamma$ contains the step $\mathcal{R}_{\varepsilon}^{k-1}\left(\pi_{n}\right) \rightarrow \mathcal{R}_{\varepsilon}^{k}\left(\pi_{n}\right)$, then it also contains the step $\mathcal{R}_{\varepsilon}^{k}\left(\pi_{n}\right) \rightarrow \mathcal{R}_{\varepsilon}^{k+1}\left(\pi_{n}\right)$, or the step $\mathcal{R}_{1-\varepsilon}^{k}\left(\pi_{n} \circ \sigma^{i}\right) \rightarrow \mathcal{R}_{1-\varepsilon}^{k+1}\left(\pi_{n} \circ \sigma^{i}\right)$, for some $i \in\{0, \ldots, n-2\}$.

This property is also true if $\gamma$ is a nonclosed path that starts and ends in the set $\left\{\pi_{n}, \pi_{n} \circ \sigma, \ldots, \pi_{n} \circ\right.$ $\left.\sigma^{n-2}\right\}$.

Proof. a) Using the construction of $\mathcal{D}\left(\pi_{n}\right)$ from $\mathcal{D}^{\text {hyp }}$, we see that, as in Corollary 3.3, the diagram $\mathcal{D}\left(\pi_{n}\right) \backslash\left\{\mathcal{R}_{\varepsilon}^{k^{\prime}} \mathcal{R}_{1-\varepsilon}^{k}\left(\pi_{n}\right)\right\}$ is nonconnected.

b) Here, we have that $\mathcal{D}\left(\pi_{n}\right) \backslash\left\{\mathcal{R}_{\varepsilon}^{k}\left(\pi_{n}\right), \mathcal{R}_{1-\varepsilon}^{k}\left(\pi_{n} \circ \sigma^{i}\right)\right\}$ is nonconnected, for the parameter $i$ such that $\mathcal{R}_{1-\varepsilon} \mathcal{R}_{\varepsilon}^{k}\left(\pi_{n}\right)=\mathcal{R}_{\varepsilon} \mathcal{R}_{1-\varepsilon}^{k}\left(\pi_{n} \circ \sigma^{i}\right)$.

\section{Minimal Dilatations in A HYPERELLiPTiC CONNECTED COMPONENT}

4.1. A key proposition. Veech's construction can only build a pseudo-Anosov homeomorphism fixing a separatrix. But there can be many pseudo-Anosov homeomorphism that do not fix any separatrix [Los09, Lan10]. In our case, we are saved by the following key proposition.

Proposition 4.1. Let $g \geq 1$ and let $(X, \omega)$ be a flat surface in some hyperelliptic connected component $\mathcal{H}^{\text {hyp }}(2 g-2)$ or $\mathcal{H}^{\text {hyp }}(g-1, g-1)$. Then for any pseudo-Anosov homeomorphism $\phi$ affine with respect to $(X, \omega), \phi^{2}$ has a fixed point of negative index i.e. $\phi^{2}$ fixes a point and a outgoing separatrix issued from that point.

Proof of Proposition 4.1 Let $\tau$ be the hyperelliptic involution on $X$. Firstly by Lemma 2.3 , the involution $\tau$ commutes with $\phi$. Thus $\phi$ descends to a pseudo-Anosov homeomorphism $f$ on the sphere. 


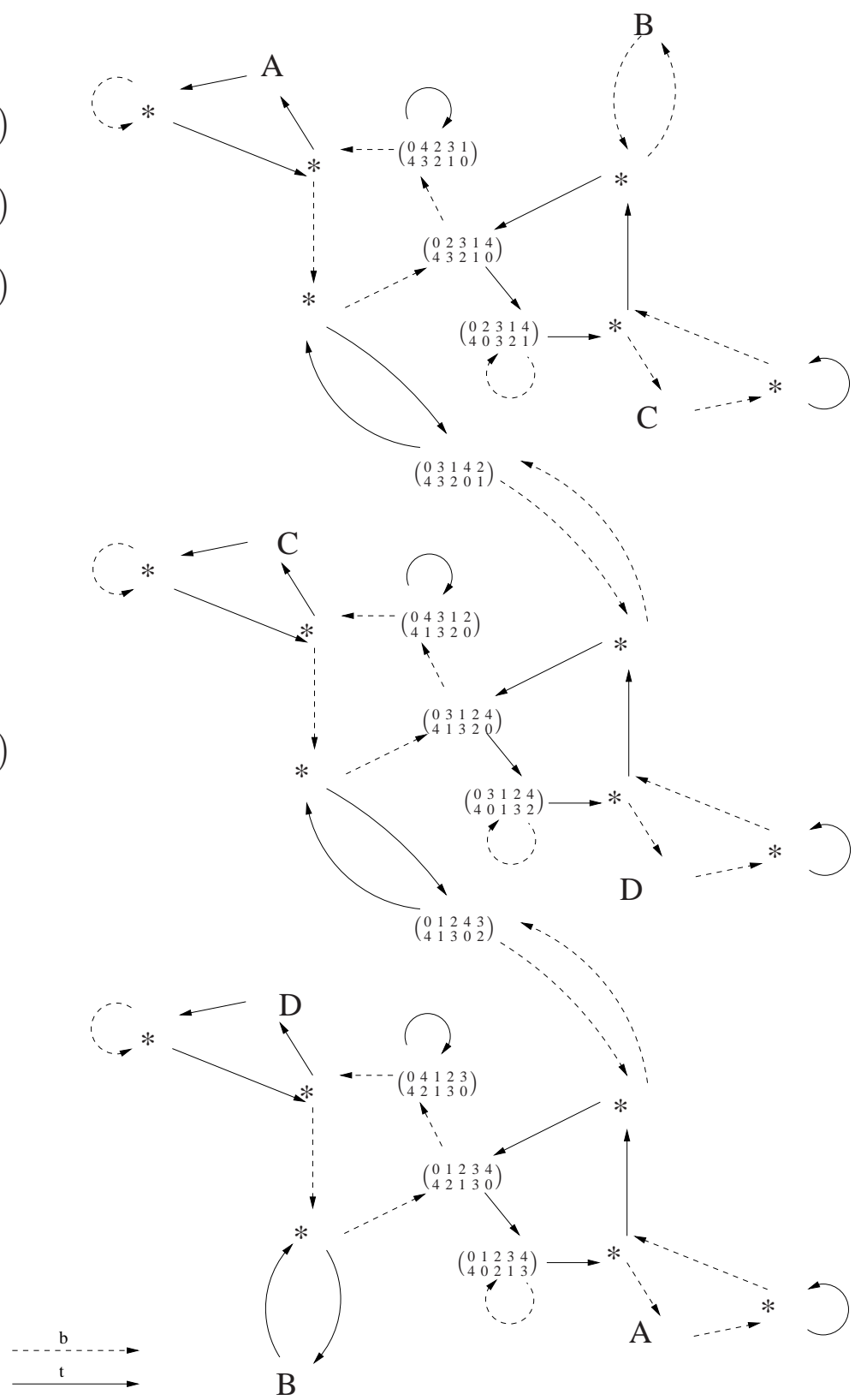

FIGURE 3 . The complete diagram $\mathcal{D}\left(\pi_{n}\right)$ for $n=4$. The vertices labeled by letters $A, B, C$ and $D$ should be identified.

By assumption, the quadratic differential associated to $f$ has a single zero (of order $2 g-3$ or $2 g-2$ depending the case). Thus $f$ fixes this zero and induces a disc pseudo-Anosov homeomorphism, say $g$. Remark that $g$ can be defined as a homeomorphism on the closed disc by extending it to the rays 
emanating from the zero. Now by Brouwer's theorem, $g$ has a fixed point on the disc. Either this point is inside the disc (thus this is a regular point) or on the boundary. If the last case occur, then $f$ fixes the separatrices issued from the singularity. It is then not hard to see that either $\phi^{2}$ fixes a regular point and the outgoing separatrix, or a singular point and the outgoing separatrices. Proposition 4.1 is proven.

Remark 4.1. Another Brouwer's theorem states that a homeomorphism on the plane with a periodic orbit has a fixed point. With this theorem, it is easy to show that there actually exists a regular fixed point for $\phi^{2}$. However, we believe that the case where the separatrix is attached to a singularity is useful to present the main ideas without the technical difficulties of the other case.

The next two sections analyze the two cases depending whether the fixed point is singular or regular.

4.2. Case when the fixed separatrix is adjacent to the singularity. In this section, we study the case when the pseudo-Anosov homeomorphism fixes a horizontal separatrix starting from a singular point. We thus have to consider the corresponding diagrams $\mathcal{D}^{\text {hyp }}$. We first prove a sufficient condition for a primitive path to have a transition matrix with a spectral radius greater than 2 .

Proposition 4.2. Let $\gamma$ be a primitive closed path in some reduced Rauzy diagram. Let us assume that there is a lift $\hat{\gamma}$ of $\gamma$ in the labeled Rauzy diagram that contains all the letters as losers, or all the letters as winners. Then the Perron-Frobenius eigenvalue of the matrix $V(\gamma)$ is bounded from below by 2.

Proof of the proposition. If $\hat{\gamma}$ contains all the looser (respectively, all the winner) then Lemma 3.1 implies that the minimum of the sums of the columns (respectively, the lines) of the corresponding matrix $\widehat{V}$, before remuneration, is at least 2. Since $V(\gamma)=\widehat{V} \cdot P$ where $P$ is a permutation matrix (see Equation 2) the same property on the sums of the columns (respectively, the lines) holds for $V(\gamma)$.

By assumption, the matrix $V=V(\gamma)$ is primitive. Up to replacing $V$ by ${ }^{t} V$, which does not change its eigenvalues, we can assume that the sum of the coefficients on each lines is at least 2. Let $x$ be a Perron-Frobenius eigenvector with positive entries associated to the Perron-Frobenius eigenvalue $\rho(V)$ of $V$. Let $i_{0}$ be such that $\min _{j=1, \ldots, d} x_{j}=x_{i_{0}}>0$. Then

$$
\rho(V) x_{i_{0}}=\sum_{j=1}^{d} v_{i_{0} j} x_{j} \geq x_{i_{0}} \sum_{j=1}^{d} v_{i_{0} j} .
$$

Since $x_{i_{0}}>0$, we see that there exists $i_{0}$ such that

$$
\rho(V) \geq \sum_{j=1}^{d} v_{i_{0} j} \geq 2 .
$$

Proposition 4.3. Let $\phi$ be a pseudo-Anosov homeomorphism affine on a translation surface $(X, \omega) \in$ $\mathcal{H}^{\text {hyp }}(2 g-2)$ or $(X, \omega) \in \mathcal{H}^{\text {hyp }}(g-1, g-1)$. If $\phi$ fixes a horizontal separatrix emanating from a singular point of $\omega$ then the dilatation of $\phi$ is bounded from below by 2 .

Proof. By Veech's Theorem, $\phi$ is obtained by taking a closed loop in a Rauzy diagram. By a Lemma of Rauzy (see [Rau79]), any Rauzy diagram contains a vertex of the kind:

$$
v=\left(\begin{array}{lll}
\alpha & \ldots & \beta \\
\beta & \ldots & \alpha
\end{array}\right)
$$


Since the underlying flat surface is in the hyperelliptic connected component, it is easy to see that $v=\tau_{n}$ and therefore this Rauzy diagram is necessarily $\mathcal{D}^{h y p}$ (one can also use the main theorem of [Boi09]). Has we have seen, the labeled and reduced diagrams coincide. Hence, for any closed path in the reduced diagram whose associated matrix is primitive, the corresponding lift contains all the letters as winner (see Proposition 3.2). Hence by Proposition 4.2 the associated dilatation is at least 2.

4.3. Case when the fixed separatrix is adjacent to a regular point. One needs to prove the following proposition.

Proposition 4.4. Let $\phi$ be a pseudo-Anosov homeomorphism on a surface in either $\mathcal{H}^{\text {hyp }}(2 g-2)$ or $\mathcal{H}^{\text {hyp }}(g-1, g-1)$. If $\phi$ fixes a separatrix adjacent to a regular point, then the dilatation of $\phi$ is bounded from below by 2 .

The idea of the proof is similar to the one of Proposition 4.3. we must consider pseudo-Anosov homeomorphisms that are obtained by the Veech construction using the diagram $\mathcal{D}_{r}\left(\pi_{n}\right)$, were $\pi_{n}=$ $\left(\begin{array}{ccccccc}0 & 2 & 3 & \ldots & n-1 & 1 & n \\ n & n-1 & \ldots & 3 & 2 & 1 & 0\end{array}\right)$. In this case, the reduced diagram is different from the labeled diagram (see section 3.7.2). But we will show that we can still apply Proposition 4.2

Remark 4.2. Our case is more subtle than the previous case. Indeed, start with the permutation

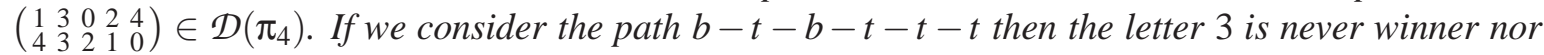
looser. But the corresponding path in $\mathcal{D}_{r}\left(\pi_{4}\right)$ is closed and primitive. Nevertheless the lift based at the permutation $\left(\begin{array}{lllll}1 & 2 & 3 & 0 & 4 \\ 4 & 3 & 2 & 0 & 1\end{array}\right)$ is $t-t-t-b-t-b$, and all the letters are looser.

We will call the permutations in the diagram $\mathcal{D}\left(\pi_{n}\right)$ that are in $\left\{\pi_{n}, \pi_{n} \circ \sigma, \ldots, \pi_{n} \circ \sigma^{n-2}\right\}$ the central permutations. We will call permutations in the diagram $\mathcal{D}\left(\pi_{n}\right)$ that corresponds to $\mathcal{A}_{n}$ up to renumbering with $\sigma^{k}$ the transition permutations.

We have the following technical lemmas.

Lemma 4.5. Let $\gamma$ be a primitive closed path in $\mathcal{D}_{r}\left(\pi_{n}\right)$. There is a lift $\hat{\gamma}$ of $\gamma$ in $\mathcal{D}\left(\pi_{n}\right)$, not necessarily closed, that starts and ends at central permutations.

Lemma 4.6. Let $\hat{\gamma}$ be a path in $\mathcal{D}_{r}\left(\pi_{n}\right)$ that starts and ends at central permutations. Then $\hat{\gamma}$ contains all the letters $0,1,2, \ldots, n$ as looser.

We first prove the proposition assuming Lemma 4.5 and Lemma4.6

Proof of Proposition 4.4 Let $\phi$ be a pseudo-Anosov homeomorphism that fixes a separatrix adjacent to a regular point. By Veech's theorem $\phi$ is obtain by taking a closed loop in some Rauzy diagram. Using Rauzy Lemma stated in the proof of Proposition 4.3. we can show that the Rauzy diagram is necessarily $\mathcal{D}_{r}\left(\pi_{n}\right)$ (one can also use the main theorem of [Boi09]). Let $\gamma$ be the corresponding closed primitive path in $\mathcal{D}_{r}\left(\pi_{n}\right)$. By Lemma 4.5, there is a lift of $\gamma$ that starts and ends at central permutations. This lift contains all the letters as losers by Lemma 4.6, and by Proposition 4.2, the Perron-Frobenius eigenvalue of the corresponding matrix, and hence the dilatation of $\phi$ is at least 2 .

The proof of the lemmas is strongly related to the geometry of the diagram $\mathcal{D}\left(\pi_{n}\right)$. Before giving a formal proof, we first present a very informal proof that uses the informal description of the diagram given in the beginning of Section 3.7.2. 
(1) For the first lemma it is enough to prove that a primitive closed path in the labeled Rauzy diagram must pass through a central permutation. Such path must pass through a principal loop were 0 or $\mathrm{n}$ is winner. If it enters in a $\mathrm{k}$-th secondary loop (attached to the $\mathrm{k}$-th vertex of a principal loop), the geometry of the diagram imposes that it either leaves the secondary loop from the same vertex (and therefore joins the $(\mathrm{k}+1)$-th vertex of the principal loop) or joins a $\mathrm{k}$-th secondary loop in another leaf and escape it at the k-th vertex of another principal loop, and therefore joins the $(\mathrm{k}+1)$-th vertex of this loop. Iterating this argument, it eventually joins a central permutation.

(2) The key observation for the second lemma is that a path joining two central permutations will either pass through all the vertices of a principal loop, or will pass from one principal loop to another one through a transition permutation and the corresponding pair of secondary loops. In any cases all the letters will appear as losers.

We now give a proof of the lemmas.

Proof of Lemma 4.5 Let $\gamma$ be a primitive closed path in $\mathcal{D}_{r}\left(\pi_{n}\right)$, and let $\hat{\gamma}$ be a lift of $\gamma$ in $\mathcal{D}\left(\pi_{n}\right)$. The path $\hat{\gamma}$ is not necessarily closed, but a power of $\gamma$ admits a lift $\eta$ which is closed and primitive. Furthermore, $\eta$ consists of a concatenation of lifts of $\gamma$. By Proposition 3.2 the path $\eta$ contains all the letter $0, \ldots, n$ as winner, in particular the letter 0 as winner.

The point is that the letter 0 appears as winner only on the steps of the form $\mathcal{R}_{b}^{k-1}\left(\pi_{n} \circ \sigma^{i}\right) \rightarrow$ $\mathcal{R}_{b}^{k}\left(\pi_{n} \circ \sigma^{i}\right)$. One can assume that $i=0$. By Lemma 3.7, there exists in $\eta$ the step $\mathcal{R}_{b}^{k}\left(\pi_{n}\right) \stackrel{0, *}{\longrightarrow}$ $\mathcal{R}_{b}^{k+1}\left(\pi_{n}\right)$ or the step $\mathcal{R}_{t}^{k}\left(\pi_{n} \circ \sigma^{j}\right) \stackrel{n, *}{\longrightarrow} \mathcal{R}_{t}^{k+1}\left(\pi_{n} \circ \sigma^{j}\right)$, where $j$ corresponds to the index such that $\mathcal{R}_{b} \mathcal{R}_{t}^{k}\left(\pi_{n} \circ \sigma^{j}\right)=\mathcal{R}_{t} \mathcal{R}_{b}^{k}\left(\pi_{n}\right)$.

Since, 0 and $n$ play a symmetric role, we can iterate the argument and therefore, we must reach $k=n$, which corresponds to a central permutation. Hence $\eta$ contains a central permutation. Since $\eta$ is a concatenation of lifts of $\gamma$, this path passes through the reduced permutation $\pi_{n}$. Since $\gamma$ is closed, we can assume that it starts and ends at $\pi_{n}$. Hence, any lift of $\gamma$ has endpoints that are in the preimage of $\pi_{n}$, which are the central permutations. Lemma4.5 is proven.

Proof of Lemma 4.6 Let $\hat{\gamma}$ be a path in $\mathcal{D}\left(\pi_{n}\right)$ connecting two central permutations. For simplicity, assume that $\hat{\gamma}(1)$ is $\pi_{n}$. Again, by symmetry, one can assume that the first arrow is given by the map $\mathcal{R}_{t}$, i.e. $\hat{\gamma}(1) \stackrel{n, 0}{\longrightarrow} \hat{\gamma}(2)=\mathcal{R}_{t}\left(\pi_{n}\right)$. By Lemma 3.7 there exists $i_{2}, \ldots, i_{n-1}$ such that the path $\hat{\gamma}$ contains the steps $\mathcal{R}_{\varepsilon_{2}}^{2}\left(\pi_{n} \circ \sigma^{i_{2}}\right) \rightarrow \mathcal{R}_{\varepsilon_{2}}^{3}\left(\pi_{n} \circ \sigma^{i_{2}}\right), \ldots, \mathcal{R}_{\varepsilon_{n-1}}^{n-1}\left(\pi_{n} \circ \sigma^{i_{n-1}}\right) \rightarrow \mathcal{R}_{\varepsilon_{n-1}}^{n}\left(\pi_{n} \circ \sigma^{i_{n}}\right)$.

The first possibility is that $\hat{\gamma}$ does not change of leaf, i.e. for all $k \in\{2, \ldots, n-1\}, i_{k}=0$. Then the steps previously written form a subpath that can be rewritten as $\hat{\gamma}(1) \stackrel{n, 0}{\longrightarrow} \hat{\gamma}\left(j_{2}\right) \stackrel{n, 1}{\longrightarrow} \hat{\gamma}\left(j_{3}\right) \stackrel{n, 2}{\longrightarrow} \ldots \stackrel{n, n-1}{\longrightarrow}$ $\hat{\gamma}(1)$. Then all the letters $0, \ldots, n-1$ are losers. The path $\hat{\gamma}$ is closed, so $n$ cannot always be winner. Hence it is also looser.

The second possibility is that $\hat{\gamma}$ changes of leaf, i.e. there exists a smallest $k$ such that $i_{k}>0$. Then by Lemma 3.7, a subpath of $\gamma$ is obtained by the following way (compare with Figure 3):

- We start from $\pi_{n}$.

- We apply $k$ times the Rauzy move $\mathcal{R}_{t}$ (these are the moves $\hat{\gamma}(1) \stackrel{n, 0}{\longrightarrow} \hat{\gamma}\left(j_{2}\right) \stackrel{n, 1}{\longrightarrow} \ldots \stackrel{n, k-1}{\longrightarrow}$ $\left.\hat{\gamma}\left(j_{k+1}\right)=\mathcal{R}_{*}^{k}\left(\pi_{n}\right)\right)$. 
- We apply 1 time the Rauzy move $\mathcal{R}_{b}$ (we reach the permutation $\mathcal{R}_{b} \mathcal{R}_{t}^{k}\left(\pi_{n}\right)=\mathcal{R}_{t} \mathcal{R}_{b}^{k}\left(\pi_{n} \circ \sigma^{i_{k}}\right)$ ). This is the move $\gamma\left(j_{k+1}\right) \stackrel{k, n}{\longrightarrow} \hat{\gamma}\left(j_{k+2}\right)$.

- We apply $k^{\prime}=n-k$ times the Rauzy move $\mathcal{R}_{t}$ (until we get the permutation $\mathcal{R}_{b}^{k}\left(\pi_{n} \circ \sigma^{i_{k}}\right)$ ). These are the moves $\hat{\gamma}\left(j_{k+2}\right) \stackrel{1, k}{\longrightarrow} \hat{\gamma}\left(j_{k+3}\right) \stackrel{1, k+1}{\longrightarrow} \ldots \stackrel{1, n-1}{\longrightarrow} \hat{\gamma}\left(j_{n+2}\right)$.

We see that all the letters are losers. This proves Lemma4.6

\section{APPENDIX A. EXAMPLES IN HYPERELLIPTIC COMPONENTS}

In this appendix we show that the uniform lower bound on dilatations of pseudo-Anosov homeomorphisms in Theorem 1.1 is sharp by constructing suitable examples. This will thus give a proof of Theorem 1.2

\section{A.1. Hyperelliptic connected component $\mathcal{H}^{\text {hyp }}(2 g-2)$.}

Proposition A.1. Let $g \geq 2$. There exists a pseudo-Anosov homeomorphism $\phi_{g}$ affine on a translation surface in $\mathcal{H}^{\text {hyp }}(2 g-2)$ whose dilatation is the Perron root of the polynomial $X^{2 g+1}-2 X^{2 g-1}-2 X^{2}+$ 1. This dilatation satisfies

$$
0<\theta\left(\phi_{g}\right)-\sqrt{2}<\frac{1}{2^{g-1}} .
$$

Lemma A.2. The polynomial $P_{g}=X^{2 g+1}-2 X^{2 g-1}-2 X^{2}+1$ admits a unique real root $\alpha$ greater than $\sqrt{2}$.

Proof. $P_{g}(\sqrt{2})=-4+1<0$ and $P_{g}^{\prime}(x)>0$ for $x>\sqrt{2}$.

Lemma A.3. Let $M=\left[m_{i j}\right]$ be the matrix of dimension $2 g$ defined by:

- for all $j \in\{g+1, \ldots, 2 g\}, m_{1, j}=1$, and $m_{1, g}=2$,

- for all $i \in\{2, \ldots, g\}, m_{i, i+g-1}=1$,

- for all $j \in\{g+1, \ldots, 2 g-1\}, m_{i, i-g}=1$,

- $m_{2 g, g}=m_{2 g, 2 g}=1$.

- all the other elements are zero.

$$
M_{g}=\left(\begin{array}{c|ccccc|c} 
& 2 & 1 & \cdots & \cdots & 1 & 1 \\
& 0 & 1 & 0 & \cdots & 0 & 0 \\
0_{g \times g-1} & \vdots & \ddots & \ddots & \ddots & \vdots & \vdots \\
& \vdots & & \ddots & \ddots & 0 & \vdots \\
& 0 & \cdots & \cdots & 0 & 1 & 0 \\
\hline & & & & & & 0 \\
I_{g-1 \times g-1} & & & & & \\
& & & & & & \vdots \\
& & & & & & 0 \\
\hline 0 & 1 & 0 & \cdots & \cdots & 0 & 1
\end{array}\right)
$$

Then $M_{g}$ has $\alpha$ and $1 / \alpha$ as eigenvalues where $\alpha$ is defined by the previous lemma. 
Proof. We must compute the characteristic polynomial $\chi_{g}=\operatorname{det}\left(M_{g}-x I_{2 g \times 2 g}\right)$. Since $P_{g}$ is reciprocal and $\alpha \neq-1$, we just need to show that $(X+1) \chi_{g}=P_{g}$ for all $g \geq 2$. We denote by $L_{i}$ the $i-t h$ line of $M_{g}$. For each $i \in\{1, \ldots, g\}$ we replace $L_{i}$ by $L_{i}+x L_{i+g}$. Then we develop the determinant $g$ times along the first column, to get:

$$
\begin{aligned}
\operatorname{det}\left(M_{g}-x I\right) & =(-1)^{(g+2)(g-1)}\left(2(1-x)+(-1)^{g+2}\left((-1)^{g+1} .1+x(1-x) D_{g-1}\right)\right), \\
& =2(1-x)+(-1)+(-1)^{g} x(1-x) D_{g-1},
\end{aligned}
$$

were $D_{g-1}$ is the $(g-1) \times(g-1)$ determinant:

$$
D_{g-1}=\left|\begin{array}{cccccc}
1-x^{2} & 1 & 1 & \ldots & \ldots & 1 \\
1 & -x^{2} & 0 & \ldots & \ldots & 0 \\
0 & 1 & -x^{2} & 0 & \ldots & 0 \\
\vdots & \ddots & \ddots & \ddots & \ddots & \vdots \\
\vdots & & 0 & 1 & -x^{2} & 0 \\
0 & \ldots & \ldots & 0 & 1 & -x^{2}
\end{array}\right| .
$$

Developing $D_{g}$ on the last column, we obtain $D_{g}=-x^{2} D_{g-1}+(-1)^{g+1}$. Then using this last expression, we see that:

$$
(X+1)\left(\chi_{g+1}-X^{2} \chi_{g}\right)=(X+1)\left(1-X-2 X^{2}+2 X^{3}\right)=\left(2 X^{4}-3 X^{2}+1\right)=P_{g+1}-X^{2} P_{g}
$$

Since $(1+X) \chi_{2}=P_{2}$, we deduce that $(1+X) \chi_{g}=P_{g}$ for all $g \geq 2$, which proves the proposition.

Lemma A.4. Let $\lambda$ (respectively, $\tau$ ) be an eigenvector of $M$ for the eigenvalue $\alpha$ (respectively, $1 / \alpha$ ). Then, up to replacing $\lambda$ or $\tau$ by its opposite, $(\lambda, \tau)$ defines a suspension datum for the permutations $\pi$ and $\pi^{\prime}$, with:

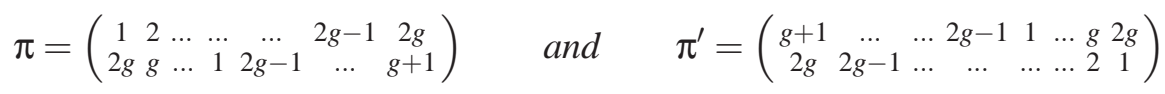

Moreover $(\pi, \lambda, \tau)$ and $\left(\pi^{\prime}, \lambda, \tau\right)$ define the same two surfaces in the moduli space.

Proof. We have $M_{g} \lambda=\alpha \lambda$ and $M_{g} \tau=\frac{1}{\alpha} \tau$. This gives:

$$
\begin{aligned}
& \forall i \in\{1, \ldots, 2 g-2\} \backslash\{g\} \quad \lambda_{i}=\alpha^{2} \lambda_{i+1} \quad \text { and } \quad \tau_{i}=\frac{1}{\alpha^{2}} \tau_{i+1} \\
& \lambda_{2 g-1}=\alpha \lambda_{g} \quad \text { and } \quad \tau_{2 g-1}=\frac{1}{\alpha} \tau_{g} \\
& \lambda_{g}+\lambda_{2 g}=\alpha \lambda_{2 g} \quad \text { and } \quad \tau_{g}+\tau_{2 g}=\frac{1}{\alpha} \tau_{2 g}
\end{aligned}
$$

Since $\alpha>1$, Equation (11) implies that $\lambda_{g}$ is of the same sign as $\lambda_{2 g}$. The other equations imply that all the $\lambda_{i}$ are of the same sign. Hence all the $\lambda_{i}$ are positive if we choose $\lambda_{2 g}>0$.

By a similar argument, for $\tau$, we see that we can choose $\tau_{2 g}<0$ and have $\tau_{i}>0$ for all $i<2 g$. Furthermore, the label " $2 \mathrm{~g}$ " is the last of the first line of $\pi$ (respectively, $\pi^{\prime}$ ) and the first of the second line of $\pi$ (respectively, $\pi^{\prime}$ ). Then in order to prove that $(\lambda, \tau)$ is a suspension data for $\pi$ and $\pi^{\prime}$, it is enough to show that $s=\sum_{i=1}^{2 g} \tau_{i}$ is negative. By Equations (9) and (10), we have

$$
s=\tau_{2 g}+\sum_{k=0}^{2 g-2} \frac{1}{\alpha^{k}} \tau_{g}=\frac{1}{\alpha} \tau_{2 g}+\sum_{k=1}^{2 g-2} \frac{1}{\alpha^{k}} \tau_{g}=\ldots=\frac{1}{\alpha^{2 g-1}} \tau_{2 g}<0 .
$$


Hence we have proven that $(\pi, \lambda, \tau)$ and $\left(\pi^{\prime}, \lambda, \tau\right)$ define translation surfaces by the Veech's construction (see Section 3.2). Now we remark that, if $P_{1}$ is the polygon associated to $(\pi, \lambda, \tau)$, and $P_{2}$ is the polygon associated to $\left(\pi^{\prime}, \lambda, \tau\right)$, then we obtain $P_{2}$ from $P_{1}$ by a $180^{\circ}$ rotation. Since these flat surfaces are hyperelliptic we conclude that the two surfaces defined by $(\pi, \lambda, \tau)$ and $\left(\pi^{\prime}, \lambda, \tau\right)$ are isometric.

Lemma A.5. There is a pseudo-Anosov homeomorphism on the surface defined by the data $(\pi, \lambda, \tau)$ with dilatation $\alpha$.

Proof. We start from $\pi^{\prime}$, and consider the path $\gamma$ in the corresponding Rauzy graph obtained by applying $g$ time the map $\mathcal{R}_{b}$ to $\pi^{\prime}$ and then one time the map $\mathcal{R}_{t}$. We obtain the permutation

$$
\pi^{\prime \prime}=\left(\begin{array}{cccccccc}
g+1 & \ldots & \ldots & 2 g-1 & 1 & \ldots & g & 2 g \\
2 g & 1 & 2 g-1 & \ldots & \ldots & \ldots & \ldots & 2
\end{array}\right) .
$$

The sequence of winners/losers is $(1,2 g),(1, g), \ldots,(1,2),(2 g, 1)$. And therefore, the corresponding transition matrix is:

$$
M_{g}^{\prime}=\left(\begin{array}{ccccc|c|c}
2 & 1 & \cdots & \cdots & 1 & & 1 \\
0 & 1 & 0 & \cdots & 0 & & 0 \\
\vdots & \ddots & \ddots & \ddots & \vdots & 0_{g \times g-1} & \vdots \\
\vdots & & \ddots & \ddots & 0 & & \vdots \\
0 & \cdots & \cdots & 0 & 1 & & 0 \\
\hline & & & & & & 0 \\
& & & & & & \vdots \\
& & 0_{g-1 \times g} & & & I_{g-1 \times g-1} & \vdots \\
& & & & & & 0 \\
\hline 1 & 0 & \cdots & \cdots & 0 & 0 & 1
\end{array}\right)
$$

The permutation $\pi^{\prime \prime}$ is obtained from $\pi$ after renumbering. Multiplying $M^{\prime}$ by the corresponding permutation matrix, one get precisely the matrix $M_{g}$. Let us apply $g+1$ times the Rauzy-Veech induction on $\left(\pi^{\prime}, \lambda, \tau\right)$ to obtain after renumbering $\left(\pi, \frac{1}{\alpha} \lambda, \alpha \tau\right)$. But the two surfaces $X\left(\pi, \frac{1}{\alpha} \lambda, \alpha \tau\right)$ and $X\left(\pi^{\prime}, \frac{1}{\alpha} \lambda, \alpha \tau\right)$ are the same surface by above lemma. Therefore $X\left(\pi^{\prime}, \frac{1}{\alpha} \lambda, \alpha \tau\right)$ and $X\left(\pi^{\prime}, \lambda, \tau\right)$ define also the same surface in the moduli space, hence $X\left(\pi^{\prime}, \lambda, \tau\right)$ admits a pseudo-Anosov homeomorphism with dilatation $\alpha$.

Proof of the proposition. The existence of the pseudo-Anosov homeomorphism $\phi_{g}$ is clear from the previous lemmas. We have $\alpha=\theta\left(\phi_{g}\right)$, and:

$$
\alpha^{2 g+1}-2 \alpha^{2 g-1}-2 \alpha^{2}+1=\alpha^{2}\left(\alpha^{2 g-1}-2 \alpha^{2 g-3}-2\right)+1=0,
$$

thus

$$
\alpha^{2 g-1}-2 \alpha^{2 g-3}-2=\alpha^{2 g-3}\left(\alpha^{2}-2\right)-2<0 .
$$

Since $\alpha>\sqrt{2}$ we obtain $0<\alpha-\sqrt{2}<\frac{2}{(\alpha+\sqrt{2})\left(\alpha^{2 g-3}\right)}<\frac{1}{2^{g-1}}$. 


\section{A.2. Hyperelliptic connected component $\mathcal{H}^{\text {hyp }}(g-1, g-1)$.}

Proposition A.6. Let $g \geq 2$. There exists a pseudo-Anosov homeomorphism on a translation surface in $\mathcal{H}^{\text {hyp }}(g-1, g-1)$ with dilatation the Perron root of the polynomial:

$$
\begin{array}{ll}
X^{2 g+2}-2 X^{2 g}-2 X^{g+1}-2 X^{2}+1, & \text { if } g \text { is even, } \\
X^{2 g+2}-2 X^{2 g}-4 X^{g+2}+4 X^{g}+2 X^{2}-1, & \text { if } g \text { is odd. }
\end{array}
$$

The dilatation satisfies

$$
0<\theta\left(\phi_{g}\right)-\sqrt{2}<\frac{4}{\sqrt{2}^{g}}
$$

Proof. The idea of the proof is very similar to the previous one. We just present here the corresponding paths in the Rauzy diagram.

- If $g$ is even, we start from the permutation $\pi=\left(\begin{array}{ccccccc}1 & \ldots & g+1 & g+2 & \ldots & 2 g & 2 g+1 \\ 2 g+1 & g+1 & \ldots & 1 & 2 g & \ldots & g+2\end{array}\right)$. As previously, we consider the "reverse" permutation $\pi^{\prime}=\left(\begin{array}{cccccccc}g+2 & \ldots & 2 g & 1 & \ldots & g+1 & 2 g+1 \\ 2 g+1 & 2 g & \ldots & g+2 & g+1 & \ldots & 1\end{array}\right)$. Then the path in the Rauzy diagram starting from $\pi^{\prime}$ and defined by $(g+1)$ times $b$, two times $t$. One gets

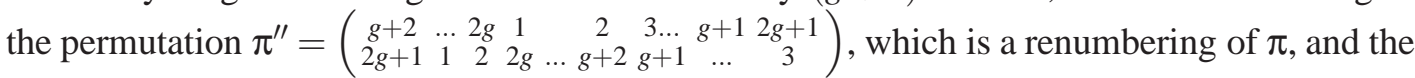
associated matrix is:

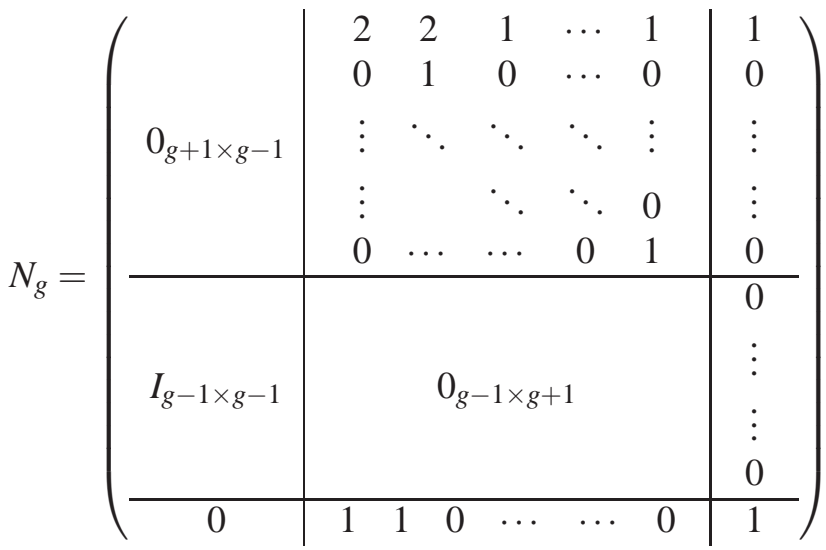

One can check that $(X+1) \chi_{N_{g}}(X)=X^{2 g+2}-2 X^{2 g}-2 X^{g+1}-2 X^{2}+1$, and that, for $\alpha$ the Perron root of $\chi_{N_{g}}(X)$, the eigenvectors of $N_{g}$ corresponding to $\alpha$ and $\frac{1}{\alpha}$ define a suspension data for $\pi$ (and $\pi^{\prime}$ ). Then the corresponding translation surface admits the required pseudoAnosov homeomorphism by construction.

Also, we have

$$
\alpha^{2 g}\left(\alpha^{2}-2\right)<2 \alpha^{g+1}+2 \alpha^{2}
$$

Thus,

$$
\alpha-\sqrt{2}<\frac{2}{\alpha+\sqrt{2}}\left(\frac{1}{\alpha^{g-1}}+\frac{1}{\alpha^{2 g-2}}\right)<\frac{2}{\sqrt{2}^{g}},
$$

since $\alpha>\sqrt{2}$. 
- If $g$ is odd, we consider $\pi$ as previously and we take the path defined by $g$ times $b$, then $t-b-t-t$. We obtain the required pseudo-Anosov with the same construction we presented have above. Also, we have:

$$
\alpha-\sqrt{2}=\frac{1}{\alpha+\sqrt{2}} \frac{4 \alpha^{g}\left(\alpha^{2}-1\right)+1-2 \alpha^{2}}{\alpha^{2 g}}<\frac{4}{\alpha^{g}}<\frac{4}{\sqrt{2}^{g}}
$$

\section{APPENDIX B. EXAMPLES IN NON HYPERELLIPTIC COMPONENTS}

In this appendix we motivate assumptions of Theorem 1.1. We show that if we relax the condition on the hyperelliptic involution, one can construct pseudo-Anosov homeomorphisms in the non hyperelliptic connected components whose dilatations tend to 1 when the genus tends to infinity. This will thus give a proof of Theorem 1.3

We will construct a sequence of pseudo-Anosov homeomorphisms $\varphi_{g}$ on the flat sphere i.e. $\varphi_{g}$ is affine with respect to a quadratic differential $q_{g}$ on the sphere. The construction involves the RauzyVeech induction for quadratic differentials [BL09], using generalized permutations.

Proposition B.1. For each $g \geq 3$, there exists a pseudo-Anosov homeomorphism $\varphi_{g}$ on the flat sphere with $2 g$ poles and two zeroes of order $g-2$, fixing a pole, and having for dilatations $\theta\left(\varphi_{g}\right)$ the Perron root of the polynomial

$$
P=X^{2 g}-X^{2 g-1}-4 X^{g}-X+1 .
$$

Proof of the proposition. We consider the Rauzy diagram corresponding to the stratum of quadratic differentials on the sphere having the given singularity data. It is sufficient to give a closed path in this diagram and to check that the renormalization matrix is irreducible and has $\theta_{g}$ for eigenvalue, where $\theta_{g}$ is the Perron root of $P$.

Let us consider the following generalized permutation (on $2 g+1$ letters)

$$
\left(\begin{array}{cccccccc}
1 & 2 & 2 & 3 & 3 & \ldots & g+1 & g+1 \\
g+2 & g+3 & g+3 & \ldots & 2 g+1 & 2 g+1 & g+2 & 1
\end{array}\right) .
$$

For instance for $g=3$ this gives

$$
\left(\begin{array}{lllllll}
1 & 2 & 2 & 3 & 3 & 4 & 4 \\
5 & 6 & 6 & 7 & 7 & 5 & 1
\end{array}\right)
$$

The Rauzy path we will consider is $t-b-t-b-t-b$. A simple calculation shows that the renormalization matrix $\widehat{V}$ (for the labeled permutation) and permutation matrix $P$ are, respectively

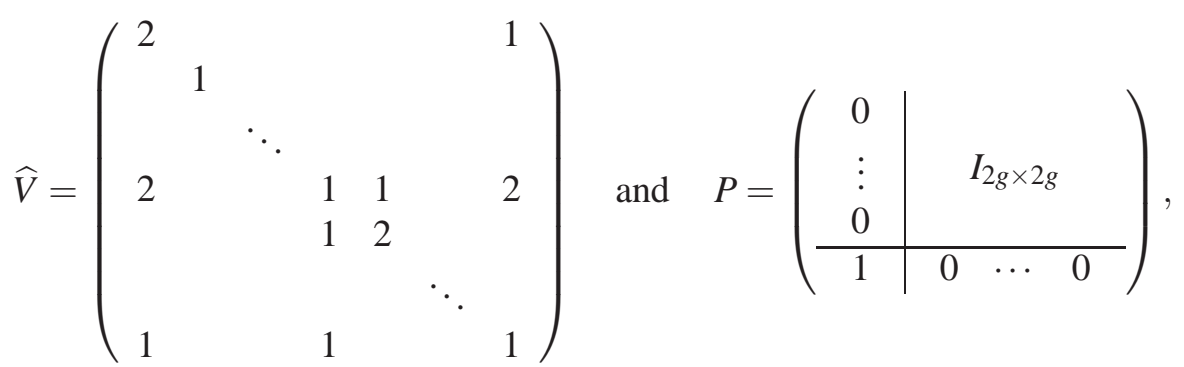


where the "2" in the diagonal of the matrix $\widehat{V}$ appears at the position $g+2$. All the other entries in $\widehat{V}$ are zeroes. Thus the renormalization matrix (for the reduced permutations) is $V=\widehat{V} \cdot P$. One can show that the matrix $V$ is irreducible and its characteristic polynomial is

$$
(X-1)\left(X^{2 g}-X^{2 g-1}-4 X^{g}-X+1\right)=(X-1) P(X) .
$$

Then as in the previous section, we see that some eigenvectors of $V$ define suspension data, and the corresponding flat surface has a pseudo-Anosov homeomorphism whose dilatation is the Perron root of $P$.

Finally we have the following corollary, which justifies the assumptions of Theorem 1.1 on the hyperelliptic involution

Corollary B.2. For $g \geq 3$ odd, there exists an affine pseudo-Anosov homeomorphism $\phi_{g}$ on a translation surface $(X, \omega)$ with the following properties:

(1) $(X, \omega) \in \mathcal{H}^{\text {odd }}(g-1, g-1)$,

(2) $(X, \omega)$ is hyperelliptic,

(3) $\phi_{g}$ fixes a separatrix on $(X, \omega)$ (issued from a Weierstrass point),

(4) the dilatation of $\phi_{g}$ is the Perron root of the polynomial

$$
X^{2 g}-X^{2 g-1}-4 X^{g}-X+1 .
$$

In particular

$$
\lim _{g \rightarrow+\infty} \theta\left(\phi_{g}\right)=1
$$

Remark B.1. Obviously the hyperelliptic involution on $X$ fixes the two zeroes of $\omega$, and $(X, \omega)$ is not in the hyperelliptic connected component of the corresponding stratum.

Proof of the corollary. For $g$ odd, the examples given by Proposition B.1 lift to pseudo-Anosov homeomorphisms $\phi_{g}$ on the orientating cover $(X, \omega) \in \mathcal{H}(g-1, g-1)$. By construction the surface is hyperelliptic and since the poles are ramification points, there is one lift that fixe a regular (Weierstrass) point and the separatrix issued from that point.

To identify the connected component, we use the formula in [Lan04bis]. Since $(X, \omega) \rightarrow\left(\mathbb{P}^{1}, q\right)$ is the orientating cover, the spin structure determined by $(X, \omega)$ is

$$
\left[\frac{\left|n_{+1}-n_{-1}\right|}{4}\right] \quad \bmod 2,
$$

where $n_{+1}$ is the number of singularities of $q$ of degrees $1 \bmod 4$ and $n_{-1}$ is the number of singularities of $q$ of degrees $-1 \bmod 4$.

(1) If $g=1 \bmod 4$ : then $g-2=-1$ modulo 4 , thus $n_{+1}=0$ and $n_{-1}=2 g+2$. Hence the parity of the spin structure on $(X, \omega)$ is (with $g=1+4 k$ )

$$
\left[\frac{\left|n_{+1}-n_{-1}\right|}{4}\right]=\frac{2 g+2}{4}=1+2 k=1 \bmod 2 .
$$


(2) If $g=-1 \bmod 4$ : then $g-2=1$ modulo 4, thus $n_{+1}=2$ and $n_{-1}=2 g$. Hence the parity of the spin structure on $(X, \omega)$ is (with $g=-1+4 k$ )

$$
\left[\frac{\left|n_{+1}-n_{-1}\right|}{4}\right]=\frac{2 g-2}{4}=-1+2 k=1 \bmod 2 .
$$

\section{REFERENCES}

[AY81] P. ARnOuX and J. C. YocCOZ - "Construction de difféomorphismes pseudo-Anosov (French)", C. R. Acad. Sci. Paris Sér. I Math. 292 (1981), pp. 75-78.

[AGY06] A. Avila, S. GouËzEl and J.-C. YocCOZ - "Exponential mixing for the Teichmüller flow", Publ. Math. Inst. Hautes Études Sci. No. 104 (2006), pp. 143-211.

[AV07] A. AVILA and M. ViAnA - "Simplicity of Lyapunov spectra: proof of the Zorich-Kontsevich conjecture", Acta Math. 198 No. 1 (2007), pp. 1-56.

[BL09] C. BOISSY and E. LANNEAU - "Dynamics and geometry of the Rauzy-Veech induction for quadratic differentials", Ergodic Theory Dynam. Systems 29 (2009), no. 3, pp. 767-816.

[Boi09] C. BoIssy - "Classification of Rauzy classes in the moduli space of quadratic differentials. ", preprint arXiv:0904.3826 (2009).

[Boi10] , "Labeled Rauzy classes and framed translation surfaces ", in preparation.

[Buf06] A. BufETOV - "Decay of correlations for the Rauzy-Veech-Zorich induction map on the space of interval exchange transformations and the central limit theorem for the Teichmüller flow on the moduli space of abelian differentials ", J. Amer. Math. Soc. 19 (2006), no. 3, pp. 579-623.

V. DELECROIX - "Cardinality of Rauzy classes", in preparation (2010).

[CH08] J. CHO and J. HAM - "The minimal dilatation of a genus two surface", Experiment. Math. 17 (2008), pp. 257267.

[Del10] V. DeleCroiX - "Cardinality of Rauzy classes”, preprint (2010).

[Far06] B. FARB - "Some problems on mapping class groups and moduli space", Problems on Mapping Class Groups and Related Topic ed. by B. Farb, Proc. Symp. Pure and Applied Math. 74, (2006) pp. 11-55.

[FLM08] B. FARB, C. Leininger and D. MARGALit - "The lower central series and pseudo-Anosov dilatations", American Journal of Mathematics 130 (2008), pp. 799-827.

[FK92] H.M. FARKA and I. KRA - "Riemann surfaces, 2nd ed.", Grad. Text Math. 71 Springer-Verlag, Berlin, (1992).

[FLP79] A. FAthi, F. LAudenbach and V. PoÉnARU - "Travaux de Thurston sur les surfaces", Astérisque 66-67 (1979).

[Hir09] E. HiRONAKA - "Small dilatation pseudo-Anosov mapping classes coming from the simplest hyperbolic braid", preprint arXiv:0909.4517(2009).

[HM79] J. HUBBARD and H. MASUR - "Quadratic differentials and foliations ", Acta Math. 142 (1979), no. 3-4, pp. 221274.

[Iva88] N.V. IVANOV - "Coefficients of expansion of pseudo-Anosov homeomorphisms", Zap. Nauchn. Sem. Leningrad. Otdel. Mat. Inst. Steklov (LOMI) 167 (1988), translation in J. Soviet Math. 52 (1990), pp. 2819-2822.

[Ker85] S. KERCKHOFF - "Simplicial systems for interval exchange maps and measured foliations ", Ergodic Theory Dynam. Systems 5 (1985), no. 2, pp. 257-271.

[KZ03] M. KONTSEVICH and A. ZORICH - "Connected components of the moduli spaces of Abelian differentials with prescribed singularities", Invent. Math. 153 (2003), no. 3, pp. 631-678.

[KT10] E. KIN and M. TAKASAWA - "Pseudo-Anosovs on closed surfaces having small entropy and the Whitehead sister link exterior", preprint [ps] arXiv:1003.0545](2010).

[FL87] J. FEhrEnBACH and J. LOS - "Une minoration de l'entropie topologique des difféomorphismes du disque (French) ", J. London Math. Soc. 60 (1999), no. 3, 912-924.

[Lan04] E. LANNEAU - "Hyperelliptic components of the moduli spaces of quadratic differentials with prescribed singularities", Comment. Math. Helv. 79 (2004), pp. 471-501. 
[Lan04bis] __ Parity of the spin structure defined by a quadratic differential, Geom. Topol. 8 (2004) 511-538 (electronic).

[Lan08] _ "Connected components of the strata of the moduli spaces of quadratic differentials with prescribed singularities", Ann. Sci. École Norm. Sup. (4) 41 (2008), pp. 1-56.

[Lan10] _ "An Infinite sequence of fixed point free pseudo-Anosov homeomorphisms on a genus two surface", Contemporary Mathematics (2010).

[LT10] E. LANNEAU and J-L. ThiffEAUlT - "On the minimum dilatation of pseudo-Anosov homeomorphisms on surfaces of small genus", Annales Fourier 60 (2010).

[Lei04] C. J. LEININGER - "On groups generated by two positive multi-twists: Teichmüller curves and Lehmer number", Geom. Topo. 8 (2004), pp. 1301-1359.

[Los09] J. Los - "Infinite sequences of fixed point free pseudo-Anosov homeomorphisms.", Erg. Th. Dyn. Sys, to appear (2009).

[MMY05] S. MARmi, P. Mouss a and J.-C. YocCOZ - "The cohomological equation for Roth type interval exchange transformations", Journal of the Amer. Math. Soc. 18 (2005), pp. 823-872.

[MS93] H. MASUR and J. SMILliE - "Quadratic differentials with prescribed singularities and pseudo-Anosov homeomorphisms”, Comment. Math. Helv. 68 (1993), pp. 289-307.

[MT02] H. MASUR, S. TABAChniKov-“Rational billiards and flat structures", Handbook of dynamical systems, 1A, North-Holland, Amsterdam (2002), pp. 1015-1089.

[McM00] C. T. MCMULLEN - "Polynomial invariants for fibered 3-manifolds and Teichmüller geodesics for foliation ", Ann. Sci. École Norm. Sup. 33 (4) (2000), pp. 519-560.

[Pen91] R. Penner - "Bounds on least dilatations", Proc. Amer. Math. Soc. 113 (1991), pp. 443-450.

[HP91] R. PEnNeR and J. HARER - "Combinatorics of Train Tracks", Annals of Mathematics Studies 125, Princeton University Press (1991).

[Ste09] W. A. Stein ET AL - "Sage Mathematics Software (Version 4.2.1)", The Sage Development Team, 2009, http://www.sagemath.org

[Rau79] G. RAUZY - "Échanges d'intervalles et transformations induites", Acta Arith. 34 (1979), pp. 315-328.

[Thu88] W. ThURSTON - "On the geometry and dynamics of homeomorphisms of surfaces", Bull. A.M.S. 19 (1988), pp. 417-431.

[Tsa09] C. TSAI - "The asymptotic behavior of least pseudo-Anosov dilatations", Geometry \& Topology 13 (2009), pp. 2253-2278.

[Vee82] W. VEECH - "Gauss measures for transformations on the space of interval exchange maps", Ann. of Math. (2) 115 (1982), no. 1, pp. 201-242.

[Vee89] _ "Teichmüller curves in modular space, Eisenstein series, and an application to triangular billiards", Inv. Math. 97, (1989) 553-583.

Laboratoire d’Analyse, Topologie et Probabilité, Université Paul CéZanne, Faculté de Saint Jérôme, AVENue ESCADrille Normandie-NiEMEn, CASE COUR A, 13397 MARSEILlE CEDEX 20, FRANCE

E-mail address: corentin.boissy@latp.univ-mrs.fr

Centre de Physique Théorique (CPT), UMR CNRS 6207

UNIVERSITÉ DU SUD TOULON-VAR AND

FÉdération de ReCherches des Unités de Mathématiques de Marseille

Luminy, CASE 907, F-13288 Marseille CEDEX 9, FranCE

E-mail address: lanneaudcpt . univ-mrs.fr 


\title{
PSEUDO-ANOSOV HOMEOMORPHISMS ON TRANSLATION SURFACES IN HYPERELLIPTIC COMPONENTS HAVE LARGE ENTROPY
}

\author{
CORENTIN BOISSY, ERWAN LANNEAU
}

\begin{abstract}
We prove that the dilatation of any pseudo-ANOSOV homeomorphism on a translation surface that belong to a hyperelliptic component is bounded from below uniformly by $\sqrt{2}$. This is in contrast to PENNER's asymptotic. PENNER proved that the logarithm of the least dilatation of any pseudo-ANOSOV homeomorphism tends to zero at rate $1 / g$ (as $g$ goes to infinity).

We also show that our uniform lower bound $\sqrt{2}$ is sharp. The proof uses the RAUZY-VEECH induction.
\end{abstract}

\section{INTRODUCTION}

Pseudo-Anosov homeomorphisms play an important role in TeICHMÜLLER theory. Calculating problems for their dilatations have a long history in differential geometry. The unit cotangent bundle of the moduli space of compact genus $g$ RIEMANN surfaces $\mathcal{M}_{g}$ can be viewed as the moduli space of quadratic differentials $Q_{g} \rightarrow \mathcal{M}_{g}$. This space is naturally stratified by strata of quadratic differentials with singularities of prescribed multiplicities. The TEICHMÜLLER geodesic flow $g_{t}$ acts naturally on these strata, and closed loops of length $\log (\theta)>0$ for this flow correspond to conjugacy classes of Pseudo-ANOSOV homeomorphisms with dilatation $\theta>1$. An important problem concerns the asymptotic behavior of the smallest dilatations.

The strata are not necessarily connected (see KonTSEVICH \& ZORICH [KZ03] and LANNEAU [Lan08]). In this paper we use a discretization of the TEICHMÜLLER geodesic flow, i.e. the RAUZY-VEECH induction, in order to tackle the minimisation problem for hyperelliptic components $C^{\text {hyp }}$. This is the first instance of asymptotic for components of the moduli spaces. We shall prove

Theorem. Let $g \geq 1$. Let $\delta\left(C^{\text {hyp }}\right)$ be the least dilatation of pseudo-ANOSOV homeomorphisms affine on translation surfaces that belong to $C^{\text {hyp }} \subset Q_{g}$. Then

$$
\sqrt{2}<\delta\left(C^{h y p}\right)<\sqrt{2}+\frac{4}{\sqrt{2}^{g}} .
$$

We will give a more precise statement in the following.

Date: May 22, 2010.

2000 Mathematics Subject Classification. Primary: 37E05. Secondary: 37D40.

Key words and phrases. Pseudo-ANOSOV homeomorphisms, Interval exchange transformations, RAUZYVEECH induction, Moduli spaces. 
Mapping class group. The mapping class $\operatorname{group} \operatorname{Mod}(S)$ of a closed orientable surface $S$ of genus $g \geq 1$ is defined to be the group of homotopy classes of orientation preserving homeomorphisms of $S$. An irreducible mapping class is an isotopy class of homeomorphisms so that no powers preserve a nontrivial subsurface of $S$. By the THURSTON-NIELSEN classification [Thu88], irreducible mapping classes are either periodic (analogous to roots of unity) or are of a type called pseudo-ANOSOV [FLP79]. To each pseudo-ANOSOV mapping class $[\phi] \in \operatorname{Mod}(S)$ one can attach a dilatation factor $\theta(\phi)>1$. The logarithm of $\theta(\phi)$ can be viewed as the minimal topological entropy of any element in the homotopy class of $\phi$ (uniquely realized by some element, $\phi)$.

THURSTON proved that this number is an algebraic integer and even a PERRON number. $\theta(\phi)>1$ is also the exponential growth rate of lengths of curves under iteration of $\phi$ (in any metric on $S$ ). These numbers appear naturally as the length spectrum of the moduli space of genus $g$ RIEMANN surfaces.

It is an open question to characterize the set of dilatations of pseudo-ANOSOV homeomorphisms. THURSTON has conjectured that pseudo-ANOSOV dilatations (ignoring genus) are precisely the algebraic units that are PERRON and also larger than the GALOIS conjugates of their inverses.

Minimisation problem. Since the set of dilatations (for fixed genus) is discrete [AY81, Iva88] (as a subset of $\mathbb{R}$ ) the least dilatation $\delta_{g}$ is well defined. We know very little about the values of the constants $\delta_{g}$. The precise value of $\delta_{2}$ has been recently calculated (see CHO \& HAM [CH08] and LANNEAU \& THIFFEAULT [LT10]) but the values of $\delta_{g}$ for $g \geq 3$ are still unknown.

Upper bounds are not hard to derive from examples and there are a lot of results in that direction (see e.g. Penner, McMullen, Hironaka, Kin, Minakawa). So far the best general upper bound is the one given by HIRONAKA \& KIN [HK06]: $\log \left(\delta_{g}\right) \leq \frac{\log (2+\sqrt{3})}{g}$. See also [Hir09, KT10] for recent results in that direction. But again very little is known about lower bounds. PENNER [Pen91] proved that $\log \left(\delta_{g}\right) \geq \frac{\log (2)}{12 g-12}$, using general properties of the PERRON-Frobenius matrices. There is also a result of TSAI [Tsa09] for pseudo-ANOSOV on punctured surfaces.

In general lower bounds are much subtle to obtain than upper bounds. In contrast to our understanding of the asymptotic of $\log \left(\delta_{g}\right)$, we still do not know the answer to the following question, posed by MCMuLLEN [McM00, Section 10]:

Does $\lim _{g \rightarrow+\infty} g \log \left(\boldsymbol{\delta}_{g}\right)$ exist? What is its value?

Subgroups of the modular group and strata. In his book [Far06], FARB proposed two natural refinements of the minimisation problem.

The first one is related to subgroups of the modular group. More precisely, let us fix a subgroup $H \subseteq \operatorname{Mod}(S)$ and let us consider the least dilatation $\delta(H)$ of pseudo-Anosov classes $[\phi] \in H$. 
In order to state the second problem, let us recall the definition of strata here (see Section 2 for precise statements). A pseudo-ANOSOV homeomorphism on a compact surface defines a pair of transverse measured foliations. For simplicity, we will assume that the foliations are orientable, but the techniques we will develop holds for non-orientable measured foliations (see Section 2.3). These data are equivalent to the given of a pair $(X, \omega)$ (called a translation surface for a reason that will be clear in the next) where $X$ is a Riemann surface and $\omega$ is a holomorphic 1 -form with zeroes of multiplicities $\left(k_{1}, \ldots, k_{n}\right)$. We will also say singularities of multiplicities $\left(k_{1}, \ldots, k_{n}\right)$ of the translation surface. The GAUSS-BONNET formula reads $\sum_{i=1}^{n} k_{i}=2 g-2$. A stratum consists of the set of translation surfaces with prescribed singularities. MASUR \& SMILLIE [MS93] proved that, for every singularity data, there exits some pseudo-ANOSOV homeomorphism that realize it. Hence it is natural to consider the following problem (minimisation problem in a stratum)

$$
\delta\left(k_{1}, \ldots, k_{n}\right):=\inf \left\{\log (\phi): \begin{array}{l}
\text { pseudo-ANOSOV } \phi \text { whose corresponding } \\
\text { translation surface has singularity data }\left(k_{1}, \ldots, k_{n}\right)
\end{array}\right\} .
$$

Similarly, for a pair of nonoriented measured foliation, one gets a pair $(X, q)$, where $X$ is a Riemann surface and $q$ is a holomorphic quadratic differential with zeroes of order $\left(k_{1}, \ldots, k_{n}\right)$. Obviously one has

$$
\delta_{g}=\min \left\{\delta\left(k_{1}, \ldots, k_{n}\right)\right\}
$$

where the min is taken over all possible singularity data (with no assumptions on the orientability of the foliations).

It turns out that strata (translation surfaces with prescribed singularity data $\left(k_{1}, \ldots, k_{n}\right)$ ) are not connected in general [KZ03, Lan08]. We can thus consider the natural following refinement of FARB's question : minimizing problem for connected components.

The components are distinguished by two invariants the parity of the spin structure and the hyperelliptic components. The latter are defined as follows.

Recall that a RIEMANN surface $X$ of genus $g \geq 2$ is hyperelliptic if there exists an holomorphic involution $\tau$ with $2 g+2$ fixed points.

Convention. In all of this paper, we will use the following convention: a flat surface $(X, \omega)$ is hyperelliptic if the underlying RIEMANN surface is hyperelliptic and $\tau^{*} \omega=-\omega$ (or equivalently is an affine homeomorphism, see Section 2.1). The fixed points of $\tau$ will be usually called WEIERSTRASS points.

Hyperelliptic connected components $C^{\text {hyp }}$ are defined in the following way

$$
C^{\text {hyp }}:=\left\{(X, \omega): \begin{array}{l}
X \text { is hyperelliptic; } \omega \text { is a holomorphic one form with one single } \\
\text { and permutes the zeroes, if there are two }
\end{array}\right\} .
$$

LEININGER [Lei04] and then FARB, LEININGER \& MARGALIT [FLM08] tackle the minimisation problem for the subgroups of $\operatorname{Mod}(S)$ given by the THURSTON's construction and for the 
TORELLI group, respectively. They provide evidence for the principle that algebraic complexity implies dynamical complexity.

In this paper we will investigate the second problem and prove a similar theorem. This answers a question of FARB [Far06, Problem 7.5]. We shall prove

Theorem 1.1. Let $g \geq 1$. Let $\phi$ be a pseudo-ANOSOV homeomorphism affine on a genus $g$ translation surface $(X, \omega) \in C^{\text {hyp }}$. Then

$$
\theta(\phi)>\sqrt{2}
$$

Recall that PENNER [Pen91] proved that, as the genus increases, there are pseudo-ANOSOV homeomorphisms with dilatations arbitrarily close to 1 .

This uniform lower bound is sharp. Indeed one can construct (see Appendix A) a sequence $\left(\phi_{g}\right)_{g \geq 1}$ on $(X, \omega) \in C^{\text {hyp }}$ with dilatations converging to $\sqrt{2}$. More precisely, we will show

Theorem 1.2. Let $g \geq 1$. Let $\delta\left(C^{\text {hyp }}\right)$ be the least dilatation of pseudo-Anosov homeomorphisms affine on a genus $g$ translation surface $(X, \omega) \in C^{\text {hyp }}$. Then

$$
\sqrt{2}<\delta\left(C^{\text {hyp }}\right)<\sqrt{2}+\frac{4}{\sqrt{2}^{g}} .
$$

Non hyperelliptic components. The reader may wonder why we impose the restrictions on the action of the hyperelliptic involution on the zeroes in the definition of $C^{\text {hyp }}$. It turns out that if we don't impose this restriction, then the translation surface is hyperelliptic, but not in the hyperelliptic connected component, and therefore, the property of being hyperelliptic is not preserved by any small deformation inside the ambiant stratum. We will show that the asymptotic behavior may be very different if we consider different connected components.

As for example one can construct (see Appendix B) a sequence $\left(\varphi_{g}\right)_{g \geq 3}$ (with $g$ odd) of pseudo-ANOSOV homeomorphisms on a hyperelliptic translation surface $\left(X_{g}, \omega_{g}\right)$ of genus $g$ having two zeroes of degree $g-1, g-1$, and such that the dilatation of $\varphi_{g}$ is the PERRON root of the polynomial

$$
X^{2 g}-X^{2 g-1}-4 X^{g}-X+1
$$

In particular

$$
\lim _{k \rightarrow+\infty} \theta\left(\varphi_{2 k+1}\right)=1
$$

Of course in that case the hyperelliptic involution fixes the two zeroes.

In the above case, the (non-hyperelliptic) components are distinguished by a parity of the spin structure (see [KZ03] and Section 2.2). Let $C^{\text {odd }}$ be the (non-hyperelliptic) odd component. Then we shall prove 
Theorem 1.3. Let $g \geq 1$, be an odd integer. Let $\delta\left(C^{o d d}\right)$ be the least dilatation of pseudoANOSOV homeomorphisms affine on a genus g translation surface $(X, \omega) \in C^{\text {odd }}$. Then

$$
1<\delta\left(C^{\text {odd }}\right)<1+\frac{1}{g}
$$

Quadratic differentials. A quadratic differential $(X, q)$ is strictly quadratic if it is not the square of any holomorphic 1-form. $q$ determines foliations with singularities ( $p$-prongs). There exists a canonical branched double cover $Y \rightarrow X$ on which $q$ becomes a square. The branch points correspond to the simple poles (1-prongs) and zeros of odd order of $q$. We will prove Theorem 1.1 for Abelian differentials or equivalently translation surfaces.

In Section 2.3 we derive from our theorem some results for strict quadratic differentials, namely we will prove Theorem 2.1 page 9

As mentioned, one has a more general result, with no assumptions whether the foliations are orientable or not.

Theorem 1.4. Let $g \geq 1$. Let $\phi$ be a pseudo-ANOSOV homeomorphism on a genus $g$ hyperelliptic surface. Let us assume that the foliations have some 1-prongs and only one or two p-prongs with $p \geq 2$. Assume also that the hyperelliptic involution is affine (for the flat metric). If the involution permutes the singularities (if there are two) then

$$
\theta(\phi)>\sqrt{2} .
$$

RAUZY-VEECH induction. If $C$ is a connected component of some strata then there is a finite ramified covering $\widehat{C} \rightarrow \mathcal{C}$ consisting of marking a zero and a separatrix of surfaces in $\mathcal{C}$. The RAUZY-VEECH induction provides a discrete representation (symbolic coding) of the TEICHMÜLLER flow on $\widehat{C}$ [Vee82] (see Section 3 for precise definitions). Periodic orbits in $C$ are taken to conjugacy classes of pseudo-ANOSOV on $(X, \omega) \in \mathcal{C}$.

As shown by VEECH, to each periodic orbit $\gamma \subset \widehat{C}$ there corresponds a closed loop in some graph (called a RAUZY diagram) $\mathcal{D}_{r}(C)$ and a renormalization matrix $V(\gamma) \in S L(h, \mathbb{Z})(h=2 g+n-1)$. This matrix corresponds to the action of the corresponding pseudo-ANOSOV homeomorphism in relative homology of the underlying surface with respect to the singularities of the Abelian differential. Hence the spectral radius of $V(\gamma)$ is the dilatation of the pseudo-AnOSOV on $(X, \omega) \in \mathcal{C}$.

A crucial point in this paper is a carefully analysis of the geometry of these RAUZY diagrams. This approach was already used by AVILA \& VIANA [AV07] for the dynamical properties of the TEICHMÜLLER geodesic flow.

Outline of a proof of our main result. The RAUZY-VEECH induction allows one to relate pseudo-ANOSOV homeomorphisms and closed loops in RAUZY diagrams. We conclude by sketching its use in the proof of Theorem 1.1 . 
1. Let $\phi$ be a pseudo-ANOSOV homeomorphism affine with respect to a translation surface $(X, \omega) \in C^{\text {hyp }}$. We prove that $\phi$ commutes with the hyperelliptic involution (Proposition 2.2). Thus $\phi$ induces a pseudo-ANOSOV homeomorphism on the sphere.

2. Using the BROUWER fixed point theorem, we show that $\phi^{2}$ fixes a separatrix of the horizontal measured foliation on $X$ (Proposition 4.1).

3. Hence $\phi^{2}$ is also affine with respect to a surface $(X, \omega) \in \widehat{C^{\text {hyp }}}$ (with a marked separatrix). Thus $\phi^{2}$ is obtained by taking a (irreducible) closed loop in the RAUZY diagram $\mathcal{D}_{r}^{\text {hyp }}$ corresponding to $\widehat{C^{h y p}}$.

4. The transition matrices of the diagram $\mathcal{D}_{r}^{\text {hyp }}$ are hard to describe. We use MARMIMOUSSA-YOCCOZ's representation which furnished a covering $\mathcal{D}^{\text {hyp }} \rightarrow \mathcal{D}_{r}^{\text {hyp }}$. We then obtain a simple criterion for a path $\gamma$ (not necessarily closed) in $\mathcal{D}^{\text {hyp }}$ to have a transition matrix $V(\gamma)$ with spectral radius greater than 2 (Proposition 4.2).

5. Analyzing carefully the combinatorics of these RAUZY diagrams $\mathcal{D}^{\text {hyp }}$, we show that $\theta\left(\phi^{2}\right) \geq 2$ (see Section 3.7.2). Since $\theta\left(\phi^{2}\right)=\theta(\phi)^{2}$ one gets the desired result.

6. In Appendix $\mathrm{A}$ we prove that our uniform bound is sharp by exhibiting a sequence of pseudo-ANOSOV homeomorphisms. In Appendix B we show that the action of the hyperelliptic involution on the zeroes is important.

Further results. FEHRENBACH \& LOS [FL87] proved the following inequality for pseudoANOSOV homeomorphisms $\phi$ having a periodic orbit of length $n \geq 3: \log (\phi) \geq \frac{1}{n} \log (1+\sqrt{2})$. However this does not easily imply a uniform lower bound. Indeed for each hyperelliptic connected component, there exists a pseudo-ANOSOV homeomorphism such that the minimal length (greater than two) of a periodic orbit is the number of WEIERSTRASS points i.e. $2 g+2$ points (see the appendix). In particular, Theorem 1.1 does not follow from these estimates.

See also [Los09, Lan10] for further results on pseudo-ANOSOV that do not come from the VEECH's construction.

Acknowledgements. We thank Vincent DELECROIX for remarks and comments on this paper and for his very useful sage's library "IET" [Ste09].

\section{BACKGROUND}

We review basic notions and results concerning Abelian differentials, translation surfaces, pseudo-ANOSOV homeomorphisms and moduli spaces. For general references see say [MT02, Vee82, Rau79, MS93, MMY05].

2.1. Flat surfaces and pseudo-A Nosov homeomorphisms. A flat surface $S$ is a (real, compact, connected) genus $g$ surface equipped with a flat atlas i.e. a triple $(S, \mathcal{U}, \Sigma)$ such that $\Sigma$ is a finite subset of $S$ (whose elements are called singularities) and $\mathcal{U}=\left\{\left(U_{i}, z_{i}\right)\right\}$ is an atlas of $S \backslash \Sigma$ with transition maps $z \mapsto \pm z+$ constant. We will require that for each $s \in \Sigma$, there is a 
neighborhood of $s$ isometric to a Euclidean cone. Therefore we get a quadratic differential defined locally in the coordinates $z_{i}$ by the formula $q=d z_{i}^{2}$. This form extends to the points of $\Sigma$ to zeroes, simple poles or marked points (we will usually call the zeroes and poles singular points or simply singularities).

If there exists a sub-atlas such that all transition functions are translations then the quadratic differential $q$ is the global square of an Abelian differential $\omega \in H^{1}(X, \mathbb{C})$. We will then say that $(X, \omega)$ is a translation surface.

A homeomorphism $f: X \rightarrow X$ is an affine homeomorphism if $f$ restricts to a diffeomeomorphism of $X \backslash \Sigma$ of constant derivative. It is equivalent to say that $f$ restricts to an isomorphism of $X \backslash \Sigma$ which preserves the induced affine structure given by $q$.

There is a standard classification of elements of $\mathrm{SL}_{2}(\mathbb{R})$ into three types: elliptic, parabolic and hyperbolic. This induces a classification of affine diffeomorphisms. An affine diffeomorphism is parabolic, or elliptic, or pseudo-Anosov, respectively, if $|\operatorname{trace}(D f)|=2$, $|\operatorname{trace}(D f)|<2$, or $|\operatorname{trace}(D f)|>2$, respectively. If $\phi$ is pseudo-ANOSOV, in the coordinates of the stable and unstable measured foliations determined by $\phi$, one has $D \phi=\left(\begin{array}{cc}\theta^{-1} & 0 \\ 0 & \theta\end{array}\right)$ where $|\theta|>1$. The number $|\theta|$ is called the dilatation of $\phi$. From now all flat surfaces considered will be translation surfaces, except in Section 2.3 and Appendix B.

Remark 2.1. Constructing parabolic elements is easy (see [Vee89]) but constructing hyperbolic elements is more subtle. A first construction is to consider the product of parabolic elements (see e.g. the paper of FATHI [Fat87]). In Section 3 we recall a very general construction due to VEECH.

For $g \geq 1$, we define the moduli space of Abelian differentials $\mathcal{H}_{g}$ as the moduli space of pairs $(X, \omega)$ where $X$ is a genus $g$ (compact, connected) RIEMANN surface and $\omega \in \Omega(X)$ a non-zero holomorphic 1 -form defined on $X$. The term moduli space means that we identify the points $(X, \omega)$ and $\left(X^{\prime}, \omega^{\prime}\right)$ if there exists an analytic isomorphism $f: X \rightarrow X^{\prime}$ such that $f^{*} \omega^{\prime}=\omega$. The group $\mathrm{SL}_{2}(\mathbb{R})$ naturally acts on the moduli space of flat surfaces by post composition on the charts.

One can also see a translation surface obtained as a polygon (or a union of polygons) whose sides come by pairs, and for each pairs, the corresponding segments are parallel and of the same lengths. These parallel sides are glued together by translation and we assume that this identification preserves the natural orientation of the polygons. In this context, two translation surfaces are identified in the moduli space of Abelian differentials if and only if the corresponding polygons can be obtained from each other by "cutting" and "gluing" and preserving the identifications (i.e. the two surfaces represent the same point in the moduli space). Also, the $S L_{2}(\mathbb{R})$ action in this representation is just the natural linear action on the polygons.

VEECH showed that an affine homeomorphism with a derivative map which is not the identity is not isotopic to the identity. Hence a homeomorphism $f$ is an affine homeomorphism on the 
flat surface $(X, \omega)$, with derivative map $D f=A$, if and only if the matrix $A$ stabilizes the surface $(X, \omega)$. That is $(X, \omega)$ can be obtained from $A \cdot(X, \omega)$ by "cutting" and "gluing".

2.2. Connected components of the strata. The moduli space of Abelian differentials is stratified by the combinatorics of the zeroes; We will denote by $\mathcal{H}\left(k_{1}, \ldots, k_{r}\right)$ the stratum of $\mathcal{H}_{g}$ consisting of (classes of) pairs $(X, \omega)$ such that $\omega$ possesses exactly $r$ zeroes on $X$ with multiplicities $\left(k_{1}, \ldots, k_{r}\right)$.

It is a well known part of the TEICHMÜLLER theory that these spaces are (HAUSDORFF) complex analytic, and in fact algebraic, spaces.

These strata are non-connected in general but each stratum has at most three connected components (see [KZ03] for a complete classification). In particular for $g \geq 4$ the stratum with a single zero, $\mathcal{H}(2 g-2)$, has three connected components. The stratum $\mathcal{H}(g-1, g-1)$ has two or three connected components depending whether $g$ is even or odd, respectively.

2.2.1. Hyperelliptic component. This component contains precisely pairs $(X, \omega)$ where $X$ is a hyperelliptic surface and $\omega$ a one-form whose zeroes (if there are two) are interchanged by the hyperelliptic involution. An equivalent formulation is to require that there exists a ramified double cover $\pi: X \rightarrow \mathbb{P}^{1}$ over the sphere and a quadratic differential $q$ on $\mathbb{P}^{1}$ having only one zero and simples poles such that $\omega^{2}=\pi^{*} q$. We will denote these components by $\mathcal{H}^{\text {hyp }}(2 g-2)$ and $\mathcal{H}^{\text {hyp }}(g-1, g-1)$.

2.2.2. Other components. The other (non-hyperelliptic) components are distinguished by a parity of the spin structure. There are two ways to compute the parity of the spin structure of a translation surface $X$. The first way is to use the ARF formula on a symplectic basis (see [KZ03]). The second possibility applies if $X$ comes from a quadratic differential, i.e. if $X$ possesses an involution such that the quotient produces a half-translation surface [Lan04bis]. We will apply this in Appendix B.

2.3. Application of Theorem 1.1 to quadratic differentials. In this section, we extend Theorem 1.1 to some other strata in the moduli space of quadratic differentials. This part is independent from the rest of the paper and can be skipped for a first reading. However, Proposition 2.2 will be needed later.

As for Abelian differentials, strata of the moduli space of quadratic differentials are not connected in general (see [Lan08] for a complete classification). We can deduce from Theorem 1.1 results on hyperelliptic components in the quadratic case.

We denote by $Q\left(k_{1}, \ldots, k_{n}\right)$ strata of the moduli space of half translation surfaces where the vector $\left(k_{1}, \ldots, k_{n}\right)$ agrees with the GAUSS-BONNET formula $\sum_{i=1}^{n} k_{i}=4 g-4$. For $g \geq 2$, let us consider the two strata $Q(-1,-1,2 g-3,2 g-3)$ and $Q(-1,-1,4 g-2)$. Their hyperelliptic component can be defined as follows (see [Lan04]):

$$
C_{1}^{\text {hyp }}:=\left\{(X, q): \begin{array}{l}
X \text { is hyperelliptic, }(X, q) \in Q(-1,-1,2 g-3,2 g-3), \text { and } \\
\text { the involution permutes the two zeroes and the two poles }
\end{array}\right\} .
$$




$$
C_{2}^{\text {hyp }}:=\left\{(X, q): \begin{array}{l}
X \text { is hyperelliptic, }(X, q) \in Q(-1,-1,4 g-2), \text { and } \\
\text { the involution permutes the two poles }
\end{array}\right\} .
$$

Theorem 2.1. Let $g \geq 2$. Let $\mathcal{C}$ be one of the two hyperelliptic components defined above. Let $\phi$ be a pseudo-ANOSOV homeomorphism affine on a half translation surface $(X, q) \in \mathcal{C}$. Then the dilatation $\theta(\phi)$ satisfies $\theta(\phi)>\sqrt{2}$.

We will use the following result.

Proposition 2.2. Let $f$ be an affine homeomorphism on hyperelliptic flat surface of genus greater than or equal to two. The map $f$ commutes with the hyperelliptic involution.

Proof of Proposition 2.2 Let $\tau$ be the hyperelliptic involution on $X$. The commutator $[f, \tau]=$ $f \circ \tau \circ f^{-1} \circ \tau$ is an affine homeomorphism and its derivative map is the identity matrix in $\mathrm{SL}_{2}(\mathbb{R})$. So $[f, \tau]$ is a translation.

Since the genus is greater than or equal to two, and the surface is hyperelliptic, then the set of WEIERSTRASS points is nonempty. We show that $f$ preserves set-wise the set of WEIERSTRASS points (i.e. the set $W=\operatorname{Fix}(\tau)$ ). The map $f^{-1} \tau f$ is a conformal automorphism of the complex surface, it is easy to see that it is a hyperelliptic involution. Hence it preserves point-wise the WEIERSTRASS points [FK92]. Let $p \in W$. Then, $f^{-1} \tau f(p)=p$ so $\tau f(p)=f(p)$. Hence $f(p)$ is a fixed point of $\tau$ thus $f(p) \in W$. Since $f$ preserves set-wise $W$ the translation $[f, \tau]$ fixes point-wise $W$. It is easy to see that it preserves also the separatrices issued from the regular WEIERSTRASS points, that necessarily exists. Thus the commutator $[f, \tau]$ is the identity. The proposition is proven.

Proof of Theorem 2.1] Let $(X, q) \in Q(-1,-1,2 g-3,2 g-3)$ be a half translation surface in the hyperelliptic component. Let $\phi$ be a pseudo-ANOSOV homeomorphism on $X$ and $\tau$ the hyperelliptic involution.

Passing to the quotient we get a meromorphic quadratic differential $q^{\prime}$ on the projective line $\mathbb{P}^{1}$ with $2 g+1$ simple poles and a single zero of degree $2 g-3$. Taking the standard orientating cover $\pi: Y \rightarrow \mathbb{P}^{1}$ over $\mathbb{P}^{1}$ having ramification points precisely over odd degree singularities (namely the poles and the zero) we obtain a translation surface $(Y, \omega)$ where $\omega^{2}=\pi^{*} q^{\prime}$. By construction (see Subsection 2.2.1) $(Y, \omega)$ belongs to the hyperelliptic component $\mathcal{H}^{h y p}(2 g-2)$. Now by Proposition 2.2 $\phi$ commutes with $\tau$ on $Y$. Thus $\phi$ induces a pseudo-ANOSOV homeomorphism $\varphi$ on $\mathbb{P}^{1}$ with the same dilatation. Since $\varphi$ preserves the set of ramification points of $\pi$, $\varphi$ lifts to a new pseudo-ANOSOV homeomorphism, say $\hat{\varphi}$, on $Y$.

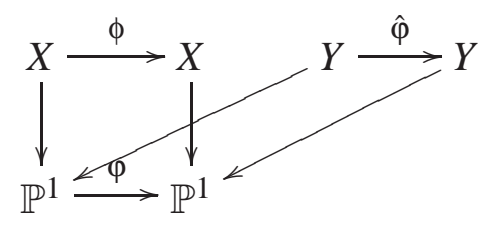

Now $\theta(\phi)=\theta(\varphi)=\theta(\hat{\varphi})$. By Theorem 1.1 we get that $\theta(\phi)>\sqrt{2}$. 
The second case is similar and left to the reader.

We end with the following (see the introduction):

Theorem 1.4, Let $g \geq 1$. Let $\phi$ be a pseudo-ANOSOV homeomorphism on a genus $g$ hyperelliptic surface. Let us assume that the foliations have some 1-prongs and only one or two p-prongs with $p \geq 2$. Assume also that the hyperelliptic involution is affine (for the flat metric). If the involution permutes the singularities (if there are two) then

$$
\theta(\phi)>\sqrt{2}
$$

Proof of Theorem 1.4 Let $\pi:(X, \omega) \rightarrow\left(\mathbb{P}^{1}, q\right)$ be the covering. By construction $\phi$ induces a pseudo-ANOSOV homeomorphism on the sphere and lift to a pseudo-ANOSOV homeomorphism on a translation surface in some hyperelliptic component, with the same dilatation. Then Theorem 1.1 applies.

\section{RAUZY-VEECH INDUCTION AND PSEUDO-ANOSOV HOMEOMORPHISMS}

In this section we recall the basic construction of pseudo-ANOSOV homeomorphisms using the RAUZY-VEECH induction (for details see [Vee82], §8, and [Rau79, MMY05]). We first review the link between interval exchange maps and translation surfaces.

3.1. Interval exchange transformations. Let $I \subset \mathbb{R}$ be an open interval and let us choose a finite partition of $I$ into $d \geq 2$ open subintervals $\left\{I_{j}, j=1, \ldots, d\right\}$. An interval exchange transformation is a one-to-one map $T$ from $I$ to itself that permutes, by translation, the subintervals $I_{j}$. It is easy to see that $T$ is precisely determined by the following data: a permutation that encodes how the intervals are exchanged, and a vector with positive entries that encodes the lengths of the intervals.

We will use the description given by MARMI, Moussa \& YocCOZ [MMY05]. This will simplify the description of the induction which will be very useful for the proof of our result.

We will attribute a name to each interval $I_{j}$. In this case, we will speak of labeled interval exchange maps. One gets a pair of one-to-one maps $\left(\pi_{t}, \pi_{b}\right)$ (t for "top" and b for "bottom") from a finite alphabet $\mathcal{A}$ to $\{1, \ldots, d\}$ in the following way. In the partition of $I$ into intervals, we denote the $k^{\text {th }}$ interval, when counted from the left to the right, by $I_{\pi_{t}^{-1}(k)}$. Once the intervals are exchanged, the interval number $k$ is $I_{\pi_{b}^{-1}(k)}$. Then with this convention, the permutation encoding

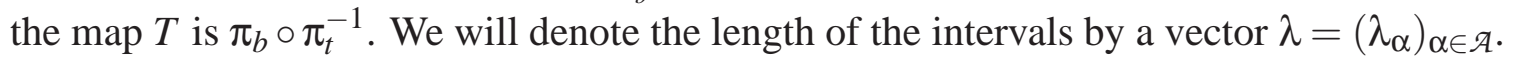

Definition 3.1. We will call the pair $\left(\pi_{t}, \pi_{b}\right)$ a labeled permutation, and $\pi_{b} \circ \pi_{t}^{-1}$ a permutation (or reduced permutation). If it is clear from the context, then we will just use the term permutation. We will also usually write a reduced permutation as a labeled one with $\mathcal{A}=\{1, \ldots, d\}$ and $\pi_{t}=I d$. 
One usually represents labeled permutations $\pi=\left(\pi_{t}, \pi_{b}\right)$ by a table:

$$
\pi=\left(\begin{array}{cccc}
\pi_{t}^{-1}(1) & \pi_{t}^{-1}(2) & \ldots & \pi_{t}^{-1}(d) \\
\pi_{b}^{-1}(1) & \pi_{b}^{-1}(2) & \ldots & \pi_{b}^{-1}(d)
\end{array}\right) .
$$

3.2. Suspension data. The next construction provides a link between interval exchange transformations and translation surfaces. A suspension datum for $T=(\pi, \lambda)$ is a collection of vectors $\left\{\tau_{\alpha}\right\}_{\alpha \in \mathcal{A}}$ such that

- $\forall 1 \leq k \leq d-1, \sum_{\pi_{t}(\alpha) \leq k} \tau_{\alpha}>0$

- $\forall 1 \leq k \leq d-1, \sum_{\pi_{b}(\alpha) \leq k} \tau_{\alpha}<0$.

We will often use the notation $\zeta=(\lambda, \tau)$. To each suspension datum $\tau$, we can associate a translation surface $(X, \omega)=X(\pi, \zeta)$ in the following way.

Consider the broken line $L_{t}$ on $\mathbb{C}=\mathbb{R}^{2}$ defined by concatenation of the vectors $\zeta_{\pi_{t}^{-1}(j)}$ (in this order) for $j=$ $1, \ldots, d$ with starting point at the origin. Similarly, we consider the broken line $L_{b}$ defined by concatenation of the vectors $\zeta_{\pi_{b}^{-1}(j)}$ (in this order) for $j=1, \ldots, d$ with starting point at the origin. If the lines $L_{t}$ and $L_{b}$ have no intersec-

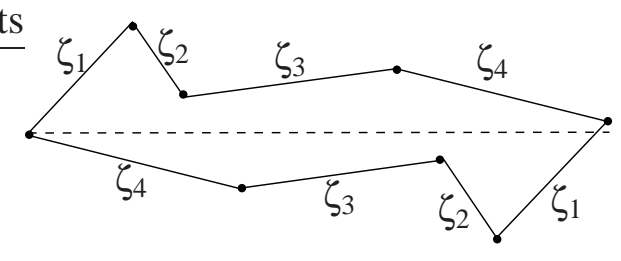
tions other than the endpoints, we can construct a translation surface $X$ by identifying each side $\zeta_{j}$ on $L_{t}$ with the side $\zeta_{j}$ on $L_{b}$ by a translation. The resulting surface is a translation surface endowed with the form $d z^{2}$. Note that the lines $L_{t}$ and $L_{b}$ might have some other intersection points. But in this case, one can still define a translation surface by using the zippered rectangle construction, due to VEECH ([Vee82]).

Let $I \subset X$ be the horizontal interval defined by $I=\left(0, \sum_{\alpha} \lambda_{\alpha}\right) \times\{0\}$. Then the interval exchange transformation $T$ is precisely the one defined by the first return map of the vertical flow on $X$ to $I$.

3.3. RAUZY-VEeCH induction. The RAUZY-VEeCH induction $\mathcal{R}(T)$ of $T$ is defined as the first return map of $T$ to a certain subinterval $J$ of $I$ (see [Rau79, MMY05] for details).

We recall very briefly the construction. Following [AGY06] we define the type of $T$ by $t$ if $\lambda_{\pi_{t}^{-1}(d)}>\lambda_{\pi_{b}^{-1}(d)}$ and $b$ if $\lambda_{\pi_{t}^{-1}(d)}<\lambda_{\pi_{b}^{-1}(d)}$. When $T$ is of type $t$ (respectively, $b$ ) we will say that the label $\pi_{t}^{-1}(d)$ (respectively, $\pi_{b}^{-1}(d)$ ) is the winner and that $\pi_{b}^{-1}(d)$ (respectively, $\pi_{t}^{-1}(d)$ ) is the looser. We define a subinterval $J$ of $I$ by

$$
J= \begin{cases}I \backslash T\left(I_{\pi_{b}^{-1}(d)}\right) & \text { if } T \text { is of type t; } \\ I \backslash I_{\pi_{t}^{-1}(d)} & \text { if } T \text { is of type b. }\end{cases}
$$

The image of $T$ by the RAUZY-VEECH induction $\mathcal{R}$ is defined as the first return map of $T$ to the subinterval $J$. This is again an interval exchange transformation, defined on $d$ letters (see e.g. [Rau79]). The data of $\mathcal{R}(T)$ are very easy to express in term of those of $T$.

There are two cases to distinguish depending whether $T$ is of type $t$ or $b$; the labeled permutations of $\mathcal{R}(T)$ only depends on $\pi$ and on the type of $T$. If $\varepsilon \in\{t, b\}$ is the type of $T$, this defines 
two maps $\mathcal{R}_{t}$ and $\mathscr{R}_{b}$ by $R(T)=\left(\mathcal{R}_{\varepsilon}(\pi), \lambda^{\prime}\right)$. We will often make use of the following notation: if $\varepsilon \in\{t, b\}$ we denote by $1-\varepsilon$ the other element of $\{t, b\}$.

(1) $T$ has type $t$. Let $k \in\{1, \ldots, d-1\}$ such that $\pi_{b}^{-1}(k)=\pi_{t}^{-1}(d)$. Then $\mathcal{R}_{t}\left(\pi_{t}, \pi_{b}\right)=\left(\pi_{t}^{\prime}, \pi_{b}^{\prime}\right)$ where $\pi_{t}=\pi_{t}^{\prime}$ and

$$
\pi_{b}^{\prime-1}(j)= \begin{cases}\pi_{b}^{-1}(j) & \text { if } j \leq k \\ \pi_{b}^{-1}(d) & \text { if } j=k+1 \\ \pi_{b}^{-1}(j-1) & \text { otherwise }\end{cases}
$$

(2) $T$ has type $b$. Let $k \in\{1, \ldots, d-1\}$ such that $\pi_{t}^{-1}(k)=\pi_{b}^{-1}(d)$. Then $\mathcal{R}_{b}\left(\pi_{t}, \pi_{b}\right)=$ $\left(\pi_{t}^{\prime}, \pi_{b}^{\prime}\right)$ where $\pi_{b}=\pi_{b}^{\prime}$ and

$$
\pi_{t}^{\prime-1}(j)= \begin{cases}\pi_{t}^{-1}(j) & \text { if } j \leq k \\ \pi_{t}^{-1}(d) & \text { if } j=k+1 \\ \pi_{t}^{-1}(j-1) & \text { otherwise. }\end{cases}
$$

(3) Let us denote by $E_{\alpha \beta}$ the $d \times d$ matrix of which the $\alpha, \beta$-th element is equal to 1 , all others to 0 . If $T$ is of type $t$ then let $(\alpha, \beta)=\left(\pi_{t}^{-1}(d), \pi_{b}^{-1}(d)\right)$ otherwise let $(\alpha, \beta)=$ $\left(\pi_{b}^{-1}(d), \pi_{t}^{-1}(d)\right)$. Then if $V_{\alpha \beta}$ is the transvection matrix $I+E_{\alpha \beta}$ then $V_{\alpha \beta} \lambda^{\prime}=\lambda$.

Remark 3.1. In the VEECH's original construction, the matrices used to obtain $\lambda^{\prime}$ in terms of $\lambda$ were more complicated: of the form $P+E_{\alpha \beta}$ where $P$ is a permutation matrix. Indeed, after the RAUZY induction "bottom", we usually have $\pi_{t}^{\prime} \neq I d$, and we must "renumber" it.

This construction is due to RAUZY. This induction is called the RAUZY-VEECH induction since VEECH observed that one can actually define the induction on the suspension data in the following way. If $\tau$ is a suspension data over $(\pi, \lambda)$ then we define $R(\pi, \lambda, \tau)$ by

$$
\mathcal{R}(\pi, \lambda, \tau)=\left(\mathcal{R}_{\varepsilon}(\pi), V^{-1} \lambda, V^{-1} \tau\right)
$$

where $\varepsilon$ is the type of $T=(\pi, \lambda)$ and $V$ is the corresponding transition matrix. In other terms $V_{\alpha \beta} \zeta^{\prime}=\zeta$ where $\zeta=(\lambda, \tau)$.

Remark 3.2. By construction the two translation surfaces $X(\pi, \zeta)$ and $X\left(\pi^{\prime}, \zeta^{\prime}\right)$ are naturally isometric (as translation surfaces).

Now if we iterate the RAUZY induction, we get a sequence $\left(\alpha_{k}, \beta_{k}\right)$ of winners/loosers. If $\mathcal{R}^{(n)}(\pi, \lambda)=\left(\pi^{(n)}, \lambda^{(n)}\right)$ then the transition matrix that rely $\lambda^{(n)}$ to $\lambda$ is the product of the transition matrices:

$$
\left(\prod_{k=1}^{n} V_{\alpha_{k} \beta_{k}}\right) \lambda^{(n)}=\lambda
$$

For a labeled permutation $\pi$, we call the labeled RAUZY diagram, denoted by $\mathcal{D}(\pi)$, the graph whose vertices are all labeled permutations that we can obtained from $\pi$ by the combinatorial RAUZY moves. From each vertices, there are two edges labeled $t$ and $b$ (the type) corresponding 
to the two combinatorial RAUZY moves. We will denote by $\pi \stackrel{\alpha, \beta}{\longrightarrow} \pi^{\prime}$ for the edge corresponding to $\mathcal{R}_{\varepsilon}(\pi)=\pi^{\prime}$ where $\varepsilon \in\{t, b\}$ and $\alpha / \beta$ is the winner/looser. To each path $\gamma$ is this diagram, there is thus a sequence of winners/loosers. We will denote by $V(\gamma)$ the product of the transition matrices in Equation (1). The next lemma is clear from the definition.

Lemma 3.1. Let $\gamma_{n}=\pi_{1} \ldots \pi_{n}$ be a path in the labeled RAUZY diagram, and let $V_{n}$ be the matrix associated to the path $\gamma_{n}$. Let $\alpha, \beta$ be the winner/looser associated to the edge $\pi_{n-1} \stackrel{\alpha, \beta}{\longrightarrow} \pi_{n}$. Then $V_{n}$ is obtained from $V_{n-1}$ by adding the column $\alpha$ to the column $\beta$.

Definition 3.2. A closed path in the labeled RAUZY diagram is said to be primitive if the associated matrix $V$ is primitive, i.e. if there exists a power of $V$ such that all the entries are positive. We will also say that a path contains the letter $\alpha$ as winner (respectively, looser) if it contains the edge $\cdot \stackrel{\alpha, \beta}{\longrightarrow} \cdot($ respectively, $\cdot \stackrel{\beta, \alpha}{\longrightarrow} \cdot$ ), for some $\beta$.

We have the following proposition (see [MMY05], proposition in section 1.2.3),

Proposition 3.2 (MARMi, Moussa \& YocCOZ). A closed path $\gamma$ in a labeled RAUZY diagram is primitive if and only if $\gamma$ contains all the letters as winner at least once.

3.4. Reduced RAUZY diagrams. We have previously defined RAUZY induction and RAUZY diagrams for labeled interval exchange transformations. One can also define the same for reduced interval exchange transformations, as it was first, for which the corresponding labeled permutation is just a permutation of $\{1, \ldots, d\}$ (see [Vee82]). These are obtained after identifying $\left(\pi_{t}, \pi_{b}\right)$ with $\left(\pi_{t}^{\prime}, \pi_{b}^{\prime}\right)$ if $\pi_{b} \circ \pi_{t}^{-1}=\pi_{b}^{\prime} \circ \pi_{t}^{\prime-1}$. In the next we will use the notation $\mathcal{D}_{r}(\pi)$ to denote the reduced RAUZY diagram associated to the permutation $\pi$.

Note that the labeled RAUZY diagram is naturally a covering of the reduced RAUZY diagram, and they are usually not isomorphic.

Given a closed path $\gamma$ in the reduced RAUZY diagram, as previously, one can associate a matrix $V$ as follow: we take $\left(\pi_{t}, \pi_{b}\right)$ the labeled permutation corresponding to the endpoint of $\gamma$ so that $\pi_{t}=I d$. Then we consider $\hat{\gamma}$ a lift of $\gamma$ in the labeled RAUZY diagram. The path $\hat{\gamma}$ is not necessarily closed and it ends at a permutation $\left(\pi_{t}^{\prime}, \pi_{b}^{\prime}\right)$. We can associate to it a matrix $\widehat{V}$ as before. Let $P$ be the permutation matrix defined by permuting the columns of the $d \times d$ identity matrix according to the permutation $\pi_{t}^{\prime}$, i.e. the $P=\left[p_{i j}\right]$, with $p_{i j}=1$ if $j=\pi_{t}^{\prime}(i)$ and 0 otherwise. The transition matrix associated to the path is then:

$$
V=\widehat{V} \cdot P \text {. }
$$

As before, a closed path in the reduced RAUZY diagram is called primitive if $V$ is primitive. A standard reference for the next two sections is [Vee82].

3.5. Construction of pseudo-Anosov homeomorphisms. There is a natural $\mathrm{SL}_{2}(\mathbb{R})$-action on the strata. In particular, the one parameter subgroup $g_{t}=\left(\begin{array}{cc}e^{t} & 0 \\ 0 & e^{-t}\end{array}\right)$ is called the TEICHMÜLLER geodesic flow. It can be shown that conjugacy classes of pseudo-ANOSOV homeomorphisms are 
one-to-one with closed geodesics of the TEICHMÜLLER geodesic flow on strata. There is a very nice construction of pseudo-ANOSOV homeomorphisms using the RAUZY-VEECH induction, and we recall now this construction.

Let $\pi$ be an irreducible permutation and let $\gamma$ be a closed loop in the reduced RAUZY diagram associated to $\pi$. One can associate to $\gamma$ a matrix $V(\gamma)$ (see section above). Let us assume that $V$ is primitive and let $\theta>1$ be its PERRON-FROBENIUS eigenvalue. We choose a positive eigenvector $\lambda$ for $\theta$. It can be shown that $V$ is symplectic [Vee82], thus let us choose an eigenvector $\tau$ for the eigenvalue $\theta^{-1}$ with $\tau_{\pi_{0}^{-1}(d)}>0$. It turns out that $\tau$ defines a suspension data over $T=(\pi, \lambda)$. Indeed, the set of suspension data is an open cone, that is preserved by $V^{-1}$. Since the matrix $V^{-1}$ has a dominant eigenvalue $\theta$ (for the eigenvector $\tau$ ), the vector $\tau$ must belong to this cone. If $\zeta=(\lambda, \tau)$, one has

$$
\begin{array}{r}
\mathcal{R}(\pi, \zeta)=\left(\pi, V^{-1} \zeta\right)=\left(\pi, V^{-1} \lambda, V^{-1} \tau\right)=\left(\pi, \theta^{-1} \lambda, \theta \tau\right)= \\
=g_{t}(\pi, \lambda, \tau), \quad \text { where } \quad t=\log (\theta)>0 .
\end{array}
$$

Hence the two surfaces $X(\pi, \zeta)$ and $g_{t} X(\pi, \zeta)$ differ by some element of the mapping class group (see Remark 3.2). In other words there exists a pseudo-ANOSOV homeomorphism $\phi$ affine with respect to the translation surface $X(\pi, \zeta)$ and such that $D \phi=g_{t}$. The action of $\phi$ on the relative homology of $(X, \omega)$ is $V(\gamma)$ thus the dilatation of $\phi$ is $\theta$. Note that by construction $\phi$ fixes the zero on the left of the interval $I$ and also a horizontal separatrix adjacent to this zero (namely, the oriented half line corresponding to the interval $I$ ).

It turns out that this construction is very general as we will see in the coming section.

3.6. Discrete representation of the geodesic flow. Let us fix an irreducible permutation $\pi$ defined over $d$ letters. If $\mathcal{C}$ is the connected component of some stratum, let $\widehat{C}$ be the ramified cover over $\mathcal{C}$ obtained by considering the set of triplets $(X, \omega, l)$ where $(X, \omega) \in \mathcal{C}$ and $l$ is a horizontal separatrix adjacent to a zero of $\omega$.

Clearly the set of $(\lambda, \tau)$ such that $\tau$ is a suspension data over $\pi$ is a connected space and the map $(\lambda, \tau)=\zeta \mapsto X(\pi, \zeta)$ is continuous. Thus all surfaces obtained by this construction belong to the same connected component of some strata, say $\mathcal{C}(\pi) \subset \mathcal{H}(\sigma)$. Moreover $\sigma$ can be computed easily in terms of $\pi$. We define

$$
\mathcal{T}(\widehat{C})=\{(\pi, \zeta) ; \widehat{C}(\pi)=\widehat{C}, \text { and } \zeta \text { is a suspension datum for } \pi\}
$$

The RAUZY-VEECH induction is (almost everywhere) well defined and one-to-one on $\mathcal{A}(\widehat{\mathcal{C}})$. Hence let $\mathcal{H}(\widehat{C})$ be the quotient of $\mathcal{T}(\widehat{\mathcal{C}})$ by the induction. 
The VEECH zippered rectangle's construction provides (almost everywhere) a one-to-one map $Z: \mathcal{H}(\widehat{C}) \rightarrow \widehat{C}($ see [Boi09] for details).

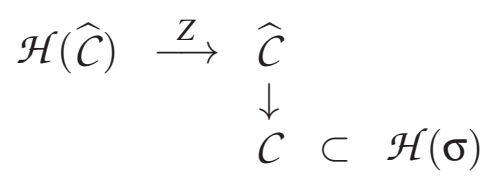

One can define the TEICHMÜLLER geodesic flow on $\mathcal{H}(\widehat{\mathcal{C}})$ by $g_{t}(\pi, \zeta)=\left(\pi, g_{t} \zeta\right)$. The TEICHMÜLLER flow on $\mathcal{C}$ lifts to a flow $g_{t}$ on $\widehat{\mathcal{C}}$. It is easy to check that $g_{t}$ is equivariant with $Z$ i.e. $g_{t} Z=Z g_{t}$.

By construction, periodic orbits of $g_{t}$ on $\mathcal{C}$ corresponding to pseudo-ANOSOV homeomorphisms that fix the separatrix $I$ lift to periodic orbits on $\widehat{C}$ for $g_{t}$. Thus they produce periodic orbits for $g_{t}$ on suspensions $\mathcal{H}(\widehat{\mathcal{C}})$.

In fact VEECH proved more: all pseudo-ANOSOV homeomorphisms fixing a separatrix arise in this way. The subset of $\mathcal{T}(\widehat{C})$ defined by

$$
\left\{(\pi, \zeta) \in \mathcal{T}(\widehat{\mathcal{C}}) ; 1 \leq|\operatorname{Re}(\zeta)| \leq 1+\min \left(\operatorname{Re}\left(\zeta_{\pi_{0}^{-1}(d)}\right), \operatorname{Re}\left(\zeta_{\pi_{1}^{-1}(d)}\right)\right)\right\}
$$

is a fundamental domain of $\mathcal{T}(\widehat{\mathcal{C}})$ for the quotient map $\mathcal{T}(\widehat{\mathcal{C}}) \rightarrow \mathcal{H}(\widehat{\mathcal{C}})$, and the POINCARÉ map of the TEICHMÜLLER flow on the section

$$
\mathcal{S}=\{(\pi, \zeta) ; \pi \text { irreducible, }|\operatorname{Re}(\zeta)|=1\} / \sim
$$

is precisely the renormalized RAUZY-VEECH induction on suspensions:

$$
\widehat{\mathcal{R}}(\pi, \lambda):=\left(\mathcal{R}_{\varepsilon}(\pi), V^{-1} \lambda /\left|V^{-1} \lambda\right|\right) .
$$

We can summarize the above discussion by the following theorem.

Theorem (VEECH). Let $\gamma$ be a closed loop, based at $\pi$, in a reduced RAUZY diagram $\mathcal{D}_{r}(\pi)$ and let $V=V(\gamma)$ be the product of the associated transition matrices. Let us assume that $V$ is primitive. Let $\lambda$ be a positive eigenvector for the PERRON-FROBENIUS eigenvalue $\theta$ of $V$ and let $\tau$ be an eigenvector for the eigenvalue $\theta^{-1}$ of $V$ with $\tau_{\pi_{0}^{-1}(1)}>0$. Then

(1) $\zeta=(\lambda, \tau)$ is a suspension datum for $T=(\pi, \lambda)$;

(2) The matrix $A=\left(\begin{array}{cc}\theta^{-1} & 0 \\ 0 & \theta\end{array}\right)$ is the derivative map of an affine pseudo-ANOSOV homeomorphism $\phi$ on $X(\pi, \zeta)$; The action on relative homology of $\phi$ is the matrix $V$.

(3) The dilatation of $\phi$ is $\theta$;

(4) All pseudo-ANOSOV homeomorphisms that fix a separatrix are obtained in this way.

We will use this theorem in order to prove our main result. 


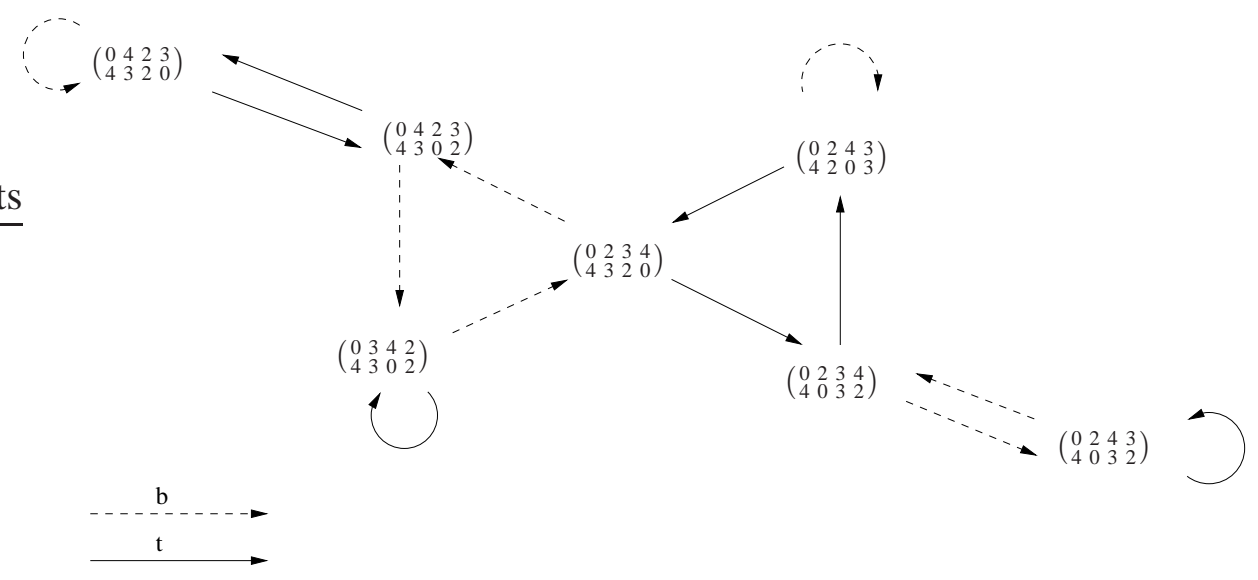

FIGURE 1. The diagram $\mathcal{D}^{h y p}=\mathcal{D}\left(\tau_{n}\right) \approx \mathcal{D}_{r}\left(\tau_{n}\right)$ for $n=4$.

\subsection{Examples of labeled and reduced RAUZY diagrams.}

Convention. Let $\pi$ be a permutation. We will denote by $\mathcal{D}(\pi)$ the labeled RAUZY diagram of $\pi$ and by $\mathcal{D}_{r}(\pi)$ the reduced one.

3.7.1. Hyperelliptic connected components. Let $n \geq 2$. A representative permutation for the connected component $\mathcal{H}^{\text {hyp }}(2 g-2)$ (respectively, $\mathcal{H}^{\text {hyp }}(g-1, g-1)$ ) is

$$
\tau_{n}=\left(\begin{array}{cccccc}
0 & 2 & 3 & \ldots & n-1 & n \\
n & n-1 & \ldots & 3 & 2 & 0
\end{array}\right),
$$

where $n=2 g$ (respectively, $n=2 g+1$ ). We use this permutation in order to simplify the notations latter. It turns out that labeled and reduced RAUZY diagrams are isomorphic. The precise description of the diagrams was given by RAUZY [Rau79]. Let us recall the result here.

If $\pi=\left(\pi_{t}, \pi_{b}\right)$ is a labeled permutation, for $\varepsilon \in\{t, b\}$ we define $G^{\varepsilon}(\pi)$ to be the subdiagram of the RAUZY diagram of $\pi$ whose vertices are obtained from $\pi$ by a simple path, and whose first step is the map $\mathcal{R}_{\varepsilon}$. Recall that for $\varepsilon \in\{t, b\}$, we denote by $1-\varepsilon$ the other element of $\{t, b\}$.

Proposition (RAUZY). Let $\tau_{n}$ be the labeled permutation defined in Equation (3). Then

(1) The vertices of the RAUZY diagram of $\tau_{n}$ is the disjoint union of the vertices of $G^{0}\left(\tau_{n}\right)$, $G^{1}\left(\tau_{n}\right)$, and $\left\{\tau_{n}\right\}$.

(2) Let $\tau_{n, k}=\mathcal{R}_{\varepsilon}^{k}\left(\tau_{n}\right)$, for some $\varepsilon \in\{t, b\}$ and $k \in\{1, \ldots, n-1\}$. Then $G^{1-\varepsilon}\left(\tau_{n, k}\right)$ is naturally isomorphic to $G^{1-\varepsilon}\left(\tau_{n-k}\right)$.

(3) The cardinality of the RAUZY diagram is $2^{n-1}-1$.

Using this result, one can show that the labeled and reduced diagrams are isomorphic. We will denote by $\mathcal{D}^{h y p}=\mathcal{D}\left(\tau_{n}\right) \approx \mathcal{D}_{r}\left(\tau_{n}\right)$ this RAUZY diagram. A consequence of the previous proposition is the following: 
Corollary 3.3. Let $\gamma$ be a closed (oriented) path in $\mathcal{D}^{\text {hyp }}$. Assume that it contains the step $\pi \stackrel{\alpha, \beta}{\longrightarrow} \pi^{\prime}$ with $\pi \neq \pi^{\prime}$, then it must contain the step $\pi^{\prime} \stackrel{\alpha, \beta^{\prime}}{\longrightarrow} \pi^{\prime \prime}$.

Proof. A consequence of the RAUZY description of $\mathcal{D}^{\text {hyp }}$ is that $\mathcal{D}^{\text {hyp }} \backslash\left\{\pi^{\prime}\right\}$ is not connected any more. Assume that, in the step following $\pi \stackrel{\alpha, \beta}{\longrightarrow} \pi^{\prime}$, the symbol $\alpha$ is looser, then the new permutation and $\pi$ belong to two different connected components of $\mathcal{D}^{\text {hyp }} \backslash \pi^{\prime}$. Looking at the sequence of permutations that appear, one must come back to the connected component of $\pi$, and hence, the step $\pi^{\prime} \stackrel{\alpha, \beta^{\prime}}{\longrightarrow} \pi^{\prime \prime}$ eventually appears.

3.7.2. Hyperelliptic connected components with a marked point. Here we present a second family of RAUZY diagrams that will be useful for our proof. There description is a little bit more technical, but will be needed later. We start with a very informal description of the labeled RAUZY diagram. The precise description can be skipped in a first reading.

The labeled RAUZY diagram is a covering of the reduced one. The cardinalities of these labeled RAUZY diagrams have been calculated (see DELECROIX [Del10]). The degree of the covering is also known [Boi10].

A fundamental domain of this covering can be roughly seen as a copy of the hyperelliptic RAUZY diagram described previously, with some added permutations (see Figure 2). This fundamental domain (a leaf) is composed at first glance by two principal loops that intersect in a central permutation, and on the over vertices of these loops starts a secondary loop. The whole diagram is obtained by taking several copies of this "leaf". The different leaves are joined together by the transition permutations: each secondary loops contains a unique such transition permutation, and a "k-th secondary loop" of a leaf is attached to a "k-th secondary loop" of another leaf (see Figure 3, and compare it with Figure 2).

We now give the precise description of this diagram. A representative permutation for the connected component $\mathcal{H}^{\text {hyp }}(0,2 g-2)$ (respectively, $\mathcal{H}^{\text {hyp }}(0, g-1, g-1)$ ) is

$$
\pi_{n}=\left(\begin{array}{ccccccc}
0 & 2 & 3 & \ldots & n-1 & 1 & n \\
n & n-1 & \ldots & 3 & 2 & 1 & 0
\end{array}\right),
$$

where $n=2 g$ (respectively, $n=2 g+1$ ). Contrary to the previous case, the diagrams labeled and reduced are not isomorphic anymore. As we will see the cardinality of the reduced diagram is $2^{n-1}-1+n$ and the cardinality of the labeled diagram is $\left(2^{n-1}-1+n\right)(n-1)$.

Our next goal is to describe the reduced RAUZY diagrams $\mathcal{D}\left(\pi_{n}\right)$ and $\mathcal{D}_{r}\left(\pi_{n}\right)$ associated to the above permutation $\pi_{n}$. The key point is to observe that if we forbid the letter 1 to be winner or looser in the construction of the diagram $\mathcal{D}\left(\pi_{n}\right)$ starting from $\pi$, then one gets more or less the diagram $\mathcal{D}^{\text {hyp }}$ (compare with AVILA \& ViAnA AV07]). So we have to add the letter 1 to the permutations in $\mathcal{D}^{\text {hyp }}$. One can do that as follows.

(1) To each permutation $\pi \in \mathcal{D}^{\text {hyp }}$ construct a new permutation $\tilde{\pi} \in \mathcal{D}\left(\pi_{n}\right)$ by adding the letter 1 to the left of the letter $n$ on the top and to the left of the letter 0 on the bottom. We will refer to the augmented hyperelliptic diagram. 


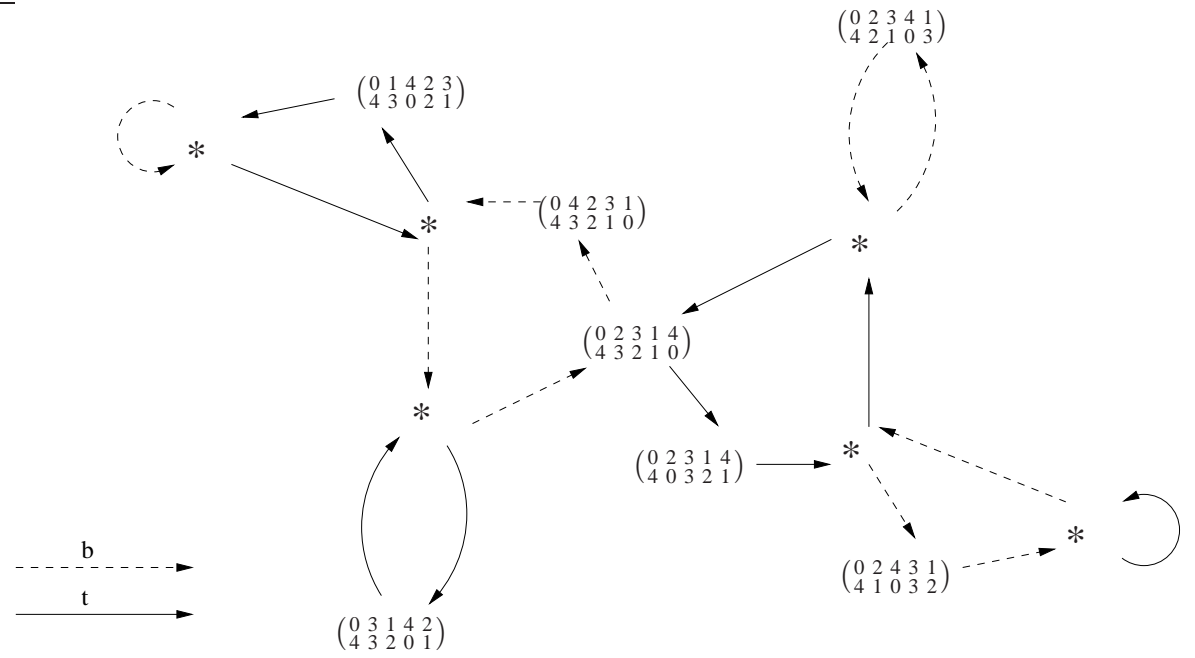

FIGURE 2. The augmented hyperelliptic diagram, and added permutations for $n=4$.

(2) Replace each edge $\pi \stackrel{k, n}{\longrightarrow} \pi^{\prime}$ in $\mathcal{D}^{h y p}$ by two edges $\tilde{\pi} \stackrel{k, n}{\longrightarrow} \pi^{\prime \prime} \stackrel{k, 1}{\longrightarrow} \tilde{\pi}^{\prime}$ in $\mathcal{D}\left(\pi_{n}\right)$, where the permutation $\pi^{\prime \prime}$ has the letter 1 for the last letter on the top.

(3) Replace each edge $\pi \stackrel{k, 0}{\longrightarrow} \pi^{\prime}$ in $\mathcal{D}^{h y p}$ by two edges $\tilde{\pi} \stackrel{k, 0}{\longrightarrow} \pi^{\prime \prime} \stackrel{k, 1}{\longrightarrow} \tilde{\pi}^{\prime}$ in $\mathcal{D}\left(\pi_{n}\right)$, where the permutation $\pi^{\prime \prime}$ has the letter 1 for the last letter on the bottom.

(4) We will denote by $\mathcal{A}_{n} \subset \mathcal{D}\left(\pi_{n}\right)$ the added permutations of the operations (2) and (3). All the edges are built except the cases when $\pi^{\prime \prime} \in \mathcal{A}_{n}$ and where 1 is winner.

Observe that operation (2) (respectively, (3)) arises exactly when the letter $n$ (respectively, 0 ) is looser in the diagram $\mathcal{D}^{\text {hyp }}$. Hence the description given in the previous section of this hyperelliptic diagram leads to the following.

Lemma 3.4. In $\mathcal{D}^{\text {hyp }}$, the edges where $n$ (respectively, 0) is looser are

$$
\mathcal{R}_{t}^{k}(\pi) \stackrel{*, n}{\longrightarrow} \mathcal{R}_{b} \mathcal{R}_{t}^{k}(\pi) \quad \text { (respectively, } \mathcal{R}_{b}^{k}(\pi) \stackrel{*, 0}{\longrightarrow} \mathcal{R}_{t} \mathcal{R}_{b}^{k}(\pi) \text { ), }
$$

for $k \in\{0, \ldots, n-2\}$. So, there are there are $n-1$ edges where $n$ is looser and $n-1$ edges where 0 is looser.

Therefore, the added permutations in $\mathcal{D}\left(\pi_{n}\right)$ by the operations (2) and (3) are:

$$
\begin{aligned}
& \mathcal{R}_{b} \mathcal{R}_{t}^{k}\left(\pi_{n}\right)=\left(\begin{array}{ccccccccccc}
0 & 2 & \ldots & k-1 & k & n & k+1 & \ldots & n-1 & 1 \\
n & k-1 & \ldots & 2 & 1 & 0 & n-1 & \ldots & k+1 & k
\end{array}\right), \\
& \mathcal{R}_{t} \mathcal{R}_{b}^{k}\left(\pi_{n}\right)=\left(\begin{array}{ccccccccc}
0 & n-k+2 & \ldots & n-1 & 1 & n & 2 & \ldots & n-k+1 \\
n & n-1 & \ldots & n-k+2 & n-k+1 & 0 & n-k & \ldots & 1
\end{array}\right),
\end{aligned}
$$

for $k \in\{2, \ldots, n-1\}$, and

$$
\begin{aligned}
& \mathcal{R}_{t}\left(\pi_{n}\right)=\left(\begin{array}{ccccccc}
0 & 2 & 3 & \ldots & n-1 & 1 & n \\
n & 0 & n-1 & \ldots & 3 & 2 & 1
\end{array}\right) \\
& \mathcal{R}_{b}\left(\pi_{n}\right)=\left(\begin{array}{ccccccc}
0 & n & 2 & 3 & \ldots & n-1 & 1 \\
n & n-1 & \ldots & 3 & 2 & 1 & 0
\end{array}\right)
\end{aligned}
$$


In addition for all $k=2, \ldots, n-1$ the two new permutations in the labeled diagram $\mathcal{D}\left(\pi_{n}\right)$ defined by (5) and (6) correspond to the same permutation in the reduced diagram $\mathcal{D}_{r}\left(\pi_{n}\right)$; the renumbering corresponds to $\sigma^{-k}$ with $\sigma$ the cyclic permutation $(1,2, \ldots, n-1)$. The two new permutations corresponding to $\mathcal{R}_{t}\left(\pi_{n}\right)$ and $\mathcal{R}_{b}\left(\pi_{n}\right)$ are different in $\mathcal{D}_{r}\left(\pi_{n}\right)$.

Proof of Lemma 3.4 The proof is obtained by straightforward computation and is left to the reader.

Now in order to finish the construction of $\mathcal{D}\left(\pi_{n}\right)$ and $\mathcal{D}_{r}\left(\pi_{n}\right)$, one has to consider from the added permutations $\mathcal{A}_{n}$ the operations top/bottom where the letter 1 is winner. In fact it turns out that the reduced diagram $\mathcal{D}_{r}\left(\pi_{n}\right)$ is already constructed. Namely,

Corollary 3.5. The diagram $\mathcal{D}_{r}\left(\pi_{n}\right)$ corresponds to adding the new permutations defined in Lemma 3.4 namely $\mathcal{T}_{n}$ to the augmented hyperelliptic diagram, up to renumbering. In particular the cardinality of this diagram is $2^{n-1}-1+n$.

Proof of Corollary 3.5. Let $\pi^{\prime} \in \mathcal{A}_{n}$ be an added permutation. Assume that it is given by (5), that is $\pi^{\prime}=\mathcal{R}_{b} \mathcal{R}_{t}^{k}\left(\pi_{n}\right)$ for some $k$. The edge $\pi^{\prime} \rightarrow \mathcal{R}_{b}\left(\pi^{\prime}\right)$ was constructed when defining $\mathcal{T}_{n}$. By Lemma 3.4 we also have that $\pi^{\prime}=\mathcal{R}_{t} \mathcal{R}_{b}^{k}\left(\pi_{n}\right)$ in the reduced RAUZY diagram, hence the edge $\pi^{\prime} \rightarrow \mathcal{R}_{t}\left(\pi^{\prime}\right)$ was also already constructed. This corresponds to $n-2$ added permutations.

The remaining cases are when $\pi^{\prime}$ is given by (7) or (8). Then is clear that the array corresponding to 1 being winner are arrays from $\pi^{\prime}$ to itself. This corresponds to 2 permutations.

Hence, the diagram $\mathcal{D}_{r}\left(\pi_{n}\right)$ is completely built. Since $\# \mathcal{D}^{h y p}=2^{n-1}-1$ one has $\# \mathcal{D}_{r}\left(\pi_{n}\right)=$ $2^{n-1}-1+n$.

Lemma 3.4 and Corollary 3.5 imply the following description of the diagram $\mathcal{D}\left(\pi_{n}\right)$.

Proposition 3.6. The augmented hyperelliptic diagram together with the permutations $\mathcal{T}_{n}$ form a fundamental domain for the covering map $\mathcal{D}\left(\pi_{n}\right) \longrightarrow \mathcal{D}_{r}\left(\pi_{n}\right)$. A fiber for this covering consists of labeled permutations of the kind $\left\{\pi, \pi \circ \sigma, \ldots, \pi \circ \sigma^{n-2}\right\}$, where $\pi \circ \sigma^{k}$ means the labeled permutation $\pi$ after renumbering with $\sigma^{k}$. In particular the cardinality of this diagram is $\left(2^{n}-1+n\right)(n-1)$.

The following technical lemma is similar to Corollary 3.3. It is a very important lemma since it will allow us to give information about all irreducible paths.

Lemma 3.7. Let $\gamma$ be a closed oriented path in $\mathcal{D}\left(\pi_{n}\right)$ : Let $k \in\{2, \ldots, n-2\}$ and let $k^{\prime} \in$ $\{2, n-k\}$ and let $\varepsilon \in\{t, b\}$.

a) If $\gamma$ contains the step $\mathcal{R}_{\varepsilon}^{k^{\prime}-1} \mathcal{R}_{1-\varepsilon}^{k}\left(\pi_{n}\right) \rightarrow \mathcal{R}_{\varepsilon}^{k^{\prime}} \mathcal{R}_{1-\varepsilon}^{k}\left(\pi_{n}\right)$, then it also contains the step $\mathcal{R}_{\varepsilon}^{k^{\prime}} \mathcal{R}_{4-\varepsilon}^{k}\left(\pi_{n}\right) \rightarrow \mathcal{R}_{\varepsilon}^{k^{\prime}+1} \mathcal{R}_{1-\varepsilon}^{k}\left(\pi_{n}\right)$.

b) If $\gamma$ contains the step $\mathcal{R}_{\varepsilon}^{k-1}\left(\pi_{n}\right) \rightarrow \mathcal{R}_{\varepsilon}^{k}\left(\pi_{n}\right)$, then it also contains the step $\mathcal{R}_{\varepsilon}^{k}\left(\pi_{n}\right) \rightarrow$ $\mathcal{R}_{\varepsilon}^{k+1}\left(\pi_{n}\right)$, or the step $\mathcal{R}_{-\varepsilon}^{k}\left(\pi_{n} \circ \sigma^{i}\right) \rightarrow \mathcal{R}_{1-\varepsilon}^{k+1}\left(\pi_{n} \circ \sigma^{i}\right)$, for some $i \in\{0, \ldots, n-2\}$. 


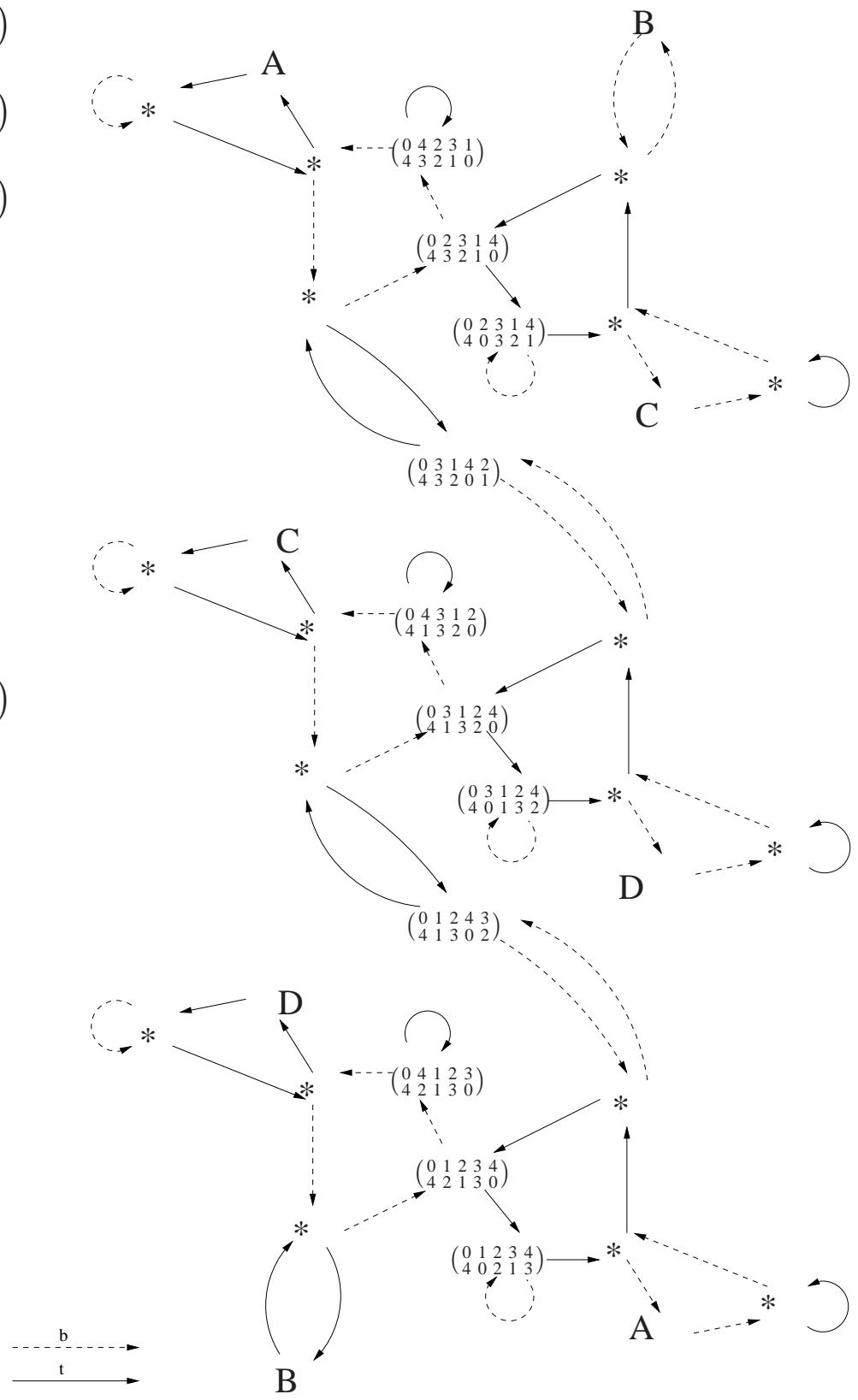

FIgURE 3. The complete diagram $\mathcal{D}\left(\pi_{n}\right)$ for $n=4$. The vertices labeled by letters $A, B, C$ and $D$ should be identified.

This property is also true if $\gamma$ is a nonclosed path that starts and ends in the set $\left\{\pi_{n}, \pi_{n} \circ \sigma, \ldots, \pi_{n} \circ\right.$ $\left.\sigma^{n-2}\right\}$. 
Proof. a) Using the construction of $\mathcal{D}\left(\pi_{n}\right)$ from $\mathcal{D}^{\text {hyp }}$, we see that, as in Corollary 3.3, the diagram $\mathcal{D}\left(\pi_{n}\right) \backslash\left\{\mathcal{R}_{\varepsilon}^{k^{\prime}} \mathcal{R}_{-\varepsilon}^{k}\left(\pi_{n}\right)\right\}$ is nonconnected.

b) Here, we have that $\mathcal{D}\left(\pi_{n}\right) \backslash\left\{\mathcal{R}_{\varepsilon}^{k}\left(\pi_{n}\right), \mathcal{R}_{1-\varepsilon}^{k}\left(\pi_{n} \circ \sigma^{i}\right)\right\}$ is nonconnected, for the parameter $i$ such that $\mathcal{R}_{1-\varepsilon} \mathcal{R}_{\varepsilon}^{k}\left(\pi_{n}\right)=\mathcal{R}_{\varepsilon} \mathcal{R}_{1-\varepsilon}^{k}\left(\pi_{n} \circ \sigma^{i}\right)$.

\section{Minimal Dilatations in A HYPERELliptic CONNECTED COMPONENT}

4.1. A key proposition. VEECH's construction can only build a pseudo-ANOSOV homeomorphism fixing a separatrix. But there can be many pseudo-ANOSOV homeomorphism that do not fix any separatrix [Los09, Lan10]. In our case, we are saved by the following key proposition.

Proposition 4.1. Let $g \geq 1$ and let $(X, \omega)$ be a flat surface in some hyperelliptic connected component $\mathcal{H}^{\text {hyp }}(2 g-2)$ or $\mathcal{H}^{\text {hyp }}(g-1, g-1)$. Then for any pseudo-ANOSOV homeomorphism $\phi$ affine with respect to $(X, \omega), \phi^{2}$ has a fixed point of negative index i.e. $\phi^{2}$ fixes a point and a outgoing separatrix issued from that point.

Proof of Proposition 4.1. Let $\tau$ be the hyperelliptic involution on $X$. Firstly by Proposition $2.2 \tau$ commutes with $\phi$. Thus $\phi$ descends to a pseudo-ANOSOV homeomorphism $f$ on the sphere. By assumption, $f$ has a single zero (of order $2 g-3$ or $2 g-2$ depending the case). Thus $f$ fixes the zero and induces a disc pseudo-ANOSOV homeomorphism, say $g$. Now by BROUWER's theorem, $g$ has a fixed point on the disc. Either this point is inside the disc (thus this is a regular point) or on the boundary. If the last case occur, then $f$ fixes the separatrices issued from the singularity. It is then not hard to see that either $\phi^{2}$ fixes a regular point and the outgoing separatrix, or a singular point and the outgoing separatrices. Proposition 4.1 is proven.

Remark 4.1. Another BROUWER's theorem states that a homeomorphism on the plane with a periodic orbit has a fixed point. With this theorem, it is easy to show that there actually exists a regular fixed point for $\phi^{2}$. However, we believe that the case where the separatrix is attached to a singularity is useful to present the main ideas without the technical difficulties of the other case.

The two next sections analyse the two cases depending wether the fixed point is singular or regular.

4.2. Case when the fixed separatrix is adjacent to the singularity. In this section, we study the case when the pseudo-ANOSOV homeomorphism fixes a horizontal separatrix starting from a singular point. We thus have to consider components $\mathcal{H}^{\text {hyp }}(2 g-2)$ and $\mathcal{H}^{\text {hyp }}(g-1, g-1)$. We first prove a sufficient condition for a primitive path to have a transition matrix with a spectral radius greater than 2 .

Proposition 4.2. Let $\gamma$ be a primitive closed path in some reduced RAUZY diagram. Let us assume that there is a lift $\hat{\gamma}$ of $\gamma$ in the labeled RAUZY diagram that contains all the letters as loosers, or all the letters as winners. Then the PERRON-FROBENIUS eigenvalue of the matrix $V(\gamma)$ is bounded from below by 2 . 
Proof of the proposition. If $\hat{\gamma}$ contains all the looser (respectively, all the winner) then Lemma 3.1 implies that the minimum of the sums of the columns (respectively, the lines) of the corresponding matrix $\widehat{V}$, before remuneration, is at least 2. Since $V(\gamma)=\widehat{V} \cdot P$ where $P$ is a permutation matrix (see Equation 2) the same property on the sums of the columns (respectively, the lines) holds for $V(\gamma)$.

By assumption, the matrix $V=V(\gamma)$ is primitive. Up to replacing $V$ by ${ }^{t} V$, which does not change its eigenvalues, we can assume that the sum of the coefficients on each lines is at least 2. Let $x$ be a PERRON-FROBENIUS eigenvector with positive entries associated to the PERRONFrobeniUs eigenvalue $\rho(V)$ of $V$. Let $i_{0}$ be such that $\min _{j=1, \ldots, d} x_{j}=x_{i_{0}}>0$. Then

$$
\rho(V) x_{i_{0}}=\sum_{j=1}^{d} v_{i_{0} j} x_{j} \geq x_{i_{0}} \sum_{j=1}^{d} v_{i_{0} j} .
$$

Since $x_{i_{0}}>0$, we see that there exists $i_{0}$ such that

$$
\rho(V) \geq \sum_{j=1}^{d} v_{i_{0} j} \geq 2 .
$$

Proposition 4.3. Let $\phi$ be a pseudo-ANOSOV homeomorphism affine on a translation surface $(X, \omega) \in \mathcal{H}^{\text {hyp }}(2 g-2)$ or $(X, \omega) \in \mathcal{H}^{\text {hyp }}(g-1, g-1)$. If $\phi$ fixes a horizontal separatrix emanating from a singular point of $\omega$ then the dilatation of $\phi$ is bounded from below by 2.

Proof. By VEECH's Theorem, $\phi$ is obtained by taking a closed loop in a RAUZY diagram. By a Lemma of RAUZY (see [Rau79]), any RAUZY diagram contains a vertex of the kind:

$$
v=\left(\begin{array}{lll}
\alpha & \ldots & \beta \\
\beta & \ldots & \alpha
\end{array}\right) .
$$

Since the underlying flat surface is in the hyperelliptic connected component, it is easy to see that $\mathrm{v}=\tau_{n}$ and therefore this RAUZY diagram is necessarily $\mathcal{D}^{\text {hyp }}$ (one can also use the main theorem of [Boi09]). Has we have seen, the labeled and reduced diagrams coincide. Hence, for any closed path in the reduced diagram whose associated matrix is primitive, the corresponding lift contains all the letters as winner. Hence by Proposition 4.2 the associated dilatation is at least 2.

4.3. Case when the fixed separatrix is adjacent to a regular point. One needs to prove the following proposition.

Proposition 4.4. Let $\phi$ be a pseudo-ANOSOV homeomorphism on a surface in $\mathcal{H}^{\text {hyp }}(2 g-2)$ or $\mathcal{H}^{\text {hyp }}(g-1, g-1)$. If $\phi$ fixes a regular point, then the dilatation of $\phi$ is bounded from below by 2 .

The idea of the proof is similar to the one of Proposition 4.3. we must consider pseudoANOSOV homeomorphisms that are obtained by the VEECH construction using the diagram 
$\mathcal{D}_{r}\left(\pi_{n}\right)$, were $\pi_{n}=\left(\begin{array}{cccccccc}0 & 2 & 3 & \ldots & n-1 & 1 & n \\ n & n-1 & \ldots & 3 & 2 & 1 & 0\end{array}\right)$. In this case, the reduced diagram is different from the labeled diagram (see section 3.7.2). But we will show that we can still apply Proposition4.2.

Remark 4.2. Our case is more subtle than the previous case. Indeed, start with the permutation $\left(\begin{array}{lllll}1 & 3 & 0 & 2 & 4 \\ 4 & 3 & 2 & 1 & 0\end{array}\right) \in \mathcal{D}\left(\pi_{4}\right)$. If we consider the path $b-t-b-t-t-t$ then the letter 3 is never winner nor looser. But the corresponding path in $\mathcal{D}_{r}\left(\pi_{4}\right)$ is closed and primitive. Nevertheless the lift based at the permutation $\left(\begin{array}{lllll}1 & 2 & 3 & 0 & 4 \\ 4 & 3 & 2 & 0 & 1\end{array}\right)$ is $t-t-t-b-t-b$, and all the letters are looser.

We will call the permutations in the diagram $\mathcal{D}\left(\pi_{n}\right)$ that are in $\left\{\pi_{n}, \pi_{n} \circ \sigma, \ldots, \pi_{n} \circ \sigma^{n-2}\right\}$ the central permutations. We will call permutations in the diagram $\mathcal{D}\left(\pi_{n}\right)$ that corresponds to $\mathcal{A}_{n}$ up to renumbering with $\sigma^{k}$ the transition permutations.

We have the following technical lemmas.

Lemma 4.5. Let $\gamma$ be a primitive closed path in $\mathcal{D}_{r}\left(\pi_{n}\right)$. There is a lift $\hat{\gamma}$ of $\gamma$ in $\mathcal{D}\left(\pi_{n}\right)$, not necessarily closed, that starts and ends at central permutations.

Lemma 4.6. Let $\hat{\gamma}$ be a path in $\mathcal{D}_{r}\left(\pi_{n}\right)$ that starts and ends at central permutations. Then $\hat{\gamma}$ contains all the letters $0,1,2, \ldots, n$ as looser.

We first prove the proposition assuming Lemma 4.5 and Lemma 4.6

Proof of Proposition 4.4 Let $\phi$ be a pseudo-ANOSOV homeomorphism that fixes a regular point. Then it also fixes its horizontal outgoing separatrix. By VEECH's theorem $\phi$ is obtain by taking a closed loop in some RAUZY diagram. Using RAUZY Lemma stated in the proof of Proposition 4.3, we can show that the RAUZY diagram is necessarily $\mathcal{D}_{r}\left(\pi_{n}\right)$ (one can also use the main theorem of [Boi09]). Let $\gamma$ be the corresponding closed primitive path in $\mathcal{D}_{r}\left(\pi_{n}\right)$. By Lemma4.5, there is a lift of $\gamma$ that starts and ends at central permutations. This lift contains all the letters as loosers by Lemma 4.6, and by Proposition 4.2, the PERRON-FrOBENIUS eigenvalue of the corresponding matrix, and hence the dilatation of $\phi$ is at least 2.

The proof of the lemmas is strongly related to the geometry of the diagram $\mathcal{D}\left(\pi_{n}\right)$. Before giving a formal proof, we first present a very informal proof that uses the informal description of the diagram given in the beginning of Section 3.7 .2

(1) For the first lemma it is enough to prove that a primitive closed path in the labeled RAUZY diagram must pass through a central permutation. Such path must pass through a principal loop were 0 or $\mathrm{n}$ is winner. If it enters in a k-th secondary loop (attached to the k-th vertex of a principal loop), the geometry of the diagram imposes that it either leaves the secondary loop from the same vertex (and therefore joins the $(\mathrm{k}+1)$-th vertex of the principal loop) or joins a k-th secondary loop in another leaf and escape it at the k-th vertex of another principal loop, and therefore joins the $(\mathrm{k}+1)$-th vertex of this loop. Iterating this argument, it eventually joins a central permutation. 
(2) The key observation for the second lemma is that a path joining two central permutations will either pass through all the vertices of a principal loop, or will pass from one principal loop to another one through a transition permutation and the corresponding pair of secondary loops. In any cases all the letters will appear as loosers.

We now give a proof of the lemmas.

Proof of Lemma 4.5 Let $\gamma$ be a primitive closed path in $\mathcal{D}_{r}\left(\pi_{n}\right)$, and let $\hat{\gamma}$ be a lift of $\gamma$ in $\mathcal{D}\left(\pi_{n}\right)$. The path $\hat{\gamma}$ is not necessarily closed, but a power of $\gamma$ admits a lift $\eta$ which is closed and primitive. Furthermore, $\eta$ consists of a concatenation of lifts of $\gamma$. By Proposition 3.2 the path $\eta$ contains all the letter $0, \ldots, n$ as winner, in particular the letter 0 as winner.

The point is that the letter 0 appears as winner only on the steps of the form $\mathcal{R}_{b}^{k-1}\left(\pi_{n} \circ \sigma^{i}\right) \rightarrow$ $\mathcal{R}_{b}^{k}\left(\pi_{n} \circ \sigma^{i}\right)$. One can assume that $i=0$. By Lemma 3.7 there exists in $\eta$ the step $\mathcal{R}_{b}^{k}\left(\pi_{n}\right) \stackrel{0, *}{\longrightarrow}$ $\mathcal{R}_{b}^{k+1}\left(\pi_{n}\right)$ or the step $\mathcal{R}_{t}^{k}\left(\pi_{n} \circ \sigma^{j}\right) \stackrel{n, *}{\longrightarrow} \mathcal{R}_{t}^{k+1}\left(\pi_{n} \circ \sigma^{j}\right)$, where $j$ corresponds to the index such that $\mathcal{R}_{b} \mathcal{R}_{t}^{k}\left(\pi_{n} \circ \sigma^{j}\right)=\mathcal{R}_{t} \mathcal{R}_{b}^{k}\left(\pi_{n}\right)$.

Since, 0 and $n$ play a symmetric role, we can iterate the argument and therefore, we must reach $k=n$, which corresponds to a central permutation. Hence $\eta$ contains a central permutation. Since $\eta$ is a concatenation of lifts of $\gamma$, this path passes through the reduced permutation $\pi_{n}$. Since $\gamma$ is closed, we can assume that it starts and ends at $\pi_{n}$. Hence, any lift of $\gamma$ has endpoints that are in the preimage of $\pi_{n}$, which are the central permutations. Lemma 4.5 is proven.

Proof of Lemma 4.6. Let $\hat{\gamma}$ be a path in $\mathcal{D}\left(\pi_{n}\right)$ connecting two central permutations. For simplicity, assume that $\hat{\gamma}(1)$ is $\pi_{n}$. Again, by symmetry, one can assume that the first arrow is given by the map $\mathcal{R}_{t}$, i.e. $\hat{\gamma}(1) \stackrel{n, 0}{\rightarrow} \hat{\gamma}(2)=\mathcal{R}_{t}\left(\pi_{n}\right)$. By Lemma 3.7 there exists $i_{2}, \ldots, i_{n-1}$ such that the path $\hat{\gamma}$ contains the steps $\mathcal{R}_{\varepsilon_{2}}^{2}\left(\pi_{n} \circ \sigma^{i_{2}}\right) \rightarrow \mathcal{R}_{\varepsilon_{2}}^{3}\left(\pi_{n} \circ \sigma^{i_{2}}\right), \ldots, \mathcal{R}_{\varepsilon_{n-1}}^{n-1}\left(\pi_{n} \circ \sigma^{i_{n-1}}\right) \rightarrow \mathcal{R}_{\varepsilon_{n-1}}^{n}\left(\pi_{n} \circ \sigma^{i_{n}}\right)$.

The first possibility is that $\hat{\gamma}$ does not change of leaf, i.e. for all $k \in\{2, \ldots, n-1\}, i_{k}=0$. Then the steps previously written form a subpath that can be rewritten as $\hat{\gamma}(1) \stackrel{n, 0}{\longrightarrow} \hat{\gamma}\left(j_{2}\right) \stackrel{n, 1}{\longrightarrow} \hat{\gamma}\left(j_{3}\right) \stackrel{n, 2}{\longrightarrow}$ $\ldots \stackrel{n, n-1}{\longrightarrow} \hat{\gamma}(1)$. Then all the letters $0, \ldots, n-1$ are loosers. The path $\hat{\gamma}$ is closed, so $n$ cannot always be winner. Hence it is also looser.

The second possibility is that $\hat{\gamma}$ changes of leaf, i.e. there exists a smallest $k$ such that $i_{k}>0$. Then by Lemma 3.7, a subpath of $\gamma$ is obtained by the following way (compare with Figure 3):

- We start from $\pi_{n}$.

- We apply $k$ times the RAUZY move $\mathcal{R}_{t}$ (these are the moves $\hat{\gamma}(1) \stackrel{n, 0}{\longrightarrow} \hat{\gamma}\left(j_{2}\right) \stackrel{n, 1}{\longrightarrow} \ldots \stackrel{n, k}{\longrightarrow}$ $\left.\hat{\gamma}\left(j_{k+1}\right)=\mathcal{R}_{t}^{k}\left(\pi_{n}\right)\right)$.

- We apply 1 time the RAUZY move $\mathcal{R}_{b}$ (we reach the permutation $\mathcal{R}_{b} \mathcal{R}_{t}^{k}\left(\pi_{n}\right)=\mathcal{R}_{t} \mathcal{R}_{b}^{k}\left(\pi_{n} \circ\right.$ $\left.\sigma^{i_{k}}\right)$ ). This is the move $\gamma\left(j_{k+1}\right) \stackrel{k, n}{\longrightarrow} \hat{\gamma}\left(j_{k+2}\right)$.

- We apply $k^{\prime}=n-k$ times the RAUZY move $\mathcal{R}_{t}$ (until we get the permutation $\mathcal{R}_{b}^{k}\left(\pi_{n} \circ\right.$ $\left.\sigma^{i_{k}}\right)$ ). These are the moves $\hat{\gamma}\left(j_{k+2}\right) \stackrel{1, k}{\longrightarrow} \hat{\gamma}\left(j_{k+3}\right) \stackrel{1, k+1}{\longrightarrow} \ldots \stackrel{1, n-1}{\longrightarrow} \hat{\gamma}\left(j_{n+2}\right)$. 
We see that all the letters are loosers. This proves Lemma 4.6.

\section{APPENDIX A. EXAMPLES IN HYPERELLIPTIC COMPONENTS}

In this appendix we show that the uniform lower bound on dilatations of pseudo-ANOSOV homeomorphisms in Theorem 1.1 is sharp by constructing suitable examples. This will thus give a proof of Theorem 1.2 .

\section{A.1. Hyperelliptic connected component $\mathcal{H}^{\text {hyp }}(2 g-2)$.}

Proposition A.1. Let $g \geq 2$. There exists a pseudo-ANOSOV homeomorphism $\phi_{g}$ affine on a translation surface in $\mathcal{H}^{\text {hyp }}(2 g-2)$ whose dilatation is the PERRON root of the polynomial $X^{2 g+1}-2 X^{2 g-1}-2 X^{2}+1$. This dilatation satisfies

$$
0<\theta\left(\phi_{g}\right)-\sqrt{2}<\frac{1}{2^{2 g-3}} .
$$

Lemma A.2. The polynomial $P_{g}=X^{2 g+1}-2 X^{2 g-1}-2 X^{2}+1$ admits a unique real root $\alpha$ greater than $\sqrt{2}$.

Proof. $P_{g}(\sqrt{2})=-4+1<0$, and $P_{g}(x)>0$ for $x$ large enough and $P_{g}^{\prime}(x)>0$ for $x>\sqrt{2}$.

Lemma A.3. Let $M=\left[m_{i j}\right]$ be the matrix of dimension $2 g$ defined by:

- for all $j \in\{g+1, \ldots, 2 g\}, m_{1, j}=1$, and $m_{1, g}=2$,

- for all $i \in\{2, \ldots, g\}, m_{i, i+g-1}=1$,

- for all $j \in\{g+1, \ldots, 2 g-1\}, m_{i, i-g}=1$,

- $m_{2 g, g}=m_{2 g, 2 g}=1$.

- all the other elements are zero.

$$
M_{g}=\left(\begin{array}{c|ccccc|c} 
& 2 & 1 & \cdots & \cdots & 1 & 1 \\
& 0 & 1 & 0 & \cdots & 0 & 0 \\
0_{g \times g-1} & \vdots & \ddots & \ddots & \ddots & \vdots & \vdots \\
& \vdots & & \ddots & \ddots & 0 & \vdots \\
& 0 & \cdots & \cdots & 0 & 1 & 0 \\
\hline & & & & & & 0 \\
I_{g-1 \times g-1} & & & & & \\
& & & & & & \vdots \\
& & & & & & 0 \\
\hline 0 & 1 & 0 & \cdots & \cdots & 0 & 1
\end{array}\right)
$$

Then $M_{g}$ contains $\alpha$ and $1 / \alpha$ as eigenvalues.

Proof. We must compute the characteristic polynomial $\chi_{g}=\operatorname{det}\left(M_{g}-x I_{2 g \times 2 g}\right)$. Since $P_{g}$ is reciprocal and $\alpha \neq-1$, we just need to show that $(X+1) \chi_{g}=P_{g}$ for all $g \geq 2$. We denote by $L_{i}$ 
the $i-t h$ line. For each $i \in\{1, g\}$ we replace $L_{i}$ by $L_{i}+x L_{i+g}$. Then we develop the determinant $g$ times along the first column, to get:

$$
\begin{aligned}
\operatorname{det}\left(M_{g}-x I\right) & =(-1)^{(g+2)(g-1)}\left(2(1-x)+(-1)^{g+2}\left((-1)^{g+1} .1+x(1-x) D_{g-1}\right)\right), \\
& =2(1-x)+(-1)+(-1)^{g} x(1-x) D_{g-1},
\end{aligned}
$$

were $D_{g-1}$ is the $(g-1) \times(g-1)$ determinant:

$$
D_{g-1}=\left|\begin{array}{cccccc}
1-x^{2} & 1 & 1 & \ldots & \ldots & 1 \\
1 & -x^{2} & 0 & \ldots & \ldots & 0 \\
0 & 1 & -x^{2} & 0 & \ldots & 0 \\
\vdots & \ddots & \ddots & \ddots & \ddots & \vdots \\
\vdots & & 0 & 1 & -x^{2} & 0 \\
0 & \ldots & \ldots & 0 & 1 & -x^{2}
\end{array}\right| .
$$

Developing $D_{g}$ on the last column, we obtain $D_{g}=-x^{2} D_{g-1}+(-1)^{g+1}$. Then using this last expression, we see that:

$$
(X+1)\left(\chi_{g+1}-X^{2} \chi_{g}\right)=(X+1)\left(1-X-2 X^{2}+2 X^{3}\right)=\left(2 X^{4}-3 X^{2}+1\right)=P_{g+1}-X^{2} P_{g}
$$

Since $(1+X) \chi_{2}=P_{2}$, we deduce that $(1+X) \chi_{g}=P_{g}$ for all $g \geq 2$, which proves the proposition.

Lemma A.4. Let $\lambda$ (respectively, $\tau$ ) be an eigenvector of $M$ for the eigenvalue $\alpha$ (respectively, $1 / \alpha)$. Then, up to replacing $\lambda$ or $\tau$ by its opposite, $(\lambda, \tau)$ defines a suspension datum for the permutations $\pi$ and $\pi^{\prime}$, with:

$$
\pi=\left(\begin{array}{ccccccc}
1 & 2 & \ldots & \ldots & \ldots & 2 g-1 & 2 g \\
2 g & g & \ldots & 1 & 2 g-1 & \ldots & g+1
\end{array}\right) \quad \text { and } \quad \pi^{\prime}=\left(\begin{array}{cccccccc}
g+1 & \ldots & \ldots & 2 g-1 & 1 & \ldots & g & 2 g \\
2 g & 2 g-1 & \ldots & \ldots & \ldots & \ldots & 2 & 1
\end{array}\right)
$$

Moreover $(\pi, \lambda, \tau)$ and $\left(\pi^{\prime}, \lambda, \tau\right)$ define two isometric surfaces.

Proof. We have $M_{g} \lambda=\alpha \lambda$ and $M_{g} \tau=\frac{1}{\alpha} \tau$. This gives:

$$
\begin{array}{rlrl}
\forall i \in\{1, \ldots, 2 g-2\} \backslash\{g\} & \lambda_{i}=\alpha^{2} \lambda_{i+1} & \text { and } & \tau_{i}=\frac{1}{\alpha^{2}} \tau_{i+1} \\
\lambda_{2 g-1}=\alpha \lambda_{g} & \text { and } & \tau_{2 g-1}=\frac{1}{\alpha} \tau_{g} \\
\lambda_{g}+\lambda_{2 g}=\alpha \lambda_{2 g} & \text { and } & \tau_{g}+\tau_{2 g}=\frac{1}{\alpha} \tau_{2 g}
\end{array}
$$

Since $\alpha>1$, Equation (11) implies that $\lambda_{g}$ is of the same sign as $\lambda_{2 g}$. The other equations imply that all the $\lambda_{i}$ are of the same sign. Hence all the $\lambda_{i}$ are positive if we choose $\lambda_{2 g}>0$.

By a similar argument, for $\tau$, we see that we can choose $\tau_{2 g}<0$ and have $\tau_{i}>0$ for all $i<2 g$. Furthermore, the label " $2 \mathrm{~g}$ " is the last of the first line of $\pi$ (respectively, $\pi^{\prime}$ ) and the first of the 
second line of $\pi$ (respectively, $\pi^{\prime}$ ). Then in order to prove that $(\lambda, \tau)$ is a suspension data for $\pi$ and $\pi^{\prime}$, it is enough to show that $s=\sum_{i=1}^{2 g} \tau_{i}$ is negative. By Equations (9) and (10), we have

$$
s=\tau_{2 g}+\sum_{k=0}^{2 g-2} \frac{1}{\alpha^{k}} \tau_{g}=\frac{1}{\alpha} \tau_{2 g}+\sum_{k=1}^{2 g-2} \frac{1}{\alpha^{k}} \tau_{g}=\ldots=\frac{1}{\alpha^{2 g-1}} \tau_{2 g}<0 .
$$

Hence we have proven that $(\pi, \lambda, \tau)$ and $\left(\pi^{\prime}, \lambda, \tau\right)$ define translation surfaces by the VEECH's construction (see Section 3.2). Now we remark that, if $P_{1}$ is the polygon associated to $(\pi, \lambda, \tau)$, and $P_{2}$ is the polygon associated to $\left(\pi^{\prime}, \lambda, \tau\right)$, then we obtain $P_{2}$ from $P_{1}$ by a $180^{\circ}$ rotation. Since these flat surfaces are hyperelliptic we conclude that the two surfaces defined by $(\pi, \lambda, \tau)$ and $\left(\pi^{\prime}, \lambda, \tau\right)$ are isometric.

Lemma A.5. There is a pseudo-ANOSOV homeomorphism on the surface defined by the data $(\pi, \lambda, \tau)$ with dilatation $\alpha$.

Proof. We start from $\pi^{\prime}$, and consider the path $\gamma$ in the corresponding RAUZY graph obtained by applying to $\pi$ map $\mathcal{R}_{b} g$ times and then one time the map $\mathcal{R}_{t}$. We obtain the permutation

$$
\pi^{\prime \prime}=\left(\begin{array}{cccccccc}
g+1 & \ldots & \ldots & 2 g-1 & 1 & \ldots & g & 2 g \\
2 g & 1 & 2 g-1 & \ldots & \ldots & \ldots & \ldots & 2
\end{array}\right)
$$

The sequence of winners/loosers is $(1,2 g),(1, g), \ldots,(1,2),(2 g, 1)$. And therefore, the corresponding transition matrix is:

$$
M_{g}^{\prime}=\left(\begin{array}{ccccc|c|c}
2 & 1 & \cdots & \cdots & 1 & & 1 \\
0 & 1 & 0 & \cdots & 0 & & 0 \\
\vdots & \ddots & \ddots & \ddots & \vdots & 0_{g \times g-1} & \vdots \\
\vdots & & \ddots & \ddots & 0 & & \vdots \\
0 & \cdots & \cdots & 0 & 1 & & 0 \\
\hline & & & & & & 0 \\
& & & & & \\
& & & & \\
& & & & & & \vdots \\
& & & & & & 0 \\
\hline 1 & 0 & \cdots & \cdots & 0 & 0 & 1
\end{array}\right)
$$

The permutation $\pi^{\prime \prime}$ is obtained from $\pi$ after renumbering. Multiplying $M^{\prime}$ by the corresponding permutation matrix, one get precisely the matrix $M_{g}$. Therefore, if we consider $\lambda^{\prime \prime}=\frac{1}{\alpha} \lambda$, renumber it and do backward the path $\gamma$, one get $\left(\pi^{\prime}, \lambda\right)$. Therefore, if we start from $(\pi, \lambda, \tau)$, then consider $\left(\pi^{\prime}, \lambda, \tau\right)$, and then apply the RAUZY induction, and renumbering, one will obtain $\left(\pi, \frac{1}{\alpha} \lambda, \alpha \tau\right)$. Each time, we obtain new parameter for the same flat surface, hence $(\pi, \lambda, \tau)$ and $\left(\pi, \frac{1}{\alpha}, \lambda, \alpha \tau\right)$ define the same element in the moduli space. So the corresponding flat surface admit a pseuso-Anosov homeomorphism with dilatation $\alpha$. 
Proof of the proposition. The existence of the pseudo-ANOSOV homeomorphism $\phi_{g}$ is clear from the previous lemmas. We have $\alpha=\theta\left(\phi_{g}\right)$, and:

$$
\alpha^{2 g+1}-2 \alpha^{2 g-1}-2 \alpha^{2}+1=\alpha^{2}\left(\alpha^{2 g-1}-2 \alpha^{2 g-3}-2\right)+1=0,
$$

thus

$$
\alpha^{2 g-1}-2 \alpha^{2 g-3}-2=\alpha^{2 g-3}\left(\alpha^{2}-2\right)-2<0 .
$$

Since $\alpha>\sqrt{2}$ we obtain $0<\alpha-\sqrt{2}<\frac{1}{2^{2 g-3}}$.

\section{A.2. Hyperelliptic connected component $\mathcal{H}^{\text {hyp }}(g-1, g-1)$.}

Proposition A.6. Let $g \geq 2$. There exists a pseudo-ANOSOV homeomorphism on a translation surface in $\mathcal{H}^{\text {hyp }}(g-1, g-1)$ with dilatation the PERRON root of the polynomial:

$$
\begin{array}{ll}
X^{2 g+2}-2 X^{2 g}-2 X^{g+1}-2 X^{2}+1, & \text { if } g \text { is even, } \\
X^{2 g+2}-2 X^{2 g}-4 X^{g+2}+4 X^{g}+2 X^{2}-1, & \text { if } g \text { is odd. }
\end{array}
$$

The dilatation satisfies

$$
0<\theta\left(\phi_{g}\right)-\sqrt{2}<\frac{4}{\sqrt{2}^{g}}
$$

Proof. The idea of the proof is very similar to the previous one. We just present here the corresponding paths in the RAUZY diagram.

- If $g$ is even, we start from the permutation $\pi=\left(\begin{array}{ccccccc}1 & \ldots & g+1 & g+2 & \ldots & 2 g & 2 g+1 \\ 2 g+1 & g+1 & \ldots & 1 & 2 g & \ldots & g+2\end{array}\right)$. As previously, we consider the "reverse" permutation $\pi^{\prime}=\left(\begin{array}{ccccccc}g+2 & \ldots & 2 g & 1 & \ldots & g+1 & 2 g+1 \\ 2 g+1 & 2 g & \ldots & g+2 & g+1 & \ldots & 1\end{array}\right)$. Then the path in the RAUZY diagram starting from $\pi^{\prime}$ and defined by $(g+1)$ times $b$, two times $t$. One gets the permutation $\pi^{\prime \prime}=\left(\begin{array}{ccccccccc}g+2 & \ldots & 2 g & 1 & & 2 & 3 \ldots & g+1 & 2 g+1 \\ 2 g+1 & 1 & 2 & 2 g & \ldots & g+2 & g+1 & \ldots & 3\end{array}\right)$, which is a renumbering of $\pi$, and the associated matrix is:

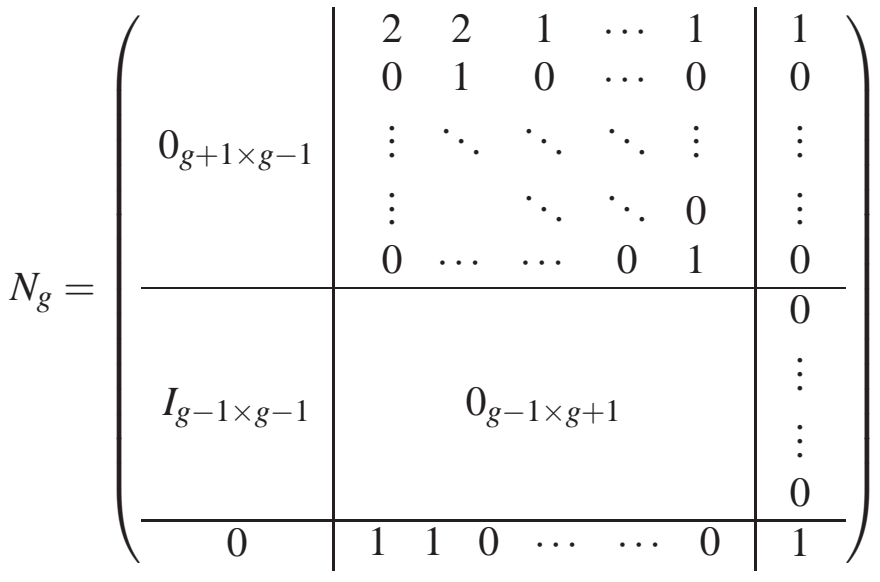


One can check that $(X+1) \chi_{N_{g}}(X)=X^{2 g+2}-2 X^{2 g}-2 X^{g+1}-2 X^{2}+1$, and that, for $\alpha$ the PERRON root of $\chi_{N_{g}}(X)$, the eigenvectors of $N_{g}$ corresponding to $\alpha$ and $\frac{1}{\alpha}$ define a suspension data for $\pi$ (and $\pi^{\prime}$ ). Then the corresponding translation surface admits the required pseudo-ANOSOV homeomorphism by construction.

Also, we have

$$
\alpha^{2 g}\left(\alpha^{2}-2\right)<2 \alpha^{g+1}+2 \alpha^{2}
$$

Thus,

$$
\alpha-\sqrt{2}<\frac{2}{\alpha+\sqrt{2}}\left(\frac{1}{\alpha^{g-1}}+\frac{1}{\alpha^{2 g-2}}\right)<\frac{2}{\sqrt{2}^{g}},
$$

since $\alpha>\sqrt{2}$.

- If $g$ is odd, we consider $\pi$ as previously and we take the path defined by $g$ times $b$, then $t-b-t-t$. We obtain the required pseudo-ANOSOV with the same construction we presented have above. Also, we have:

$$
\alpha-\sqrt{2}=\frac{1}{\alpha+\sqrt{2}} \frac{4 \alpha^{g}\left(\alpha^{2}-1\right)+1-2 \alpha^{2}}{\alpha^{2 g}}<\frac{4}{\alpha^{g}}<\frac{4}{\sqrt{2}^{g}}
$$

\section{APPENDIX B. EXAMPLES IN NON HYPERELLIPTIC COMPONENTS}

In this appendix we motivate assumptions of Theorem 1.1. We show that if we relax the condition on the hyperelliptic involution, one can construct pseudo-ANOSOV homeomorphisms in the non hyperelliptic connected components whose dilatations tend to 1 when the genus tends to infinity. This will thus give a proof of Theorem 1.3 .

We will construct a sequence of pseudo-ANOSOV homeomorphisms $\varphi_{g}$ on the flat sphere i.e. $\varphi_{g}$ is affine with respect to a quadratic differential $q_{g}$ on the sphere. The construction involves the RAUZY-VEECH induction for quadratic differentials [BL08], using generalized permutations.

Proposition B.1. For each $g \geq 3$, there exists a pseudo-ANOSOV homeomorphism $\varphi_{g}$ on the flat sphere with $2 g$ poles and two zeroes of order $g-2$, fixing a pole, and having for dilatations $\theta\left(\varphi_{g}\right)$ the PERRON root of the polynomial

$$
P=X^{2 g}-X^{2 g-1}-4 X^{g}-X+1 .
$$

Proof of the proposition. We consider the RAUZY diagram corresponding to the stratum of quadratic differentials on the sphere having the given singularity data. It is sufficient to give a closed path in this diagram and to check that the renormalisation matrix is irreducible and has $\theta_{g}$ for eigenvalue, where $\theta_{g}$ is the PERRON root of $P$.

Let us consider the following generalized permutation (on $2 g+1$ letters)

$$
\left(\begin{array}{cccccccc}
1 & 2 & 2 & 3 & 3 & \ldots & g+1 & g+1 \\
g+2 & g+3 & g+3 & \ldots & 2 g+1 & 2 g+1 & g+2 & 1
\end{array}\right) .
$$


For instance for $g=3$ this gives

$$
\left(\begin{array}{lllllll}
1 & 2 & 2 & 3 & 3 & 4 & 4 \\
5 & 6 & 6 & 7 & 7 & 5 & 1
\end{array}\right) .
$$

The RAUZY path we will consider is $t-b-t-b-t-b$. A simple calculation shows that the renormalization matrix $\widehat{V}$ (for the labeled permutation) and permutation matrix $P$ are, respectively

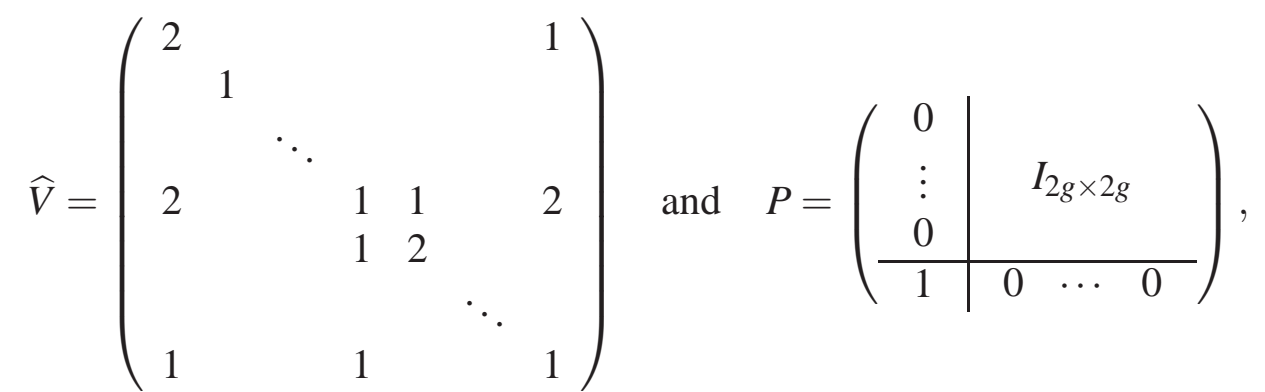

where the " 2 " in the diagonal of the matrix $\widehat{V}$ appears at the position $g+2$. All the other entries in $\widehat{V}$ are zeroes. Thus the renormalization matrix (for the reduced permutations) is $V=\widehat{V} \cdot P$. One can show that the matrix $V$ is irreducible and its characteristic polynomial is

$$
(X-1)\left(X^{2 g}-X^{2 g-1}-4 X^{g}-X+1\right)=(X-1) P(X) .
$$

Then as in the previous section, we see that some eigenvectors of $V$ define suspension data, and the corresponding flat surface has a pseudo-ANOSOV homeomorphism whose dilatation is the PERRON root of $P$.

Finally we have the following corollary, which justifies the assumptions of Theorem 1.1 on the hyperelliptic involution

Corollary B.2. For $g \geq 3$ odd, there exists an affine pseudo-ANOSOV homeomorphism $\phi_{g}$ on a translation surface $(X, \omega)$ with the following properties:

(1) $(X, \omega) \in \mathcal{H}^{\text {odd }}(g-1, g-1)$,

(2) $(X, \omega)$ is hyperelliptic,

(3) $\phi_{g}$ fixes a separatrix on $(X, \omega)$ (issued from a WEIERSTRASS point),

(4) the dilatation of $\phi_{g}$ is the PERRON root of the polynomial

$$
X^{2 g}-X^{2 g-1}-4 X^{g}-X+1 .
$$

In particular

$$
\lim _{g \rightarrow+\infty} \theta\left(\phi_{g}\right)=1
$$

Remark B.1. Obviously the hyperelliptic involution on $X$ fixes the two zeroes of $\omega$, and $(X, \omega)$ is not in the hyperelliptic connected component of the corresponding stratum. 
Proof of the corollary. For $g$ odd, the examples given by Proposition B.1 lift to pseudo-AnOSOV homeomorphisms $\phi_{g}$ on the orientating cover $(X, \omega) \in \mathcal{H}(g-1, g-1)$. By construction the surface is hyperelliptic and since the poles are ramification points, there is one lift that fixe a regular (WEIERSTRASS) point and the separatrix issued from that point.

To identify the connected component, we use the formula in [Lan04bis]. Since $(X, \omega) \rightarrow$ $\left(\mathbb{P}^{1}, q\right)$ is the orientating cover, the spin structure determined by $(X, \omega)$ is

$$
\left[\frac{\left|n_{+1}-n_{-1}\right|}{4}\right] \quad \bmod 2
$$

where $n_{+1}$ is the number of singularities of $q$ of degrees $1 \bmod 4$ and $n_{-1}$ is the number of singularities of $q$ of degrees $-1 \bmod 4$.

(1) If $g=1 \bmod 4$ : then $g-2=-1$ modulo 4, thus $n_{+1}=0$ and $n_{-1}=2 g+2$. Hence the parity of the spin structure on $(X, \omega)$ is (with $g=1+4 k$ )

$$
\left[\frac{\left|n_{+1}-n_{-1}\right|}{4}\right]=\frac{2 g+2}{4}=1+2 k=1 \bmod 2 .
$$

(2) If $g=-1 \bmod 4$ : then $g-2=1$ modulo 4, thus $n_{+1}=2$ and $n_{-1}=2 g$. Hence the parity of the spin structure on $(X, \omega)$ is (with $g=-1+4 k$ )

$$
\left[\frac{\left|n_{+1}-n_{-1}\right|}{4}\right]=\frac{2 g-2}{4}=-1+2 k=1 \bmod 2 .
$$

\section{REFERENCES}

[AY81] P. ARnOUX and J. C. YOCCOZ - "Construction de difféomorphismes pseudo-AnOsOv (French)", C. R. Acad. Sci. Paris Sér. I Math. 292 (1981), pp. 75-78.

[AGY06] A. Avila, S. Goü̈zEl and J.-C. YoCCOZ - "Exponential mixing for the TeiCHMÜLlER flow", Publ. Math. Inst. Hautes Études Sci. No. 104 (2006), pp. 143-211.

[AV07] A. AVILA and M. VIANA - "Simplicity of LYAPUNOV spectra: proof of the ZORICH-KONTSEVICH conjecture", Acta Math. 198 No. 1 (2007), pp. 1-56.

[BL08] C. BOISSY and E. LANNEAU - "Dynamics and geometry of the RAUZY-VEECH induction for quadratic differentials", Ergodic Theory Dynam. Systems 29 (2009), no. 3, pp. 767-816.

[Boi09] C. BoISSY - "Classification of RAUZY classes in the moduli space of quadratic differentials.", preprint arXiv:0904.3826 (2009).

[Boi10] _Labeled and reduced RAUZY classes", in preparation.

[CH08] J. CHO and J. HAM - "The minimal dilatation of a genus two surface", Experiment. Math. 17 (2008), pp. 257-267.

[Del10] V. DelecroiX - “Cardinality of RAUZY classes ”, preprint (2010).

[Far06] B. FARB - "Some problems on mapping class groups and moduli space", Problems on Mapping Class Groups and Related Topic ed. by B. FARB, Proc. Symp. Pure and Applied Math. 74, (2006) pp. 11-55.

[FLM08] B. FARB, C. LEININGER and D. MARGALIT - "The lower central series and pseudo-ANOSOV dilatations", American Journal of Mathematics 130 (2008), pp. 799-827. 
[FK92] H.M. FARKA and I. KRA - "RIEMANn surfaces, 2nd ed.", Grad. Text Math. 71 Springer-Verlag, Berlin, (1992).

[Fat87] A. FATHI - "Dehn twists and pseudo-AnOsov diffeomorphisms", Invent. math. 87 pp. 129-151 (1987).

[FLP79] A. FAthi, F. LAudenbach and V. PoÉnaru - "Travaux de Thurston sur les surfaces", Astérisque 66-67 (1979).

[HK06] E. HIRONAKA and E. KIN - "A family of pseudo-ANOSOv braids with small dilatation", Algebraic \& Geometric Topology 6 (2006) , pp. 699-738.

[Hir09] E. HIRONAKA - "Small dilatation pseudo-Anosov mapping classes coming from the simplest hyperbolic braid", preprint arXiv:0909.4517 (2009).

[Iva88] N.V. IVANOV - "Coefficients of expansion of pseudo-ANOSOV homeomorphisms", Zap. Nauchn. Sem. Leningrad. Otdel. Mat. Inst. Steklov (LOMI) 167 (1988), translation in J. Soviet Math. 52 (1990), pp. 2819-2822.

[KZ03] M. KontSEvich and A. ZORICH - "Connected components of the moduli spaces of Abelian differentials with prescribed singularities", Invent. Math. 153 (2003), no. 3, pp. 631-678.

[KT10] E. KIN and M. TAKASAWA - "Pseudo-Anosovs on closed surfaces having small entropy and the Whitehead sister link exterior", preprint [ps] arXiv:1003.0545(2010).

[FL87] J. Fehrenbach and J. Los - "Une minoration de l'entropie topologique des difféomorphismes du disque (French) ", J. London Math. Soc. 60 (1999), no. 3, 912-924.

[Lan04] E. LANNEAU - "Hyperelliptic components of the moduli spaces of quadratic differentials with prescribed singularities", Comment. Math. Helv. 79 (2004), pp. 471-501.

[Lan04bis] __, Parity of the spin structure defined by a quadratic differential, Geom. Topol. 8 (2004) 511-538 (electronic).

[Lan08] _ _Connected components of the strata of the moduli spaces of quadratic differentials with prescribed singularities", Ann. Sci. École Norm. Sup. (4) 41 (2008), pp. 1-56.

[Lan10] _ "An Infinite sequence of fixed point free pseudo-ANOSOV homeomorphisms on a genus two surface", Contemporary Mathematics (2010).

[LT10] E. LANNEAU and J-L. ThifFEAUlT - "On the minimum dilatation of pseudo-Anosov homeomorphisms on surfaces of small genus", Annales Fourier 60 (2010).

[Lei04] C. J. LEININGER - "On groups generated by two positive multi-twists: TEICHMÜLlER curves and Lehmer number", Geom. Topo. 8 (2004), pp. 1301-1359.

[Los09] J. LOS - "Infinite sequences of fixed point free pseudo-ANOSOV homeomorphisms.", Erg. Th. Dyn. Sys, to appear (2009).

[MMY05] S. MARMI, P. Moussa and J.-C. YocCOZ - "The cohomological equation for RoTh type interval exchange transformations", Journal of the Amer. Math. Soc. 18 (2005), pp. 823-872.

[MS93] H. MASUR and J. SMILLIE - "Quadratic differentials with prescribed singularities and pseudo-ANOSOV homeomorphisms", Comment. Math. Helv. 68 (1993), pp. 289-307.

[MT02] H. MASUR, S. TABACHNIKOV-"Rational billiards and flat structures", Handbook of dynamical systems, 1A, North-Holland, Amsterdam (2002), pp. 1015-1089.

[McM00] C. T. MCMULLEN - "Polynomial invariants for fibered 3-manifolds and TEICHMÜLLER geodesics for foliation ”, Ann. Sci. École Norm. Sup. 33 (4) (2000), pp. 519-560.

[Pen91] R. Penner - "Bounds on least dilatations", Proc. Amer. Math. Soc. 113 (1991), pp. 443-450.

[HP91] R. PENNER and J. HARER - "Combinatorics of Train Tracks", Annals of Mathematics Studies 125, Princeton University Press (1991).

[Ste09] W. A. SteIN ET AL - "Sage Mathematics Software (Version 4.2.1)", The Sage Development Team, 2009, http://www.sagemath.org

[Rau79] G. RAUZY - "Échanges d'intervalles et transformations induites", Acta Arith. 34 (1979), pp. 315-328. 
[Thu88] W. Thurston - "On the geometry and dynamics of homeomorphisms of surfaces", Bull. A.M.S. 19 (1988), pp. 417-431.

[Tsa09] C. TSAI - "The asymptotic behavior of least pseudo-Anosov dilatations", Geometry \& Topology 13 (2009), pp. 2253-2278.

[Vee82] W. VEeCH - "Gauss measures for transformations on the space of interval exchange maps", Ann. of Math. (2) 115 (1982), no. 1, pp. 201-242.

[Vee89] _ _TEICHMÜLLER curves in modular space, Eisenstein series, and an application to triangular billiards", Inv. Math. 97, (1989) 553-583.

LatP, case cour A, Faculté de Saint Jérôme Avenue Escadrille Normandie-Niemen, 13397 MARSEILLE CEDEX 20

E-mail address: corentin.boissy@latp.univ-mrs.fr

Centre de Physique Théorique (CPT), UMR CNRS 6207

UNIVERSITÉ DU SUD TOULON-VAR AND

FÉdération de ReCherches des Unités de Mathématiques de Marseille

Luminy, Case 907, F-13288 Marseille Cedex 9, France

E-mail address: lanneaulcpt.univ-mrs.fr 